\title{
RECONSTRUCTION \\ AND DETERMINISTIC \\ PREDICTION OF \\ OCEAN WAVES \\ FROM SYNTHETIC \\ RADAR IMAGES
}

ANDREAS PARAMA WIJAYA 


\section{RECONSTRUCTION AND DETERMINISTIC PREDICTION OF OCEAN WAVES FROM SYNTHETIC RADAR IMAGES}




\section{Samenstelling promotiecommissie:}

Voorzitter en secretaris:

prof. dr. P. M. G. Apers

University of Twente

\section{Promotor}

prof. dr. ir. E. W. C. van Groesen University of Twente

\section{Leden}

prof. dr. S. A. van Gils

University of Twente

prof. dr. A. E. P. Veldman

University of Twente

prof. dr. ir. A. W. Heemink

Delft University of Technology

prof. dr. B. Jayawardhana

University of Groningen

dr. G. P. van Vledder

Delft University of Technology

dr. M. Wahab

Indonesian Institute of Sciences (LIPI)

\section{UNIVERSITY OF TWENTE.}

The research presented in this dissertation was carried out at the Applied Analysis group, Departement of Applied Mathematics, Faculty of Electrical Engineering, Mathematics and Computer Science (EEMCS) of the University of Twente, PO Box 217, 7500 AE Enschede, The Netherlands and Laboratorium Matematika Indonesia (LabMath-Indonesia), Jl. Dago Giri no. 99, Warung Caringin, Mekarwangi, Bandung 40391, Indonesia.

This research is motivated by some challenges in the Industrial Research Project entitled "Prediction of waves induced motions and forces in ship, offshore and dredging operations (Promised)", funded by the Dutch Ministry of Economical Affairs, Agentschap NL and co-funded by Delft University of Technology, University of Twente, Maritime Research Institute Netherlands, OceanWaves GMBH, Allseas, Heerema Marine Contractors and IHC Merwede.

Copyright (c) 2017, Andreas Parama Wijaya, Enschede, The Netherlands

Cover: Inez Huang

Printed by Gildeprint, Enschede

ISBN 978-90-365-4362-0

DOI $10.3990 / 1.9789036543620$

https://dx.doi.org/10.3990/1.9789036543620 


\title{
RECONSTRUCTION AND DETERMINISTIC PREDICTION OF OCEAN WAVES FROM SYNTHETIC RADAR IMAGES
}

\author{
DISSERTATION
}

to obtain

the degree of doctor at the University of Twente, on the authority of the rector magnificus, prof. dr. T.T.M. Palstra,

on account of the decision of the graduation committee, to be publicly defended on Thursday $6^{\text {th }}$ of July 2017 at 14:45

\section{Andreas Parama Wijaya}

born on the $4^{\text {th }}$ of December 1986 in Bandar Lampung, Indonesia 
Dit proefschrift is goedgekeurd door de promotor prof. dr. ir. E. W. C. van Groesen 
To my parents 


\section{Summary}

A marine X-band radar is a device that scans the surrounding ocean waves up to distances of some $2 \mathrm{~km}$. A rotating antenna emits electromagnetic beams that are reflected at the water surface and partly received by the antenna and stored as intensity plots every radar rotation time. The coverage of a large observation area makes it possible to detect ships and marine mines at large distances, which was the primary aim of marine radars around the second world war. Since then the contents of the images have been further processed to provide quantitative properties of the surrounding waves, such as directional spectrum, peak period and significant wave height, using the so-called 3DFFT method. Recent research is aimed to get more detailed information from the radar images, the phase-resolved dynamics of the waves. Such information is very much desired for various ocean engineering purposes, such as waves at the coast and near harbors and to reduce downtime of coastal and ocean engineering activities which can only take place during, possibly short times, of low wave conditions, such as helicopter landing, wind mill placements and side-byside loading operations. However, the individual wave prediction from radar images is a difficult task since the images contain at best only much distorted information about the waves. For instance, only part of the waves that are not shadowed by waves closer to the radar, will give a reflection, and the radar backscatter intensity is not directly related to the sea surface elevation.

Successful phase-resolved wave prediction is from very recent times and this dissertation describes our contribution to that. Different from the 3DFFT method, which so far does not seem to be able to detect the waves in a dynamic way, a new method DAES (Dynamic Averaging and Evolution Scenario) has been developed that is based on data assimilation with images that are averaged in a dynamic way. The evolution of a few successive images to the same time brings together information from different parts of shadowed waves and the averaged information improves the quality of the reconstruction of the sea state. Any well reconstructed sea can then be used as initial state for an evolution in time; a wave prediction can be simulated. How long this prediction will be accurate enough depends on the size of the observation area, the velocity of the waves and the quality of the reconstructed sea.

The DAES method has been proved to be successful to reconstruct and predict 
the waves from synthetic images of uni- and bi-modal seas of moderate wave height. The method is recently also tested to reconstruct images from very high seas with nonlinear waves. Then the evolution scenario needs to be adjusted and to evolve the waves nonlinearly the numerical model with pseudo-spectral implementation, called HAWASSI-AB, is used.

In this dissertation, also methods to determine significant wave height and sea surface current from images without any external calibration are presented. These parameters are required as ingredients in the reconstruction method DAES. The significant wave height is needed to scale the reconstructed sea such that the correct amplitude is obtained. The discovery that the significant wave height is related to the intensity of shadowing as function of distance from the radar leads to a successful procedure to obtain the parameter from the images. The sea surface current is needed for a proper propagation model for the seas. Since the DAES method is based on evolving images, an optimization procedure can lead effectively to the correct current values, different from existing other methods. Although all test cases that have been investigated deal with waves above flat bottom, there are preliminary results that indicate that DAES can also be extended to deal with waves above varying bottom close to the coast, a topic for important future research. 


\section{Samenvatting}

Een X-band radar kan de golven in de omgeving van een schip over een afstand van ongeveer $2 \mathrm{~km}$ bestrijken. Een roterende antenne zendt elektromagnetische golven uit die op het water oppervlak worden weerkaatst en gedeeltelijk weer door de antenne worden opgevangen, wat voor elke rotatie een intensiteit beeld oplevert. Het bestrijken van een groot gebied maakt het mogelijk schepen en zeemijnen over grote afstand op te sporen, wat het oorspronkelijke doel van de radar was ten tijde van de tweede wereld oorlog. Sindsdien is de informatie van de beelden verder onderzocht om kwantitatieve gegevens van de omringende zee te verkrijgen, zoals het golf-spectrum, de piekperiode en de significante golfhoogte, met de zogenaamde 3DFFT-methode. Tegenwoordig richt het onderzoek zich op het verkrijgen van verdergaande informatie over individuele golven. Deze kennis is zeer wenselijk omdat veel activiteiten dicht bij de kust of in dieper water slechts plaats kunnen vinden tijdens, misschien korte, perioden van lage golven; voorbeelden zijn het landen van een helikopter op een schip, het plaatsen van windmolens of bij goederen overslag tussen twee schepen. Maar de voorspelling van individuele golven is lastig omdat de radar beelden op z'n best slechts een zeer vertekend beeld van die golven geven. Dat is vooral het gevolg van 'shadowing', het feit dat alleen dat deel van de golf door de radar wordt gezien dat zich niet in de schaduw van een voorafgaande golf bevindt, en vanwege de onduidelijke relatie tussen de beeld-intensiteit en de werkelijke hoogte van de golf.

Het met succes reconstrueren van individuele golven uit radar beelden is pas van zeer recente tijd, en dit proefschrift beschrijft onze bijdragen daaraan. Anders dan de 3DFFT-methode, die tot nu toe daartoe niet in staat lijkt, is een nieuwe methode, DAES, ontwikkeld; deze is gebaseerd op data assimilatie met beelden die in de tijd gemiddeld zijn. Door beelden van verschillende tijd dynamisch naar elkaar te brengen kan informatie van verschillende delen van een golf worden gecombineerd, wat de kwaliteit verhoogt. Een goed gereconstrueerd beeld van de hele zee kan dan gebruikt worden als startpunt voor een voorspelling van de toekomstige zee. De lengte van de voorspelling hangt af van de grootte van het gebied dat de radar bestrijkt, de snelheid van de golven en de kwaliteit van de begin reconstructie.

De DAES methode is succesvol getest met kunstmatig gemaakte radar beelden van enkelvoudige en samengestelde zeeën. Dat geldt voor zeeën met een matige 
golfhoogte, maar ook als de golven extreem hoog zijn. In dat laatste geval moet met niet-lineaire rekening worden gehouden, en wordt voor de voortplanting van golven gebruik gemaakt van het HAWASSI-AB model met een pseudo-spectrale implementatie.

In dit proefschrift worden ook methoden beschreven om de significante golfhoogte en de sterkte van stromingen te bepalen uit alleen de radarbeelden, zonder verdere externe gegevens. Deze grootheden moeten bekend zijn voor het gebruik van DAES. Met de significante golfhoogte kunnen de correcte golfhoogten bepaald worden. Met de ontdekking dat deze grootheid een directe relatie heeft met de mate van shadowing als functie van afstand tot de radar, kan de waarde van de significante golfhoogte direct uit de beelden zelf verkregen worden. De stroomsnelheid is nodig om een goede propagatie van de zee te krijgen. Omdat DAES gebaseerd is op het propageren van beelden, kan een optimalisatie methode de correcte waarde van de stroming leveren, op geheel andere en nauwkeurige manier dan andere methoden. Ofschoon alle testzeeën die tot nu toe beschreven zijn gelden voor het geval de bodem vlak is, zijn er al voorlopige resultaten die erop duiden dat DAES ook uitgebreid kan worden voor golven over variërende bodem, zoals vlak bij de kust, hetgeen een belangrijk toekomstig onderzoeksonderwerp zal zijn. 


\section{Contents}

Summary vii

Samenvatting $\quad$ ix

1 Introduction 1

$1.1 \quad$ Past research on ocean wave inversion from radar images. . . . . . . . 3

1.1.1 Radar for open ocean application . . . . . . . . . . . . . . . 4

1.1.2 Radar for coastal application . . . . . . . . . . . . . . 8

1.2 Contributions in this dissertation . . . . . . . . . . . . . . . . . 9

1.2 .1 The reconstruction of the wave phases . . . . . . . . . . . . . 10

$1.2 .2 \quad H_{s}$ retrieval . . . . . . . . . . . . . . . . . . 11

1.2 .3 Sea surface current determination . . . . . . . . . . . . . . 11

1.2 .4 Nonlinear waves reconstruction . . . . . . . . . . . . . . . . 11

1.3 Outline of the dissertation . . . . . . . . . . . . . . . . . . . . . . . . 12

2 Reconstruction and future prediction of the sea surface from radar observations $\mathbf{1 5}$

2.1 Introduction . . . . . . . . . . . . . . . . . . . . . . . 16

2.2 Synthetic Data . . . . . . . . . . . . . . . . . . . . . . . . . . . . . . . . . . .

2.2 .1 Simplifications . . . . . . . . . . . . . . . . . . . . . . . . . . . . . . . . . . . 18

$2.2 .2 \quad$ Synthetic surface elevations . . . . . . . . . . . . . . . . . 19

$2.2 .3 \quad$ Geometric Images . . . . . . . . . . . . . . . . . . . . . 19

2.3 Dynamic averaging-evolution scenario . . . . . . . . . . . . . 20

2.3 .1 Spatial reconstruction of geometric images . . . . . . . . . . . . 20

2.3 .2 Evolution of a single image . . . . . . . . . . . . . . . . . . 21

$2.3 .3 \quad$ Updates from dynamic averaging . . . . . . . . . . . . . . . . . . . . . . . . . . 22

2.3.4 Evolution and prediction . . . . . . . . . . . . . . . . . . 23

2.4 Case studies . . . . . . . . . . . . . . . . . . . . . 24

2.4 .1 Parameters of the study cases . . . . . . . . . . . . . . 24

2.4 .2 Simulation results . . . . . . . . . . . . . . . . . . . 26

2.5 Discussion of results $\ldots \ldots \ldots \ldots \ldots$ 
2.5 .1 Reconstruction method . . . . . . . . . . . . . . . . 32

2.5 .2 Predictability . . . . . . . . . . . . . . . . . . . . . . . . . . . . . . . . . . . . . 33

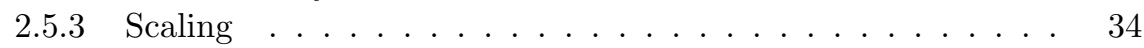

2.5 .4 MED and bimodal sea state . . . . . . . . . . . . . . . 34

2.5.5 Parameter dependence and robustness . . . . . . . . . . . . 34

2.6 Conclusions and remarks . . . . . . . . . . . . . . . . . 35

3 Determination of the significant wave height from shadowing in $\begin{array}{ll}\text { synthetic radar images } & 37\end{array}$

3.1 Introduction . . . . . . . . . . . . . . . . . . . . . . . . . . . . . . . . . . . .

3.2 Synthetic Data . . . . . . . . . . . . . . . . . . . . . . . . . . . . . . . . . . .

3.2.1 Synthetic sea . . . . . . . . . . . . . . . . . . . . . . . . . . . . . 40

3.2.2 Synthetic radar images. . . . . . . . . . . . . . . . . . . . . . . . . . . . . . . . . 4

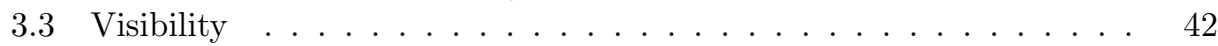

3.3.1 Dimensionless variables . . . . . . . . . . . . . . . . . . . . 42

3.3.2 Visibility of long crested harmonic waves . . . . . . . . . . . . . . . 43

3.3.3 Visibility of irregular waves . . . . . . . . . . . . . . . . . . . . . 44

3.4 Hs estimation method . . . . . . . . . . . . . . . . . . . 46

3.4.1 Minimal Visibility Direction (MiViDi) . . . . . . . . . . . . . . 48

3.4 .2 Design of database using model spectrum . . . . . . . . . . . . 48

3.4 .3 Design of database using an observed spectrum . . . . . . . . 48

3.4.4 Curve-fitting to estimate $H_{s} \ldots \ldots \ldots$. . . . . . . . . . . . . . . . . . . 49

3.5 Case Studies . . . . . . . . . . . . . . . . . . . 50

3.5.1 Preparation for the visibility . . . . . . . . . . . . . . . 50

3.5.2 Preparation of the visibility database. . . . . . . . . . . . . . . 52

3.5.3 Estimation of $H_{s} \ldots \ldots \ldots \ldots$. . . . . . . . . . . . . . . . . . . . . . . . . . 54

3.6 Conclusion and remarks . . . . . . . . . . . . . . . . 57

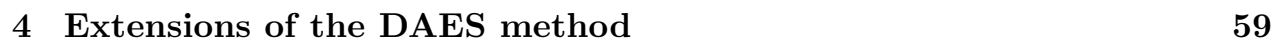

4.1 Sea Surface Current Detection _ . . . . . . . . . . . . . . . . 59

$4.1 .1 \quad$ Introduction $\ldots \ldots \ldots \ldots$. . . . . . . . . . . . . . . . . . . . . . . . . . . . . 60

4.1 .2 Synthetic data . . . . . . . . . . . . . . . . . . . . . . . 61

$4.1 .3 \quad$ Surface current detection . . . . . . . . . . . . . . . . . 62

4.1 .4 Study cases and results . . . . . . . . . . . . . . . . . . . . . . . . . . . . 63

4.1 .5 Conclusions . . . . . . . . . . . . . . . . . . . 64

4.2 Reconstruction and prediction of nonlinear waves . . . . . . . . . . 66

$4.2 .1 \quad$ Parameters of synthetic Draupner sea . . . . . . . . . . . 68

$4.2 .2 \quad$ Dynamic averaging and evolution of synthetic images . . . . 70

4.2 .3 Prediction Results . . . . . . . . . . . . . . . . . . . . . . . . . 72

4.2 .4 Discussion . . . . . . . . . . . . . . . . . . . . . . 77

$\begin{array}{lll}5 & \text { Outlook } & 79\end{array}$

\begin{tabular}{lr}
\hline Bibliography & 81
\end{tabular}

\begin{tabular}{|lr}
\hline Acknowledgments & 87
\end{tabular} 
\begin{tabular}{lr}
\hline About the author & 89
\end{tabular} 



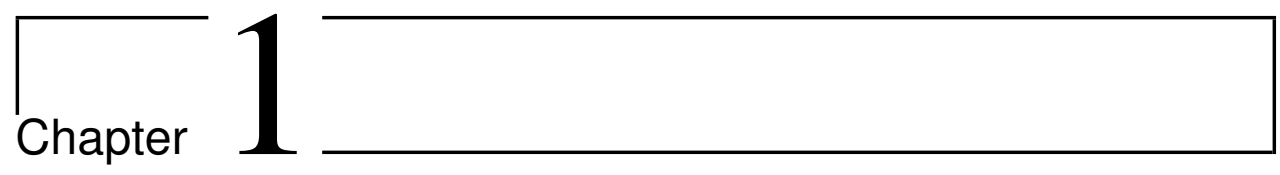

\section{Introduction}

Marine radars have been used extensively in the last decades as a tool to measure some, mainly quantitative aspects, of ocean waves. The capability of marine radars to cover a large observation area is a great potential to be explored. Some wave phenomena could, in principle, be directly observed, such as refraction, reflection, and diffraction, which are impossible to observe by a buoy, the common in situ measurement device. For coastal application, wave information can help to improve the optimal design of coastal structures and to increase safety in ship navigation near the coast. Motivated by the disastrous 2004 Indian ocean tsunami, a study to investigate the capability of a radar to detect tsunamis was carried out in Lipa et al. 2006. The 2004 tsunami is the deadliest tsunami in the recorded history with a death toll estimated at around 220.000. The destructive effect can be seen in the Fig 1.1 that shows the comparison before and after the tsunami hit in the coastal area of Aceh province of Indonesia. The massive tsunami which was generated by an earthquake of moment magnitude 9.0-9.3 caused the death of more than 200.000
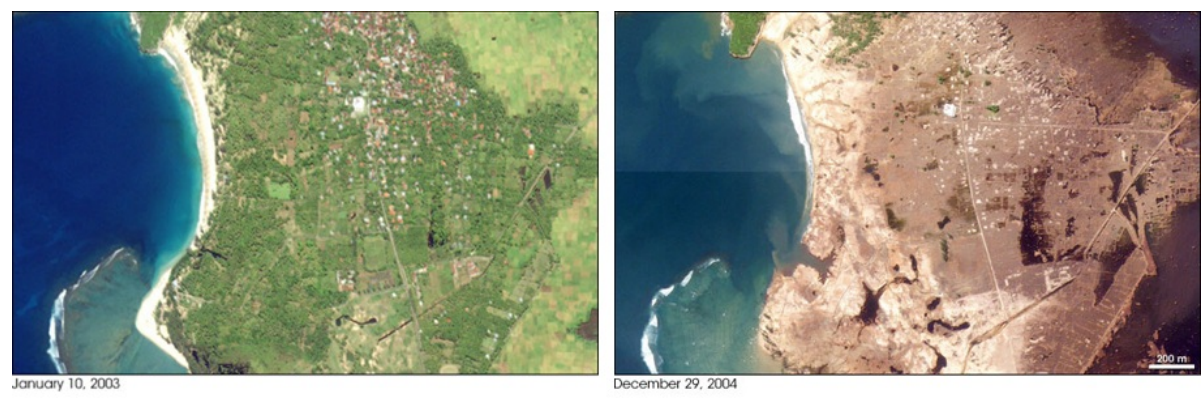

Figure 1.1: The town of Lhoknga in Aceh Province of Indonesia on January 10, 2003 before the 2004 Indian Ocean tsunami and the aftermath of the disaster. Copyright Centre for Remote Imaging, Sensing and Processing, National University of Singapore and Space Imaging. 
people, a number that may have been less if an early tsunami warning system would have been in place. In early 2005 after the tragedy of the Indian Ocean tsunami, a tsunami warning system was built by using buoys. Indonesia itself has 22 buoys that are deployed from the West until the East Indonesian ocean. Unfortunately, all the buoys, which costed millions of US dollar are broken due to a lack of maintenance. In recent years, some studies have been executed to prepare radars as a tsunami early warning system, e.g. Dzvonkovskaya et al. [2011], Grilli et al. 2016.

Apart from the tsunami application, the main use of radars in measuring the ocean waves is to support ocean engineering activities. To that end, accurate and real-time wave forecasting methods based on radar images are more and more in great demand. Providing the predicted time window of low sea conditions a few minutes ahead will support offshore operations, such as helicopter landing, wind mill installation, and side-by-side loading operation. For such sensitive operations the predicted wave information can be very beneficial to reduce the risk of operation failures or damage structures and to reduce 'down time', the periods of rather low waves in a relatively high sea that without a priori knowledge would not have been used for such operations.

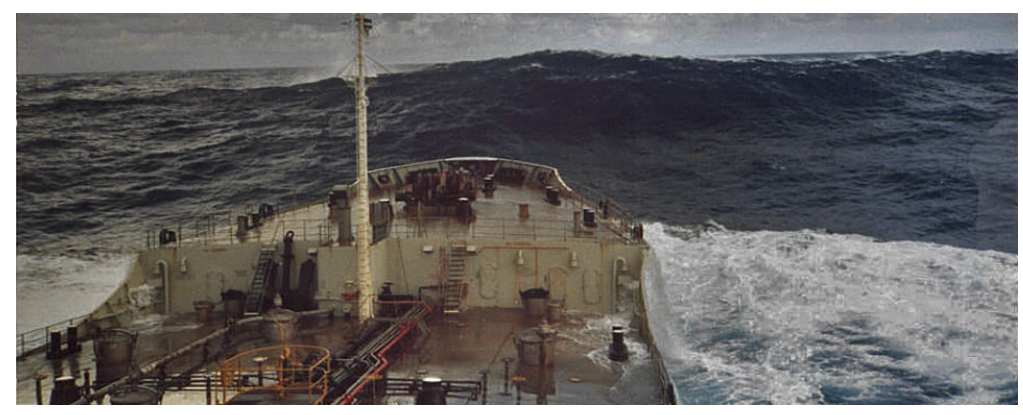

Figure 1.2: A rogue wave estimated at 18.3 meters (60 feet) in the Gulf Stream off of Charleston, S.C. At the time, surface winds were light at 15 knots. The wave was moving away from the ship after crashing into it moments before this photo was captured. http://oceanservice.noaa.gov/facts/roguewaves.html

Nowadays, most ships are equipped with marine radars as navigational aid and to prevent collisions. However, many ship accidents are caused by high waves. The socalled rogue waves (sometimes called freak, extreme, or killer waves), defined as the waves that are greater than twice the significant wave height, can occur unexpectedly without warning from any direction. A photograph in Fig. 1.2 shows a rogue wave of about 18.3 meters in the Gulf Stream off of Charleston, S.C. Apart from the rogue waves generated by nature, other "rogue" waves come from another source, namely ships. A moving ship generates waves, called ship wake, that could be very harmful for another nearby ship, especially when the visibility is low. With a system that can process radar images to yield accurate and real-time wave prediction, a sailor will be able to navigate his ship in a safer sailing path, avoiding the possible high waves that could endanger the ship.

However, wave forecasting from radar images is a challenging task. How far 


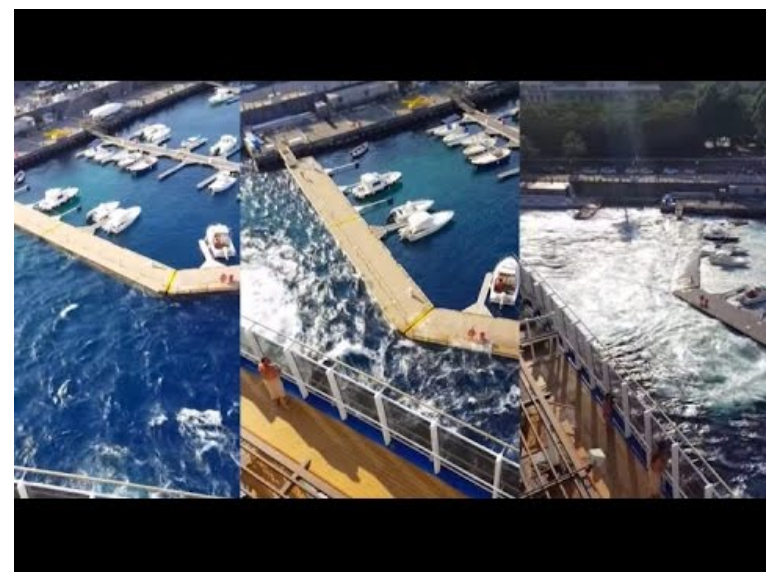

Figure 1.3: The Carnival Vista cruise ship generated ship wake that destroyed a marina in Sicily, Italy. http://www.huffingtonpost.com/entry/cruise-ship-destroysmarina_us_57d5810be4b03d2d459aec04

ahead in time the wave elevation can be predicted at the radar location depends fundamentally on the size of the observation area and the wave (group, phase) velocity. Since radar images do not represent the observed waves perfectly, the reconstruction process from images into the sea surface elevations also affects the quality of the prediction as well. Moreover, a fast and accurate numerical wave model is required to evolve and predict the sea surface elevation in future time.

The wave inversion from radar images to deterministic phase resolved waves has attracted many scientists and has been in great demand from offshore companies. Many methods have been developed regarding this topic and will be summarized in the first subsection. The contribution and the outline of this dissertation are then given in the second and the third subsection respectively.

\subsection{Past research on ocean wave inversion from radar images}

Radars are in use extensively since the second world war with the original aim to detect ships, naval mines and aircrafts. After the war, the applications were further expanded as navigational aid, especially for times when the visibility is poor. The radar technique then evolved to be used as a tool for the description of the sea state (e.g. Crombie 1955, Munk and Nierenberg 1969, Barrick [1972]). It was concluded that the most important mechanism in the interaction between the electromagnetic beams radiated from the radar and the ocean waves is Bragg scattering Valenzuela, 1978; for a beam at an incidence angle $\theta$, the waves with wavenumber $2 k \sin (\theta)$ (the Bragg resonance condition) contributes significantly to the radar backscatter. There are basically two types of radars used for scanning the ocean waves, incoherent radars that measure the radar cross section (the quantity of how detectable the sea 
surface is with the radar) in a surrounding ring shaped area between $300 \mathrm{~m}$ and $2000 \mathrm{~m}$, and coherent Doppler radars that measure the horizontal fluid velocity till distances of some $10 \mathrm{~km}$. For typical marine incoherent radars operated at the Xband frequency $(9.5 \mathrm{GHz})$ with wavelength around $2-4 \mathrm{~cm}$, the small ripples induced by the wind on the sea surface cause a radar return. These return signals received at the radar will produce a radar backscatter plot every rotation of the radar, which is typically between 1 and 2 seconds. This radar backscatter has no direct relation with the (significant) wave height of the surrounding waves. The longer waves are visible in radar images due to modulations of the radar cross section. There are four modulations in the radar mechanism, i.e. hydrodynamic, tilt, shadowing, and wind. Hydrodynamic modulation describes the distribution of the short waves with respect to the longer waves Alpers et al. 1981]; tilt modulation measures the projection of reflected radar rays normal to the surface elevation; shadowing is the effect that for low grazing angles waves further away will be partly blocked by the waves closer to the radar; wind modulation determines the strength of the reflected signal due to the wind (speed and direction) in creating the roughness on the sea surface.

This section summarizes two application areas for which the radar (mostly incoherent X-band) is used for waves observation. The first subsection describes the past work of the use of radars for the open ocean application. For this case a radar is usually mounted on a ship (stationary or moving) or on a fixed offshore platform. A constant water depth can be assumed and the waves are nearly homogeneous. The second subsection summarizes the study of the radar applied in coastal areas which is more complicated in deriving either the wave properties or the phase resolved waves. This is because of the non-homogeneous properties in the coastal areas; for instance varying bathymetry, nonlinear wave effects, and wave breaking.

\subsubsection{Radar for open ocean application}

The first attempt in analyzing X-band radar images was mainly to retrieve the normalized spectrum. In Hoogeboom and Rosenthal 1982 and Ziemer and Rosenthal 1983, a 2D spectrum was obtained by applying the 2D Fourier transform to the digitized radar image and was shown to be similar to the spectrum obtained from buoy measurements. However, the wave direction can not be resolved since the $2 \mathrm{D}$ Fourier transform yields $180^{\circ}$ directional ambiguity. A simple numerical scheme has been proposed in Atanassov et al. [1985] to remove the directional ambiguity by using two consecutive images and the dispersion relation. In Young et al. 1985, a $3 \mathrm{DFFT}$ method was used to derive a 3D spectrum. To obtain the unambiguous directional spectrum, the $3 \mathrm{D}$ spectrum was integrated with respect to the positive frequencies. This 3DFFT method then became the common method to derive several wave properties from radar images. In Borge et al. 2004, they observed a difference between the image spectrum obtained by 3DFFT method and the corresponding wave spectrum from a buoy measurement. The radar imaging mechanisms, e.g. shadowing and tilt modulation, are responsible for the difference. To retrieve the wave spectrum from radar images, the use of an empirical Modulation Transfer 
Function (MTF) was proposed. It was calculated as

$$
|M T F(k)|^{2}=\frac{F_{r}(k)}{F_{i s}(k)}
$$

where $F_{r}(k)$ is the $1 \mathrm{D}$ wavenumber spectrum from the derived 3D image spectrum and $F_{i s}(k)$ is the wavenumber spectrum obtained from a buoy measurement. Based on the numerical simulations and the measuring campaign close to the Spanish northern coast on 14 February 1995, it was concluded that the MTF is proportional to $k^{1.2}$.

Although the aforementioned methods successfully estimated the wave spectrum which have a comparable shape to the resulted spectrum from buoy measurements, the wave energy had not been resolved yet. Some researches have been carried out to estimate the significant wave height, which is proportional to the wave energy. Roughly speaking, two different methods have been used in the past to achieve that aim; one using the reconstruction spectrum, and one using the spatial dependence of the shadowing phenomenon. The most commonly used method in the spectrumapproach is to estimate $H_{s}$ from the so-called signal-to-noise ratios (SNR). It was introduced by Alpers and Hasselmann [1982] for synthetic aperture radar (SAR). The SNR was used to estimate the sea spectrum such that $H_{s}$ was calculated as four times the square root of the estimated spectrum area Plant and Zurk, 1997. For X-band radar images, the 3DFFT method was used in Borge et al. 1999 to calculate the SNR as

$$
S N R=\frac{\int_{\mathbf{k}, \omega} F(\mathbf{k}, \omega) d \mathbf{k} d \omega}{\int_{\mathbf{k}, \omega} F_{b g n}(\mathbf{k}, \omega) d \mathbf{k} d \omega}
$$

where $F(\mathbf{k}, \omega)$ is the band-pass filtered 3D spectrum by the exact linear dispersion and $F_{b g n}(\mathbf{k}, \omega)$ is the spectrum of the components outside the dispersion relation. In contrast to Plant and Zurk 1997, $H_{s}$ was taken to be linearly related to the square root of the SNR with two free parameters which were calibrated from insitu measurements. This method was applied on the radar data provided by radar system WaMoS II which has been developed at the German GKSS research centre Hilmer and Thornhill, 2015.

The other approach used the distribution of shadowed areas that result because of geometrical shadowing, which is the effect that waves closer to radar can block the ray so that waves further away become invisible. In this respect, it should be remarked that in Plant and Farquharson 2012a it was argued that the geometric shadowing does not play a role in the radar mechanism; in contrast, partial shadowing is claimed to be the effect that appears in the images. The given explanation is that the diffraction of the electromagnetic signal causes a backscatter signal from the surface elevation that occupies the geometrical shadowed areas. However, the partial shadowing depends on the type of the polarization from the radar, and the difference with the geometrical shadowing may be very small.

Concerning the geometric shadowing, a statistical concept based on the proportion of the visible ('islands') and the invisible ('troughs') part of the waves was introduced by Wetzel [1990]. The probability of illumination $P_{0}$ was defined and related to $H_{s}$. In Buckley and Aler 1998a it was shown that the estimation of $H_{s}$, 
using a constant $P_{0}$ that was calibrated from in-situ measurements, was only accurate for certain wave conditions, for instance when the ration of radar height and the wave height was high. An improvement was obtained by varying $P_{0}$ as shown in Buckley and Aler 1998b. A method without using any reference measurement, described in Gangeskar [2014], estimated $H_{s}$ from the RMS of the surface slope which is related to the shadowing effect. The relation is found from the best fit of the shadowing ratio, the proportion of the invisible points as a function of grazing angle, with the so-called Smith's function [Smith, 1967]. The results compared to measurement with a correlation of $87 \%$.

In Wijaya and van Groesen 2014], a method to estimate $H_{s}$ for long-crested waves based on the geometrical shadowing has been reported. The basic idea of the method is that the amount of shadowing is related to $H_{s}$. Formulations to measure the shadowing level were derived earlier by Wagner [1966], Smith 1967], and compared to experiments described in Brokelman and Hagfors 1966]. These formulations assumed that the joint probability density of heights and slope was uncorrelated. It was verified later by Bourlier et al. [2000] that the correlated joint probability density of heights and slope performed better than the uncorrelated one compared with the shadowing function that was determined numerically by generating the surface Brokelman and Hagfors, 1966.

Another sea parameter that can be derived from radar images is the sea surface current. The presence of the current changes the wave velocity; the waves move faster if the current direction is in the wave direction, otherwise the waves will move slower. The frequency of the waves induced by a current $\mathbf{U}$ is called the encounter and modeled as

$$
\Omega_{e n}(\mathbf{k})=\Omega_{0}(\mathbf{k})+\mathbf{U} \cdot \mathbf{k}
$$

where $\Omega_{0}=\sqrt{g|\mathbf{k}| \tanh (|\mathbf{k}| d)}$ is the intrinsic frequency, $d$ is the water depth and $\mathbf{k}=\left(k_{x}, k_{y}\right)$ is the wavenumber vector. The comparison of the intrinsic and the encounter frequency is shown in Fig 1.4. In this example a current directed to the North is added which can be recognized in the right plot of Fig 1.4 the higher frequencies occur at the positive wavenumbers $k_{y}$.
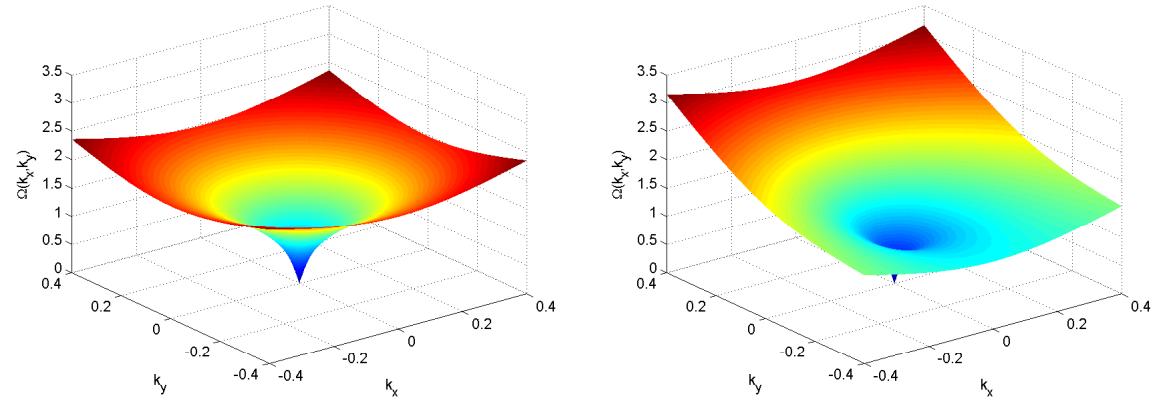

Figure 1.4: The intrinsic dispersion relation at the left and the encounter dispersion is shown at the right.

Young et al. 1985 concluded that any deviation from the intrinsic dispersion 
relationship is due to a current induced Doppler shift of the wave frequency. The surface current was then estimated by curve fitting between the derived frequency from the 3DFFT method and the theoretical model frequency in Eq. 1.3. The method was improved in Senet et al. 2001] where nonlinear spectral structures were considered to increase the number of regression components. Furthermore, temporal aliasing due to the slow antenna radar rotation was applied to improve the accuracy of the curve fitting. The use of a 3D spectrum as a weighted function in the curve fitting technique was proposed in Gangeskar 2002]. A different technique to derive the surface current was introduced in Serafino et al. [2010]. The maximum of the socalled Normalized Scalar Product (NSP) determined an estimated current velocity. The NSP was defined as

$$
N S P(\mathbf{U})=\frac{\left\langle\left|F_{I}(\mathbf{k}, \omega)\right|, G(\mathbf{k}, \omega, \mathbf{U})\right\rangle}{\sqrt{P_{F} \cdot P_{G}}}
$$

Here, $F_{I}$ is a filtered 3D image spectrum, $G$ is the band pass filter of the encounter frequency defined in Eq. 1.5, $P_{F}$ and $P_{G}$ are the power associated to $|F|$ and $G$ respectively.

$$
G(\mathbf{k}, \omega, \mathbf{U})=\left\{\begin{array}{l}
1, \text { if }\left|\Omega_{0}(\mathbf{k})+\mathbf{U} \cdot \mathbf{k}-\Omega_{e n}(\mathbf{k})\right|<\Delta \omega / 2 \\
0, \text { otherwise }
\end{array}\right.
$$

The NSP method was able to detect relatively high speed currents, but required a long computation time. In Huang et al. [2012], the method has been improved for both the computational efficiency and the precision by narrowing the variable search ranges iteratively. Instead of the 3DFFT approach, 2DFFT methods (e.g. Alford et al. 2014 and Abileah and Trizna 2010]) can be used as an alternative method to derive the surface currents. Although the 2DFFT approach may suffer from the presence of noise, it requires shorter time series than 3DFFT which is more suitable in real-time applications.

Although the 3DFFT method is quite successful to determine the characteristic parameters of the sea, efforts to obtain phase resolved information about the surrounding wave field were not successful Naaijen and Blondel 2012]. In Borge et al. 2004, after some filtering procedures on the calculated 3D amplitude image spectrum, the Fourier components of the wave amplitude were obtained by applying the inverse MTF (Eq1.1) to the filtered amplitude spectrum. Inverting the Fourier amplitude components with the phase image spectra yielded the sea surface elevation. The retrieved wave spectrum as well as the wave height probability distributions from the wave elevation maps had a good agreement with the buoy measurement. However, the comparison of the individual phase resolved waves was not given. Another approach based on variational data assimilation scheme was proposed in Aragh and Nwogu 2008 and Aragh et al. 2008] to find optimal wave profiles that minimize the difference between images and a wave model prediction led to an approximation of the multi-directional spectrum over part of the frequency band.

Reconstruction and prediction of phase resolved waves at the ship position from radar images have become successful in the last few years. Previously, an empirical method in Dankert and Rosenthal 2004] required the radar images to be free of 
shadowing effects, which can only be achieved when the radar is mounted very height relative to the significant wave height. A forecast system, called Computer Aided Ship Handling (CASH) Clauss et al. 2012, recovers the surface elevations from the Fourier components of the radar images. In Alford et al. [2014] a combination of Doppler and backscatter data was used to estimate the surface elevation by finding the Fourier components that minimize the error between the Polar Fourier Transform of the images and a wave model; retrieved time signals were found to be in good agreement with buoy data after some filtering.

\subsubsection{Radar for coastal application}

For coastal applications, the analysis of radar images is mainly to derive the bathymetry (and the sea surface current). Based on linear wave theory, a bathymetric inversion equation has been derived in Bell 1998 and expressed as

$$
d=\frac{T}{2 \pi} C \tanh ^{-1}\left(\frac{2 \pi C}{g T}\right)
$$

where $C$ is the phase velocity and $T$ is the (peak) wave period. The inversion was solved by finding these two quantities $C$ and $T$. The velocity was obtained by calculating the auto-correlation between consecutive images to detect the wave motion on small areas in the radar images. The period $T$ was retrieved from buoy data although it was found later in Bell 1999 that the peak spectrum from the buoy was different from the one derived by the radar.

A similar method as in Bell [1998 was presented in Hessner et al. [1999]. A bathymetric function that depends on the frequency $\omega$ and the wavenumber $k$ was derived from the exact dispersion relation. As the waves from deeper area reach the beach area, wave shoaling occurs. The frequency does not change so much in shoaling events Holthuijsen, 2007, hence only the local wavenumber $k$ need to be found to determine the water depth. The local wavenumber was obtained by calculating the local phase gradient under the assumption of a monochromatic wave. The limitation of the method in Bell 1998 and Hessner et al. 1999 is that the sea surface current and the effect of nonlinearity were not considered. Bell [2008] improved the method that take those properties into account.

The extension of the NSP method to determine the water depth was presented in Serafino et al. 2010 for long-crested case. The method under the so-called Remocean processing system was tested on real radar data obtained from field experiments in Giglio Island port, Italy Ludeno et al. 2014 and in Salerno Harbour, Italy Ludeno et al. 2015. A systematic error in the result was found which was supposedly from the assumption of the linear wave theory in the method leading to an overestimation of the water depth, particularly in shallow water. Based on the estimated water depth and sea surface current, the sea surface elevations were calculated by using MTF defined in Borge et al. 2004.

WaMoS II has been used to monitor and measure waves in the coastal area. An experiment in the island of Sylt, Germany and at the Port Phillip Bay, Australia was carried out in Reichert and Lund 2007. The wave phenomena such as refraction, shoaling, and dissipation were observed in the radar images. In a recent publication 
Punzo et al. 2016], the Remocean system was used for another experiment in Bagnara Calabra Italy not only to determine surface current and bathymetry, but also to detect rip currents, see Fig. 1.5 .

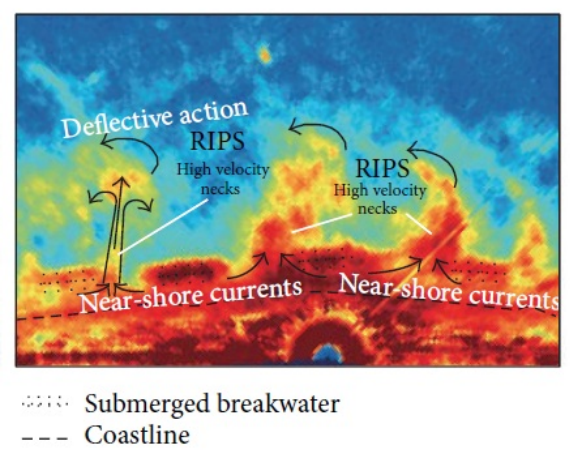

Figure 1.5: A subimage radar shows rip currents at a coast in Bagnara Calabra Punzo et al., 2016.

Radars in coastal areas can be used as an early tsunami warning system. Different than the descriptions above that use X-band radar, HF radar is employed to be useful to detect tsunami. This type of radar is generally used to measure sea surface current up to $200 \mathrm{~km}$ away with resolution ranging from $500 \mathrm{~m}$ to $6 \mathrm{~km}$. The surface current is detected by measuring the Doppler shift analyzed in the received sea echo signal Barrick et al. 1978. Its capability to measure surface current in the order of accuracy of $10 \mathrm{~cm} / \mathrm{s}$ leads to the extension to detect tsunamis by measuring their orbital wave velocity as they approach the coast Barrick, 1979. The method is then tested with a numerical simulation in Lipa et al. [2006]. The limitation of this approach is that the tsunami current should be at least $0.1 \mathrm{~m} / \mathrm{s}$ to make this approach works. An improvement has been made to overcome this limitation in Grilli et al. 2016].

\subsection{Contributions in this dissertation}

This dissertation presents a new approach for reconstructing ocean waves from (synthetic) radar images. The images are set up by locating a radar at the center that scans the surrounding waves; around the radar no information is available. The shadowing distort the image quite severely especially at low grazing angle where almost all wave troughs have disappeared, and can be regarded as a representative of poor images. There are four aspects of the waves reconstruction from images that are discussed in this dissertation:

1. resolving the phase of waves,

2. retrieving the significant wave height,

3. detecting the sea surface current, 
4. reconstructing non-linear waves.

The following subsections give an overview of what has been done regarding to these aspects.

\subsubsection{The reconstruction of the wave phases}

The first stage to reconstruct a sea image $I_{n}$ (at time $n \Delta t$ ) is by making the spatial average of the radar image to be zero. Due to shadowing the significant wave height of the image is not the same as the actual $H_{s}$. With a scaling factor $C$ to obtain the correct $H_{s}$, the reconstructed image in Cartesian coordinates with the correct significant wave height is obtained as

$$
R_{n}(\mathbf{x})=C\left(I_{n}(\mathbf{x})-\operatorname{mean}\left(I_{n}\right)\right)
$$

The reconstruction procedure as described above does not yet recover the shadowed area. To further improve the image, an averaging procedure in physical space is applied. This procedure involves three successive reconstructed images $R_{n}$ and one updated image from previous averaging.

We describe the averaging evolution scenario at a certain averaging time $t_{0}$, which is a multiple of $3 d t$. At that time the available information consists of the reconstructed images $R_{0}, R_{-1}, R_{-2}$ at time $t_{0}, t_{0}-d t, t_{0}-2 d t$ respectively and an updated image $U_{-1}$ obtained from previous averaging at time $t_{0}-3 d t$. It can be expected that an averaging procedure will reduce the inaccuracy that appears in each reconstructed image $R_{k}$, provided that this averaging is done dynamically to compensate for the fact that the images are available at different times. Therefore, taking also the evolution of the previous update into account, the images $R_{-1}, R_{-2}, U_{-1}$ are evolved over $d t, 2 d t, 3 d t$ respectively. These evolution images, just as $R_{0}$, then represent approximations of the sea surface at time $t_{0}$. Each approximation may contain different errors due to different inaccuracies of shadowed waves at each image. Therefore, the averaged information will enhance the quality of the approximation of the sea state. The evolution of $U_{-1}$ will contain information of the elevation in the area near the radar where the $R_{n}$ are vanishing. With some weight factors the updated image at the averaging time $t_{0}$ is taken as

$$
\begin{aligned}
U_{0}(\mathbf{x}) & =\left(\frac{1}{6}\left(R_{0}+\mathcal{E}^{1}\left(R_{-1}\right)+\mathcal{E}^{2}\left(R_{-2}\right)\right)+\frac{1}{2} \mathcal{E}^{3}\left(U_{-1}\right)\right)\left(1-\chi_{\text {rad }}\right) \\
& +\mathcal{E}^{3}\left(U_{-1}\right) \chi_{\text {rad }}
\end{aligned}
$$

Here, $\mathcal{E}^{n}(R)=\mathcal{E}(R, n \cdot d t)$ denotes the linear evolution of image $R$ over time $n \cdot d t$ and $\chi_{\text {rad }}$ is the characteristic function denoting the near radar area: $\chi_{\text {rad }}=1$ for area of inner radius $r_{i n}$ around the radar and $\chi_{\text {rad }}=0$ for the remaining area.

By using any updated image $U_{0}$ at any time $t_{0}$, a prediction can be carried out without using any information of images $R_{n}$ later than $t_{0}$. The prediction for a future time $\tau \in[0, \Pi]$, where $\Pi$ is the prediction horizon, is then defined as

$$
P\left(U_{0}, \tau\right)=\varepsilon(R, \tau) \text { for } \tau \in[0, \Pi]
$$




\subsection{2 $\quad H_{s}$ retrieval}

To determine $H_{s}$ from shadowed images, the basic idea of the method is that the visibility in sequences of images can be used to determine the significant wave height. To measure the severity of shadowing, a visibility function is defined as the probability that the surface elevation is visible at a certain position. Hence, the visibility calculated from $M$ images can be written as

$$
v i s(r, \theta)=\frac{1}{M} \sum_{i=1}^{M} \chi_{i}(r, \theta)
$$

Here, $\chi_{i}(r, \theta)$ is the characteristic function to indicate the visible points at distance $r$ on angle direction $\theta$ in image- $i$. The visibility function depends on the radar height $H_{r}$, and the sea parameters such as the peak wavelength $\lambda_{p}$, the significant wave height $H_{s}$ and the shape of the sea spectrum. In fact, only two dimensionless quantities in the horizontal and vertical direction, $\rho=\frac{r}{\lambda_{p}}$ and $h=\frac{H_{r}}{H_{s}}$ respectively, determine the visibility. Knowing the water depth and the normalized sea spectrum will make $H_{s}$ the only unknown parameter that determines the visibility. This is used to design a visibility database for various discrete values of $h$ based on an estimated sea spectrum. Then, the visibility as obtained from radar images can be fitted to a visibility curve in the database which then determines the significant wave height of the observed sea. The method consists of the following main steps: the estimation of the normalized sea spectrum, the construction of the visibility database, and the curve fitting to estimate $H_{s}$, which will be discussed in detail in Chapter 3 .

\subsubsection{Sea surface current determination}

Most used methods of sea surface current detection are based on the comparison of the dispersion-current model and the frequencies derived from images. In this dissertation, a different approach which employs the DAES method is presented. The basic idea is that the surface current can be determined by comparing different physical images. Since the images contain many inaccuracies, the comparison is now between the reconstructed image from DAES with the reference image. The surface current is then determined by solving

$$
\min _{\mathbf{U}}\left\|D A E S\left(\left\{R_{0}, R_{1} \ldots, R_{n}\right\}, \mathbf{U}\right)-R_{n}\right\|_{D}^{2}
$$

Here, $D A E S\left(\left\{R_{0}, \ldots, R_{n}\right\}, \mathbf{U}\right)$ is the result of the reconstruction DAES method from the image set $\left\{R_{0}, \ldots, R_{n}\right\}$ with the linear evolution that takes the current velocity $\mathbf{U}$ into account. The difference between the reconstructed image and the reference image $R_{n}$ is computed on a sub-domain $D$ which will be chosen as a rectangular area heading the main wave direction.

\subsubsection{Nonlinear waves reconstruction}

The effect of nonlinearity on the visibility as defined above are substantial. This can be expected since the nonlinear effect increase the crest heights (and reduce trough 
depths) which leads to the lower visibility. Such nonlinear effects imply that the evolution scenario in Eq. 1.8 has to be adjusted with a nonlinear evolution. Since the nonlinear effect requires a sufficient time to grow, the nonlinear evolution applies only for the updated image $U$. An updated image with the adjusted DAES method is then expressed as

$$
\begin{aligned}
U_{0}(\mathbf{x}) & =\left(\frac{1}{6}\left(R_{0}+\mathcal{E}^{1}\left(R_{-1}\right)+\mathcal{E}^{2}\left(R_{-2}\right)\right)+\frac{1}{2} \mathcal{E}_{n l}^{3}\left(U_{-1}\right)\right)\left(1-\chi_{\text {rad }}\right) \\
& +\mathcal{E}_{n l}^{3}\left(U_{-1}\right) \chi_{r a d}
\end{aligned}
$$

where $\mathcal{E}_{n l}($.$) denotes the nonlinear evolution operator for which the Analytic Bous-$ sinesq model of HAWASSI software LabMath-Indonesia 2015 has been used.

\subsection{Outline of the dissertation}

This dissertation consists of an introduction, three main chapters, and an outlook; the first two main chapters are based on two journal papers whereas the last main chapter is a combination of a conference paper and a part of a submitted journal paper. The organization of this dissertation is as follows. Chapter 2 describes the reconstruction method DAES to obtain phase-resolved wave from the radar images which are synthesized from uni- and bi-modal seas by taking into account only the shadowing. From a reconstructed image, a prediction is carried out for 2-3 minutes ahead depending of the size of the observation domain and the wave (group and phase) velocity. In Chapter 3 the method to retrieve significant wave height based on the analysis of shadowing areas is discussed. Chapter 4 consists of two studies: the detection of sea surface current from tilt-shadowed images and the investigation of the method in Chapter 2 to deal with a rogue Draupner-like sea. In Chapter 5 an outlook presents a preliminary result of the DAES method to overcome more complex sea for long-crested sea. A brief summary of the three main chapters is given as follows.

\section{Chapter 2: Reconstruction and future prediction of the sea surface from radar observations}

This chapter is a published paper of DAES method Wijaya et al. 2015. The construction of synthetic linear seas and synthetic images, which take only shadowing into account, is described in Section 2.2. The framework of the DAES method is presented in Section 2.3 that includes the details of the spatial reconstruction of an image, the linear evolution of a single image, the updates from dynamic averaging, and the prediction from a reconstructed image. Two case studies are performed, uni- and bi-modal sea, in Section 2.4 and the results are discussed in Section 2.5. This chapter ends with conclusions and remarks.

Chapter 3: Determination of the significant wave height from shadowing in synthetic radar images

This chapter has been published as Wijaya and van Groesen 2016] which proposes a method to determine the significant wave height based on shadowing from radar 
images. The method is tested on synthetic data given in Section 3.2. The measure of shadowing is defined by the visibility discussed in Section 3.3 . The procedure to determine the significant wave height from the visibility is given in Section 3.4. The results of the same case studies as in Section 2.4 are presented in Section 3.5. This chapter is closed with conclusions and remarks.

\section{Chapter 4: Extensions of the DAES method}

This chapter describes two extensions of the DAES method: to detect sea surface current (published as a conference paper Wijaya 2017]) in Section 4.1, and to reconstruct nonlinear waves (a part of a submitted paper van Groesen et al. 2017]) in Section 4.2. The method of detecting the sea surface current is applied on images of a synthesized linear sea with additional surface currents. The images are created by taking the shadowing and tilt modulation into account. For the nonlinear waves case, the sea is simulated by nonlinear $\mathrm{AB}$ code with wave influx based on a $2 \mathrm{D}$ Draupner spectrum. 



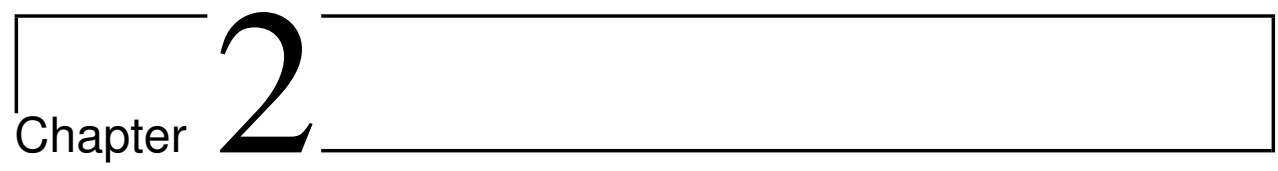

\section{Reconstruction and future prediction of the sea surface from radar observations 1}

\section{Abstract}

For advanced offshore engineering applications the prediction with available nautical $\mathrm{X}$-band radars of phase-resolved incoming waves is very much desired. At present, such radars are already used to detect averaged characteristics of waves, such as the peak period, significant wave height, wave directions and currents. A deterministic prediction of individual waves in an area near the radar from remotely sensed spatial sea states needs a complete simulation scenario such as will be proposed here and illustrated for synthetic sea states and geometrically shadowed images as synthetic radar images. The slightly adjusted shadowed images are used in a dynamic averaging scenario as assimilation data for the ongoing dynamic simulation that evolves the waves towards the near-radar area where no information from the radar is available. The dynamic averaging and evolution scenario is rather robust, very efficient and produces qualitatively and quantitatively good results. For study cases of wind waves and multi-modal wind-swell seas, with a radar height of 5 times the significant wave height, the correlation between the simulated and the actual sea is found to be at least $90 \%$; future waves can be predicted up to the physically maximal time horizon with an averaged correlation of more than $80 \%$.

\footnotetext{
${ }^{1}$ Published in this form except for references as: A.P. Wijaya et.al. Reconstruction and future prediction of the sea surface from radar observations. Ocean Eng. 106, 261-270. 2015.
} 


\section{$2.1 \quad$ Introduction}

Attempts to use remote sensing of the sea surface for prediction of the actual and future surface elevation in the vicinity of floating ships or offshore structures are motivated by various offshore and maritime engineering applications. Positioning of vessels would benefit from knowledge of the near future incoming low and high waves. Helicopter landing and loading/off-loading operations with at least one floating structure involved are examples of operations of which the critical phase (touch down or lift off) is conducted preferably during a time window with low waves. These workable time windows may occur as well in relatively high seas making their prediction very valuable to increase operational time. Knowing the approach of a freak wave, which seems to occur much more frequently than previously thought, can help to control ships in a safer way Clauss et al. 2012. An attractive option for the remote wave sensor is the nautical X-band radar. Much attention has been given since several decades to its application as a wave sensor. The vast majority of the efforts so far has been based on spectral 3D FFT methods dedicated to retrieve statistical wave parameters such as mean wave period, wave direction, non-phaseresolved directional wave spectra and properties that could be derived from the surface elevation like water depth and surface current speed and direction. Young et al. 1985 used spectral analysis to detect currents, and Ziemer and Rosenthal 1987 proposed the use of a modulation transfer function to derive surface elevation from radar images of the sea surface. Borge et al. 1999 used the signal-to-noise (SNR) ratio in radar images to propose an approximate relation for the significant wave height with two parameters that have to be calibrated. The question how to reveal the exact relation between radar images and wave elevation/significant wave height has been subject to many more publications, see e.g. Buckley and Aler 1998b and Gangeskar 2014. We will not address this topic here, but refer to a forthcoming publication of Wijaya and van Groesen 2016] that derives the significant wave height from the shadowed images without any calibration. In this paper it is assumed that the significant wave height is known, either from existing analysis techniques of radar images or by means of a reference observation such as a wave buoy or recorded ship motions.

Some of the rare attempts to retrieve the actual deterministic, i.e. phase resolved, wave surface elevation from radar-like images are reported by Blondel and Naaijen 2012 and Naaijen and Blondel 2012, but the quality was shown to be not optimal. A very different method has been explored by Aragh and Nwogu 2008; they use a 4D Var assimilation method, assimilating (raw) radar data in an evolving simulation. Nevertheless, it seems that in the literature no statistically significant evidence has been reported for successful deterministic wave sensing (reconstruction), or any method to propagate the waves to a blind area or to provide predictions.

To overcome the 'blind' zone around the radar where no elevation information is available, a propagation model is needed to evolve phase resolved reconstructed waves in the visible area into the blind zone and to make future predictions of the waves there, e.g. at the position of the ship carrying the radar antenna. The main aim of this paper is to present a scenario that integrates the inversion of the observed images with the propagation and prediction. This integration is achieved by a robust 
dynamic averaging-evolution procedure which will be shown to provide a prediction accuracy that is significantly higher than the accuracy of the observation of a single image itself.

In the following we will restrict to the case that the radar position is fixed; images from a radar on a ship moving towards the waves will require some obvious adaptations, and will reduce the prediction horizon. The complete evolution scenario takes into account the specific geometry determined by the radar scanning characteristics. For the common nautical X-band radars one can distinguish the ring-shaped area where information from radar scans is available, and the near radar area where this information is missing. Through the outer boundary of the ring, some $2000 \mathrm{~m}$ away from the radar, waves enter and leave the area; part of the incoming waves evolve towards the near-radar area or interact with waves that determine the elevation there. Hence, updates to catch the incoming waves have to be used repeatedly. The inner boundary of the ring determines the disk, the near-antenna area with a radius of some $500 \mathrm{~m}$; there no useful radar information is available because the backscatter is too high and/or suffers from interaction effects with the ship's hull. A propagation model has to evolve the information from the ring area inwards to the radar position. This description defines the main ingredients of a process that has to be developed into a practical scenario that is sufficiently efficient and accurate, noting that the quality of the simulated elevation in the near-radar area depends on the quality of the simulation in the radar ring. Since radar images give only partial and distorted information about the actual sea surface, mainly because of the shadowing effect, a phase resolved reconstruction of the sea - the inversion problem - is important. As we will show, the use of a sequence of images in a spatially dynamic scenario will predict the present and future sea surface in a reasonable degree of accuracy.

We start to propose two simple reconstruction methods for single images, but fail to reduce the effects of shadowing noticeably; consecutive simulations with the raw and the the reconstructed images will provide an indication of the robustness of the complete scenario. Indeed, the quality of the reconstruction will be substantially enhanced by the dynamic averaging and evolution procedure, almost independent of the choice of these initial images. The procedure consists of the averaging of a few successive (reconstructed) images, together with the result of the dynamic simulation, to produce updates that are assimilated in the dynamic simulation. We will use the full ring shaped observation domain surrounding the target location; this makes it possible to reconstruct and predict uni-modal wind waves as well as multi-modal seas with wind waves and swell(s) coming from possibly substantially different directions. Specific attention will be paid to the question how to treat the evolution of multi-modal seas in the proposed scenario.

In this paper we use synthetic data and make some simplifications for ease of presentation, but the scenario to be described can also be applied for more realistic cases. The use of synthetic data makes it possible to quantify the quality of the results which will be difficult to achieve in field situations for which reliable data of the surface elevation both in the ring-shaped observation area and the near-radar area simultaneously are very difficult to obtain. The wind and wind-swell seas that we synthesize are chosen to be linear to simplify the evolution, but linearity is not essential. From the synthetic seas, we construct synthetic radar images by only 
taking the geometric effect of shadowing into account as an illustration that the scenario can resolve imperfections of that kind.

The paper is arranged according to the successive steps in the proposed scenario. Section 2.2 will describe the design of (multi-modal) synthetic seas and of synthetic radar images by applying the shadowing effects. In Section 2.3 the complete dynamic averaging-evolution scenario (DAES) will be described to determine from the shadowed images the wave elevation inside the observable area and inside the blind area near the radar. Section 2.4 describes the results for two case studies, one case of wind waves, and the other one for wind-swell seas; apart from reconstruction results, the quality of predictions are investigated up to the maximal prediction time. In Section 2.5 the results of the study case are discussed and conclusive remarks will be given in Section 2.6 .

\subsection{Synthetic Data}

After a motivation to restrict the investigations to shadowed seas in the first subsection, we describe the construction of the synthetic surface elevation maps. These will be used in Section 2.2.3 to generate the synthetic geometric images that take into account the shadowing effect, and later to quantify the quality of the reconstructed and evolved surface elevations.

\subsubsection{Simplifications}

When the sea will be scanned by the radar, parts of it will be hidden for the electromagnetic radar waves since they are partly blocked by waves closer to the radar, the geometric shadowing. It should be remarked that investigations of radar data by Plant and Farquharson 2012a do not support the hypothesis that geometric shadowing plays a significant role at low-grazing-angle; indications are found that shadowing rather occurs as so-called partial shadowing. Besides shadowing, tilt (slope of the sea surface relative to the look-direction of the radar) is considered to be an important modulation mechanism for wave observations by radar, see Borge et al. 2004] and Dankert and Rosenthal 2004]. In all these references the so-called hydrodynamic modulation as described by e.g. Alpers et al. 1981 has been ignored. Possible other effects perturbing the observation that are introduced by specific hardware related properties of a radar system should in general be invertible when the exact properties are known, which is why we do not consider that aspect here.

In this paper we will consider as example of imperfections of the observed sea the effect of geometric shadowing. For this relevant effect it will be shown how well the proposed averaging-evolution scenario can cope with imperfections with a length scale of the order of one wavelength, virtually independent of the precise cause of the imperfections. Since this geometrical approach is mainly valid as a first order approach of the backscattering mechanism for grazing incidence conditions at far range for marine radar Borge et al. 2004, electromagnetic diffraction Plant and Farquharson, 2012b is not taken into account in this paper. It must be noted that perturbations over larger areas as caused by severe wind bursts may not be recovered 
accurately by the present methods.

\subsubsection{Synthetic surface elevations}

To synthesize a sea, we use a linear superposition of $N$ regular wave components each having a distinct frequency and propagation direction. The wave spectrum $S_{\eta}(\omega)$ is defined on an equally spaced discrete set of frequencies $\omega_{n}$ covering the significant energy contributions. In order to assure that the sea is ergodic Jefferys, 1987, it is required that only a single direction corresponds to each frequency. A propagation direction is assigned to each wave component by randomly drawing from the directional spreading function which is used as a probability density function, as proposed by Goda [2010]. The directional spreading function with exponent $s$ around the main direction $\theta_{\text {main }}$ is given by

$$
D(\theta)= \begin{cases}\beta \cos ^{2 s}\left(\theta-\theta_{\text {main }}\right), & \text { for }\left|\theta-\theta_{\text {main }}\right|<\pi / 2 \\ 0, & \text { else }\end{cases}
$$

with normalization $\beta$ such that $\int D(\theta) d \theta=1$.

With $k_{n}$ the length of the wave vectors corresponding to the frequencies $\omega_{n}$, and with $\phi_{n}$ phases that are randomly chosen with uniform distribution in $[-\pi, \pi]$, the sea is then given by

$$
\eta(\mathbf{x}, t)=\sum_{n} \sqrt{2 S_{\eta}\left(\omega_{n}\right) d \omega} \cos \left(k_{n}\left(x \cos \left(\theta_{n}\right)+y \sin \left(\theta_{n}\right)\right)-\omega_{n} t+\phi_{n}\right)
$$

Snapshots of the surface elevation at multiples of the radar rotation time $d t$ are given by $\eta(\mathbf{x}, n \cdot d t)$.

\subsubsection{Geometric Images}

With 'Geometric Images' we refer to the synthesized radar observation of the surface elevation for which, as stated above, we will only take the geometric shadowing into account. Shadowing along rays has been described by Borge et al. 2004 and is briefly summarized as follows.

After interpolating the image on a polar grid, with the radar at the origin $\mathbf{x}=$ $(0,0)$, we take a ray in a specific direction, starting at the radar position towards the outer boundary, using $r$ to indicate the distance from the radar. We write $s(r)$ for the elevation along the ray, and $h_{R}$ for the height of the radar. The straight line to the radar from a point $(r, s(r))$ at the sea surface at position $r$ is given for $\rho<r$ by $z=\ell(\rho, r)=s(r)+b \cdot(r-\rho)$ with $b=\left(h_{R}-s(r)\right) / r$. The point $(r, s(r))$ at the sea surface is visible if $\ell(\rho, r)>s(\rho)$ for all $\rho<r$, i.e. if $\min _{\rho}(\ell(\rho, r)-s(\rho))>0$. At the boundary of such intervals the value is zero, and so the visible and invisible intervals are characterized by $\operatorname{sign}\left[\min _{\rho}(\ell(\rho, r)-s(\rho))\right]=0$ and $=-1$ respectively. This leads to the definition of the characteristic visibility function as

$$
\chi(r)=1+\operatorname{sign}\left[\min _{\rho}\{\Theta(r-\rho) \Theta(\rho)(\ell(\rho, r)-s(\rho))\}\right]
$$


where $\Theta$ is the Heaviside function, equal to one for positive arguments and zero for negative arguments. The visibility function equals 0 and 1 in invisible and visible intervals respectively. The shadowed wave ray, as seen by the radar, is then given by

$$
s_{\text {shad }}(r)=s(r) \cdot \chi(r)
$$

which defines the spatial shadow operator along the chosen ray. Repeating this process on rays through the radar for each direction, leads to the shadowed sea, $S_{\text {shad }}(\mathbf{x})$. The geometric image is obtained by removing information in a circular area around the radar position with a radius of $r_{0}$. Then the geometric image is described by

$$
I(\mathbf{x})=S_{\text {shad }}(\mathbf{x}) \cdot \Theta\left(|\mathbf{x}|-r_{0}\right)
$$

\subsection{Dynamic averaging-evolution scenario}

This section presents the dynamic averaging-evolution scenario (DAES) that will provide a reconstruction and prediction of the surface elevation at the radar position using the geometrically shadowed waves in the ring-shaped observation area of the sea. The main ideas can be described as follows.

The exact (non-shadowed) sea is supposed to evolve according to a linear (dispersive) evolution operator. Except from entrance effects of waves through the boundary, one snapshot of the sea would be enough to determine exactly the whole future evolution. The effects of shadowing give a space and time dependent perturbation for all images: the amount of shadowing (visibility) depends on the distance from the radar, and the position in time of the waves determines the actual area of shadowing, shifting and changing somewhat with the progression of the wave. Hence, one snapshot of the observed (shadowed) sea will produce a different evolution result than that of the exact sea because the zero-level of the shadowed area will be evolved. In order to control, and actually reduce, the error, we use updates to be assimilated in the dispersive evolution. After three radar rotation times $3 d t$ we update the ongoing simulation by assimilation with the averaged 3 preceding images, where the averaging itself already reduces the effect of shadowing somewhat. Since we do this globally, so also in areas closer to the radar where the shadowing is less severe, the result with the dynamic averaging-evolution scenario shows that this is sufficiently successful to give an acceptable correlation in the radar area.

The first subsection deals with two simple methods that aim to improve the quality of each individual geometric image by attempting to fill in the gaps caused by the shadowing. Then the evolution of a single image is discussed in some detail, after which the dynamic averaging of several images is described to construct updates that will be used in subsection four as assimilation data in an evolution of the full sea.

\subsubsection{Spatial reconstruction of geometric images}

In the following, two methods will be presented for a first attempt to reconstruct the geometric images in regions where the observation is shadowed. In the first method 
the geometric image is shifted vertically such that the spatial average (over the observation area) vanishes. With a scaling factor $\alpha$ to obtain the correct significant wave height, this will produce the reconstructions $R_{n}^{1}$ as

$$
R_{n}^{1}(\mathbf{x})=\alpha\left(I_{n}(\mathbf{x})-\operatorname{mean}\left(I_{n}\right)\right)
$$

As mentioned in the Introduction 2.1, it is assumed that the true variance of the waves (or significant wave height) is known from either additional analysis and/or a reference measurement so that $\alpha$ is determined.

The second proposed method is described as

$$
R_{n}^{2}(\mathbf{x})=\alpha\left(I_{n}(\mathbf{x})-E\left(I_{n},-T / 2\right)\right)
$$

Here $E\left(I_{n},-T / 2\right)$ evolves the sea backwards in time over half of the peak period, for which in multi-modal seas we will take the peak period of the wind waves. The evolution indicated here with the operator $E$ will be explained in detail in the next subsection. Note that for harmonic long crested waves with period $T$ of which negative elevations have been put to zero elevation (to roughly resemble the effect of shadowing) leads to the correct harmonic wave by the reconstruction $R^{2}$.

\subsubsection{Evolution of a single image}

Let the reconstructed geometric image, denoted by $R$, obtained by either reconstruction method described in the previous subsection, be given by its $2 \mathrm{D}$ Fourier description as:

$$
R(\mathbf{x})=\sum_{\mathbf{k}} a(\mathbf{k}) e^{i \mathbf{k} \cdot \mathbf{x}}
$$

Here $\mathbf{k}$ is the $2 \mathrm{D}$ wave vector, and the coefficients $a$ can be obtained by applying a 2D FFT on $R$.

The image itself is not enough to define the evolution uniquely since the information in which direction the components progress with increasing time is missing. For given direction vector e, define the forward evolution as

$$
E_{\mathbf{e}}(R, t)=\sum_{\mathbf{k}} a(\mathbf{k}) \exp i[\mathbf{k} \cdot \mathbf{x}-\operatorname{sign}(\mathbf{k} \cdot \mathbf{e}) \Omega(k) t]
$$

where $k=|\mathbf{k}|$ and $\Omega(k)=\sqrt{g k \tanh (k D)}$ is the exact dispersion above depth $D$. Waves propagating in a direction $\tilde{\mathbf{e}}$ that makes a positive angle with $\mathbf{e}$, so $\tilde{\mathbf{e}} \cdot \mathbf{e}>0$, will then propagate in the correct direction for increasing time, which justifies to call the evolution forward propagating with respect to e. Changing the minus-sign into a plus-sign in the phase factor, the backward propagating evolution in the direction $-\mathbf{e}$ is obtained.

For uni-modal sea states, such as wind waves or swell, there will be a main propagation direction $\mathbf{e}_{\text {prop }}$, which is the direction of propagation of the most energetic waves. Other waves in such wave fields will usually propagate in nearby directions, under an angle less than $\pi / 2$ different from the main direction. In such cases we can take $\mathbf{e}_{\text {prop }}$ as the direction to define the evolution. Actually, any direction from 
the dual cone of wave vectors can be chosen, i.e. any vector that has positive inner product with all wave directions.

In multi-modal sea states, in most practical cases a combination of wind waves and swell, the situation is different since the waves may have a wider spreading than the $\pi / 2$ difference from the main direction that was assumed for the unimodal sea states. When the wave directions are spread out over more than a half space, one evolution direction so that all waves are propagated correctly cannot be found anymore. If only low-energy waves are outside a half space, one may still use a forward propagating evolution operator. Then an optimal choice is the main evolution direction for which the maximum portion of the total wave energy is evolved correctly. A way to identify this optimal direction is discussed now.

Practically, we use a second (or more) 'control' image, and look for which vector e the evolution of the first image corresponds with the control image as good as possible in least-square norm; this then determines the main evolution direction (MED). Explicitly, given two successive images of the wave field, say $R_{1}$ and $R_{2}$ a small time (the radar rotation time) $d t$ apart, we compare $R_{2}$ with the forward evolution of $R_{1}$ over time $d t$ in the direction $\mathbf{e}$, to be denoted by $E_{\mathbf{e}}\left(R_{1}\right)$, and minimize the difference over all directions e, defining the MED as the optimal value

$$
\mathbf{e}_{M E D} \in \min _{\mathbf{e}}\left|E_{\mathbf{e}}\left(R_{1}\right)-R_{2}\right|
$$

Instead of minimizing a norm of the difference, one can also take the maximum of the correlation. For fields with limited directional spreading there will be a broad interval of optimal directions, in which case the average of the optimal directions can be chosen. For cases of multi-modal sea states where the main propagation direction of the different modes deviates very much there is likely to be one distinct optimal MED. It is possible that with this method using the MED, a significant amount of wave energy is evolved in the wrong direction, depending on how much the main directions of the different modes differ.

In the following we will use a simplified notation when evolving over one time step $d t$, namely

$$
\mathcal{E}(R)=E_{\mathbf{e}_{M E D}}(R, d t)
$$

Evolving over several time steps, say $m . d t$, is then written as a power (succession of evolution) $\mathcal{E}^{m}$.

\subsubsection{Updates from dynamic averaging}

The reconstruction process described in Section 2.3.1 gives approximate sea states $R_{n}$. The study cases will show that these reconstructions are still rather poor when compared to the exact synthetic surface elevation maps; the correlation with the exact surface is only slightly better than that for the shadowed geometric images. In order to reduce the effect of this reconstruction error and thereby to improve the accuracy of the elevation prediction near the radar, we propose an averaging procedure in physical space. This procedure will involve three successive reconstructed images and the simulated wave field at the instant of the last image. 
To set notation, the simulated sea (the simulation process will be detailed below) at time $t$ will be denoted as $\zeta(\mathbf{x}, t)$; at discrete times $m . d t$ we write $\zeta_{m}(\mathbf{x})=$ $\zeta(\mathbf{x}, m . d t)$. The simulation is initialized by taking for the first three time steps the three successive reconstructed images

$$
\zeta_{m}(\mathbf{x})=R_{m}(\mathbf{x}) \text { for } m=1,2,3
$$

For the continuation, updates will be used to assimilate the evolution. We describe the update process at a certain time $t_{0}$, which is a multiple of $3 d t$. Available at that time is the simulated wave field at $t_{0}$, to be denoted by $\zeta_{0}(\mathbf{x})=\zeta\left(\mathbf{x}, t_{0}\right)$, the reconstructed image at time $t_{0}$, and 2 previous images at times $t_{-1}=t_{0}-d t, t_{-2}=$ $t_{0}-2 d t$; these reconstructed images will be denoted by $R_{0,-1,-2}$ respectively. Since the images $R_{k}$ have substantial inaccuracies despite the reconstruction, it can be expected that an averaging procedure improves the quality. This averaging has to be done in a dynamic way to compensate for the fact that the images are available at different instants in time. Therefore the images $R_{-1}$ and $R_{-2}$ have to be evolved over one, respectively two, time steps $d t$. This produces $\mathcal{E}\left(R_{-1}\right)$ and $\mathcal{E}^{2}\left(R_{-2}\right)$, each representing, just as $R_{0}$, an approximation of the sea state at time $t_{0}$. But the information will be different, partly complementary, because the information at different time steps shows somewhat different parts of the wave because of the shadowing effect. Therefore an arithmetic mean will contain more information, and may also reduce incidental errors and noise. The ongoing simulation $\zeta_{0}$ also gives an approximation of the sea at $t_{0}$, and, most important, will also contain elevation information in the near-radar area where the $R_{k}$ are vanishing. Choosing some weight factors, we therefore take as update at time $t_{0}$ the following combination

$$
U_{0}(\mathbf{x})=\left(\frac{1}{6}\left(R_{0}+\mathcal{E}\left(R_{-1}\right)+\mathcal{E}^{2}\left(R_{-2}\right)\right)+\frac{1}{2} \zeta_{0}\right)\left(1-\chi_{\text {rad }}\right)+\zeta_{0} \chi_{\text {rad }}
$$

Here $\chi_{\text {rad }}(\mathbf{x})$ is the characteristic function (or a smoothed version) of the near-radar area: $\chi_{\text {rad }}=1$ in the near radar zone where no waves can be observed and $\chi_{\text {rad }}=0$ in the remaining area. The number of reconstructed images to be taken in the update can be more or less than 3 , and each could be given a different weight. Our experience with various test cases led to the weight factors as taken above.

\subsubsection{Evolution and prediction}

The updates defined above will be used as assimilation data to continue the simulation. In detail, after the construction of an update, say $U_{3 m}$, the simulation continues with this sea state as initial elevation field for three consecutive time steps:

$$
\zeta_{3 m+j}=\mathcal{E}^{j}\left(U_{3 m}\right) \text { for } j=1,2,3 .
$$

This defines the full evolution in time steps $d t$, which is repeatedly fed with new information from the reconstructed images through the updates. This scenario can run in real time in pace with incoming real radar images.

A prediction can be defined, starting at any time $t_{0}=m$. $d t$ for a certain time interval ahead, without using any information of geometric images later than $t_{0}$. 
The prediction, say for a future time of $\tau \in[0, \Pi]$, where $\Pi$ is the prediction horizon, is then defined as

$$
P\left(t_{0}, \tau\right)=E\left(\zeta\left(t_{0}\right), \tau\right) \text { for } \tau \in[0, \Pi]
$$

An upper bound for the prediction horizon depends on the speed of the waves and the distance of the outer boundary to the radar. As shown by $\mathrm{Wu} 2004$ and Naaijen et al. 2014 the prediction horizon is mainly governed by the group velocity of the waves and the size of the observation domain. In case of a nautical radar, the spatial observation domain will be the ring-shaped area, previously indicated by $\chi_{\text {rad }}=0$. The group velocity will be different and in different directions for short-crested, in particular multi-modal, seas and depend on wave characteristics (roughly the peak period) and the depth. These factors will influence the prediction horizon in which we can expect a reliable prediction. Besides that the prediction horizon $\Pi$ clearly also depends on the accuracy that is desired for the prediction.

\subsection{Case studies}

In this section we present the results for two study cases: one for wind waves and one with combined wind waves and swell. Comparisons are presented between the predicted wave elevation, obtained by processing the synthesized images with the proposed DAES method and the exact wave elevation as it was synthesized. We start to specify the sea data and other physically and numerically relevant parameters of the simulations, followed by the simulation results.

\subsubsection{Parameters of the study cases}

\section{Geometry and spatial grid parameters}

The seas that we consider evolve above a depth $h=50 \mathrm{~m}$. The height of the radar is an important quantity because the severity of the shadowing effect is governed by the ratio of radar height and wave height. We will report on a value of the radar height $h_{R}$ of $15 \mathrm{~m}$ above the still water level. The radar is assumed to be at a fixed position above the still water level, with a constant radar rotation speed $d t=2 \mathrm{~s}$. The sea is constructed in an area $[-2050,2050]^{2}$ with a number of nodes in the $x$ and the $y$-direction equal to $N_{x}=N_{y}=512$, so spatial step size $d x=d y=7.9$ $\mathrm{m}$. Modeling the outer boundary of the radar observation area, the elevation of each snapshot of the sea is made to vanish for distances from the radar larger than $r_{\max }=1800 \mathrm{~m}$. The shadowing procedure is applied after transforming each sea state to polar coordinates $(r, \phi)$ on a grid with $d r=7.5 \mathrm{~m}$ and $d \phi=0.3^{\circ}$. The geometric image is then obtained by transforming back to Cartesian coordinates and make the elevation vanish in the circular near radar area of radius $r_{\min }=500$ $\mathrm{m}$. 


\section{Sea states}

We provide the properties of the wind waves and the swell separately; since we consider linear waves, the characteristics of the multi-modal sea state, which is a combination of the wind waves and swell, can be derived in a straightforward way. The properties of the waves, with related wave characteristics above depth $h=50$ $\mathrm{m}$, are summarized in Table 2.1 .

Table 2.1: Characteristic of sea and swell waves

\begin{tabular}{ccccccccccc}
\hline Sea & $H_{s}$ & $T_{p}$ & $\gamma$ & $\theta_{\text {main }}$ & $s$ & $\omega_{p}$ & $k_{p}$ & $\lambda_{p}$ & $C_{p}$ & $V_{g}$ \\
\hline Wind & 3 & 9 & 3 & $-\pi / 2$ & 10 & 0.7 & 0.05 & 125 & 13.9 & 7.4 \\
\hline Swell & 1 & 16 & 9 & $3 \pi / 4$ & 50 & 0.4 & 0.02 & 308 & 19.2 & 14.8 \\
\hline
\end{tabular}

The wind waves have main propagation direction from North to South, $\theta^{W}=$ $-\pi / 2$; the wave spreading is given by the spreading function 2.1 with exponent $s=10$. The frequency spectrum of the wind waves is a Jonswap spectrum with $\gamma=3$, peak period $T_{p}=9 \mathrm{~s}$, and significant wave height $H_{s}^{W}=3 \mathrm{~m}$. Note that the significant wave height is an important factor that affects the amount of shadowing; the ratio of radar height and significant wave height is as low as 5 in this study case, leading to substantial shadowing.

The multi-modal sea consists of the above wind waves to which is added the swell waves. The swell consists of waves from the South-Eastern direction, $\theta^{S}=3 \pi / 4$, peak enhancement factor $\gamma=9$, wave spreading with $s=50$, peak period $T_{p}=16 \mathrm{~s}$, and significant wave height $H_{s}^{S}=1 \mathrm{~m}$. The significant wave height of this combined sea state will be $H_{s}^{W S}=\sqrt{10} \approx 3.15 \mathrm{~m}$, so that the ratio of radar height and significant wave height is slightly less than 5 .

The study cases of wind waves without swell and combined wind waves-swell will be denoted by W15 and WS15 respectively. The number of discrete components $N$ used to synthesize the waves as in Eq. (2), has been taken $N=1500$ for the wind waves and $N=700$ for the swell in study case WS15.

\section{Main evolution direction}

As described in Section 2.3.2, the main evolution direction MED will be determined as the direction for which the error of the difference between a one-step evolved image and the successive image is as small as possible. For the study cases Fig. 2.1 shows the averaged relative error obtained by comparing 10 pairs of successive reconstruction images for case W15 and WS15. Here, the angle is measured from the positive $x$-axis in counter clockwise direction. For study case W15 the relative error is rather constant in the interval $\left[-150^{\circ},-30^{\circ}\right]$, with $-90^{\circ}$ in the middle of the interval. Hence this is chosen as MED, which coincides with the design value of the main wind direction of the synthesized wave field. For case WS15 the situation is very different. There is now only a small interval of angles identifying evolution directions for which most energy is propagated correctly. Hence, for case WS15 the angle of minimal error is chosen as MED, i.e. $-148^{\circ}$. For the study cases using the shadowed images to determine MED we observed a few degrees difference with the 
MED's found when using the synthetic non-shadowed seas; in the following we take the values obtained from the shadowed seas.

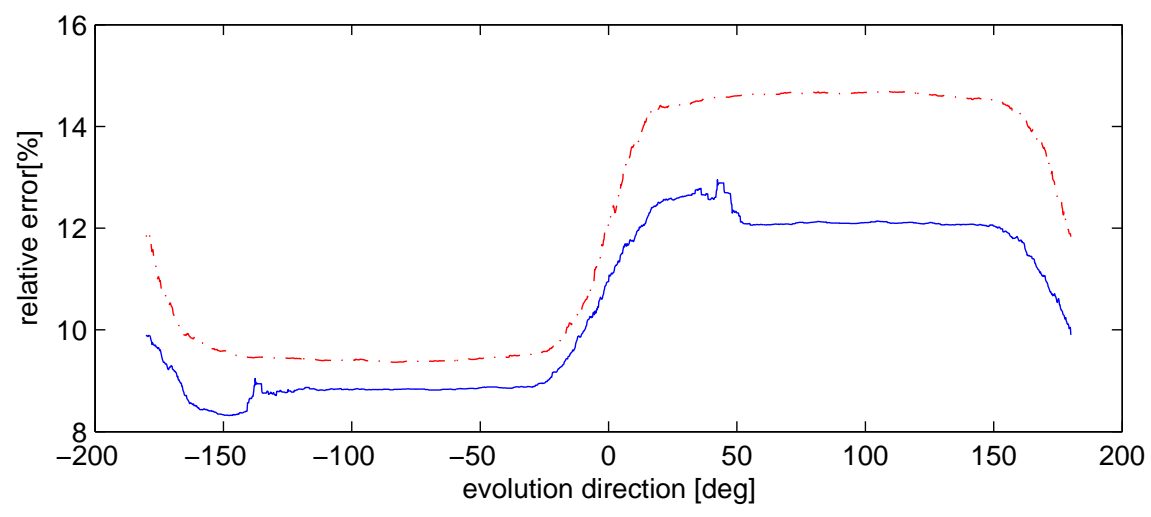

Figure 2.1: The relative error in the procedure to determine the main evolution direction MED averaged over 10 realizations, for case W15 (dash-dotted red) and WS15 (solid blue).

\subsubsection{Simulation results}

In this paragraph results of the simulations will be described. After some graphical presentations, more quantitative information is presented for the reconstruction sea states and the future prediction.

\section{Graphical presentation}

We start with some results that illustrate the DAES method. After the first three synthesized geometric images, the dynamic averaging - evolution scenario is initiated using updates at every time that is a multiple of $3 d t$. For a certain $t=t_{0}$, shortly after starting the simulation, various images are presented in Fig. 2.2. Fig. 2.2 shows the true wave elevation as synthesized at $t=t_{0}$. Fig. $2.2 \mathrm{p}$ shows the shadowed image of the wave elevation depicted in Fig. 2.2 a with vanishing elevation in the blind area $r<500$ around the antenna. Fig. $2.2 \mathrm{k}$, shows the reconstruction $U_{0}\left(t_{0}\right)$ (also denoted by $P\left(t_{0} ; \tau=0\right)$ ). As can be seen, the wind waves propagating in the main direction from North to South in the negative $y$-direction, and more so the swell from SE to NW, have evolved already some small distance into the nearantenna zone. Fig. 2.2 d shows the reconstruction $P\left(t_{1} ; \tau=0\right)$ for a larger value $t_{1}$ at which the waves have evolved so much that they occupy the entire blind area near the antenna $r<500$. Fig. 2.3 shows the cross section in the $y$ direction at $x=0$ of the shadowed waves in Fig. 2.2 b. Different from Fig. 2.2 b, the waves are shown here for $r<r_{\min }$ as well.

As can be observed for this particular wave condition and quotient of radar altitude and significant wave height of $15 / 3$, the shadowing is rather severe: beyond $r=500$ hardly any wave troughs are visible. Despite this poor quality of the observation, the DAES procedure produces a reconstruction of the wave elevation 
a

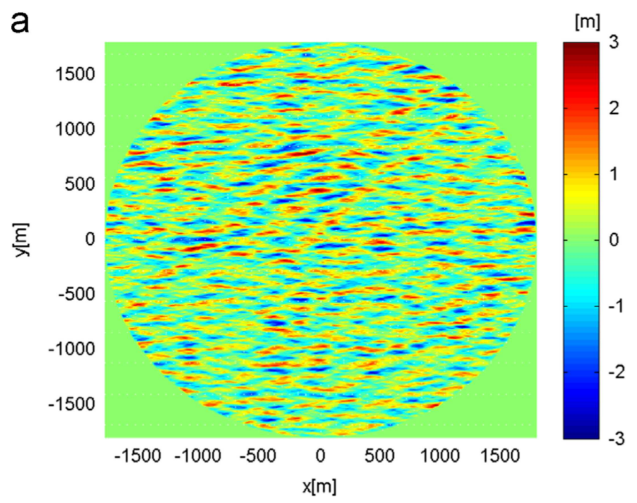

C

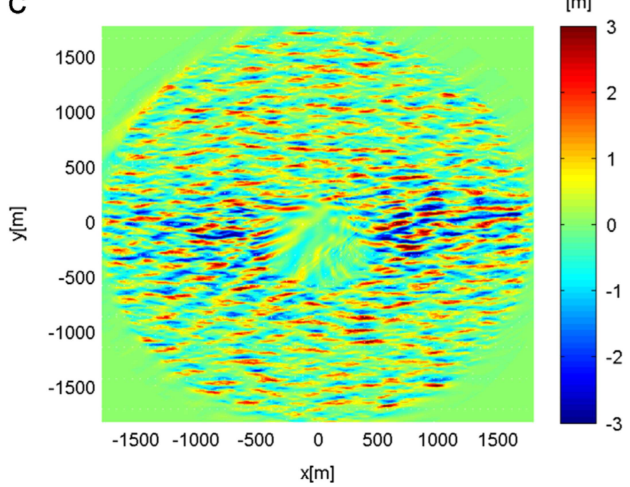

$\mathrm{b}$

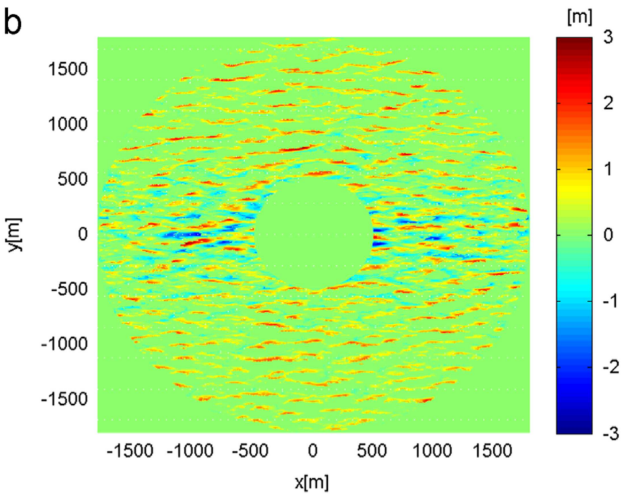

d

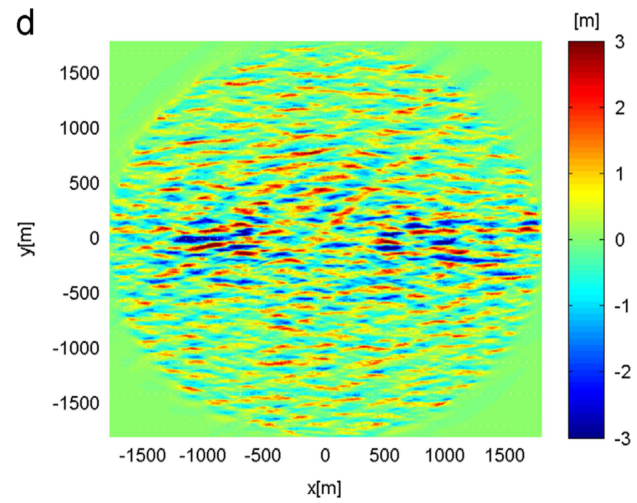

Figure 2.2: Images of the combined sea WS15 with wind waves from the North and swell from SE. Image (a) shows the real sea, and (b) the shadowed sea at the same instant. Image (c) shows the elevation shortly after the start of the simulation when the waves do not yet fully occupy the blind near radar area; at a later time, image (d) shows that the blind area has been filled with waves through the dynamic averaged evolution scenario. 


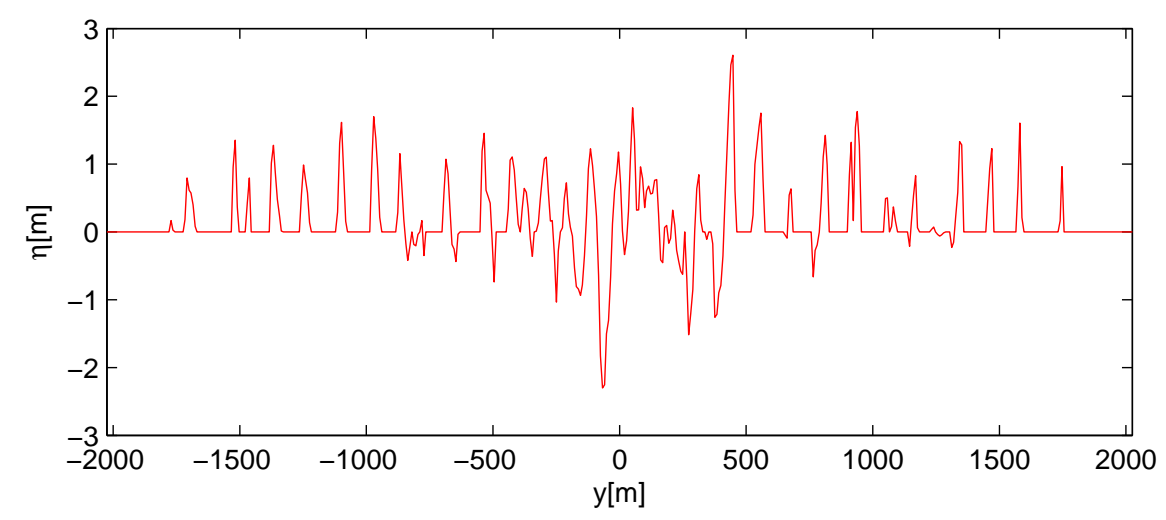

Figure 2.3: A cross section coinciding with the $y$-axis shows the shadowed waves (wind waves from right to left); observe the severe shadowing outside the blind area (-500,500).

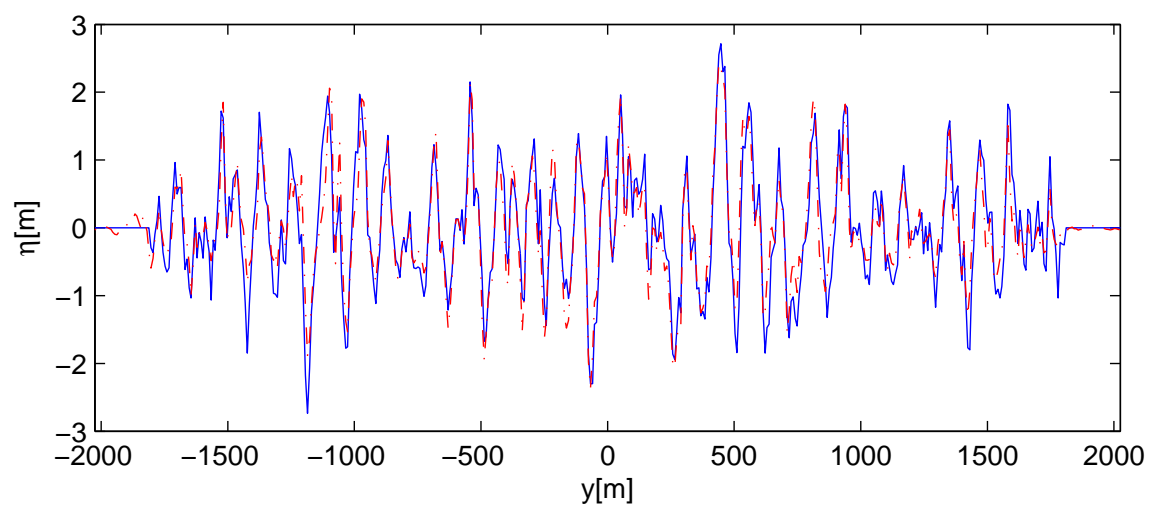

Figure 2.4: Cross section along y-axis showing the true elevation (blue,solid) and the reconstructed elevation $R^{1}$ (red, dashed).

as shown in Fig. 2.4. This figure shows plots of the synthesized elevation, referred to as "true wave", and the reconstruction $P\left(t_{1} ; \tau=0\right)$ obtained by DAES at a time $t_{1}$ such that the simulation has already run sufficiently long for the reconstructed waves to fill the entire blind zone. Observe that the reconstruction is better near the radar, near $y=0$, than for larger distances from the radar where the dynamic averaging cannot yet sufficiently improve the poor quality of the observation data near the edge of the domain.

Fig. 2.5 shows time traces of the $R^{1}$-reconstructed elevation and the true elevation at the radar position for WS15. The entrance effect at early times when the wind-waves and swell have not yet completely arrived at the radar position is clearly visible. This figure indicates that the entrance effect is visible until approximately $80 \mathrm{~s}$, which is close to the time that is needed for the most energetic wind waves to travel with group speed $7.4 \mathrm{~m} / \mathrm{s}$ from the inner ring of radius $500 \mathrm{~m}$ to the radar. 

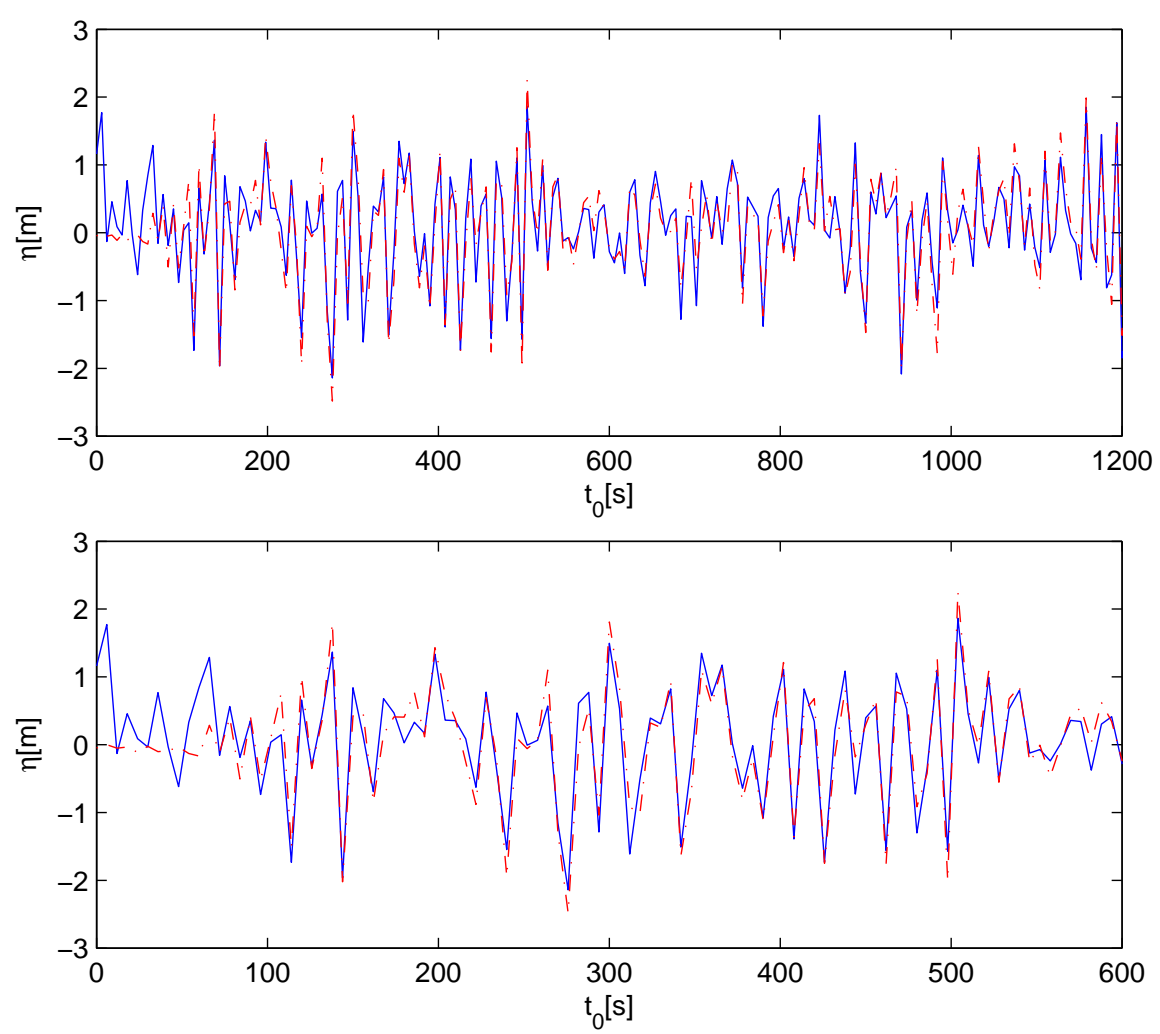

Figure 2.5: Time traces of elevation at the radar position for case WS15. In blue (solid) the true elevation, in red (dash-dotted) the reconstruction $R^{1}$ that started at time 0 . The enlarged lower plot from 0 to $300 \mathrm{~s}$ shows the entrance effect that only after some $80 \mathrm{~s}$ the faster and slower waves reached the radar position to obtain sufficient accuracy.

\section{Correlation as measure for accuracy}

The accuracy of the reconstruction and prediction is quantified by the correlation coefficient Corr, which correlates the wave elevation at one instant obtained from the simulation ('simul') with the synthetic wave elevation ('data') at the same instant according to

$$
\operatorname{Corr}(\text { data }, \text { simul })=\frac{<\text { data, simul }>}{\mid \text { data }|| \text { simul } \mid}
$$

Here $<,>$ denotes the inner product over space $\mathbf{x}$. Note that Corr defined in this way is related to the normalized point square error according to

$$
\frac{\mid \text { data }- \text { simul }\left.\right|^{2}}{\mid \text { data }|\cdot| \text { simul } \mid}=\frac{\mid \text { data } \mid}{\mid \text { simul } \mid}+\frac{\mid \text { simul } \mid}{\mid \text { data } \mid}-2 \operatorname{Corr}(\text { data }, \text { simul })
$$

In particular when 'data' and 'simul' have the same norm, it holds

$$
\frac{\mid \text { data }- \text { simul }\left.\right|^{2}}{\mid \text { data }\left.\right|^{2}}=2(1-\operatorname{Corr}(\text { data }, \text { simul }))
$$


The correlation will also be used to quantify the quality of future predictions. Using the notation $P\left(t_{0}, \tau\right)$ introduced in Eq. 2.14 for the predicted wave elevation starting with the reconstruction at time $t_{0}$ a time $\tau$ ahead, and denoting by $\eta\left(t_{0}+\tau\right)$ the synthetic wave elevation from Eq. 2.2, their spatial correlation will be denoted by

$$
c\left(t_{0}, \tau\right)=\operatorname{Corr}\left(P\left(t_{0}, \tau\right), \eta\left(t_{0}+\tau\right)\right) \text {. }
$$

Then in order to obtain a statistically more reliable average correlation coefficient corr, the average is taken over an interval of $t_{0}$ values:

$$
\operatorname{corr}(\tau)=\frac{1}{J} \sum_{j=1}^{J} c\left(t_{0 j}, \tau\right)
$$

To avoid entrance effects, the computation of $\operatorname{corr}(\tau)$ is restricted to times $t_{0}$ such that all waves have evolved to fill completely the blind zone. For the presented simulations, this distance (of $1000 \mathrm{~m}$ ) is covered by the wind waves with group speed at peak frequency in approximately $136 \mathrm{~s}$, i.e. $68 d t$; for the swell waves with double group speed, this time is $68 \mathrm{~s}$. The number of simulation steps $J$ used for calculation of $\operatorname{corr}(\tau)$ has been at least 200 for all presented results.

\section{Accuracy of reconstruction}

The correlation has been computed for both sea states W15 and WS15, for various sizes of the spatial domain: corr is determined for $r<50, r<500$ and $r>500$. Results are presented in Tables 2.2 and 2.3 for the 'reconstruction', i.e. $\tau=0$; prediction results for which $\tau>0$ will be presented in the next paragraph.

The first column in Tables 2.2 and 2.3 indicates the type of input data used in the DAES procedure. 'Sea' refers to the perfect (not shadowed) synthetic waves as input images, but with vanishing elevation in the near radar area $r<500$. In this column $R^{0}$ refers to simulations with shadowed waves without applying any reconstruction of the individual images, while $R^{1}$ and $R^{2}$ refer to the two reconstruction methods as defined in Section 2.3.1. The columns with 'Raw' and 'Rec' show the correlation of the geometric images and the individually reconstructed images with the true wave elevation respectively; the area over which the correlation is taken is the outer ring area $500<r<1800$.

Table 2.2: Correlation for W15 averaged over time for various reconstruction methods.

\begin{tabular}{cccccc}
\hline & Raw & Rec & $r<50$ & $r<500$ & $r>500$ \\
\hline Sea & 1.00 & 1.00 & 0.99 & 0.99 & 1.00 \\
$R^{0}$ & 0.71 & 0.71 & 0.82 & 0.87 & 0.83 \\
$R^{1}$ & 0.71 & 0.75 & 0.95 & 0.95 & 0.89 \\
$R^{2}$ & 0.71 & 0.75 & 0.89 & 0.91 & 0.84 \\
\hline
\end{tabular}

As illustration, for a typical case, the correlation between the true sea and the $R^{1}$-reconstruction in the radar area $(r<50 \mathrm{~m}$ and $r<200 \mathrm{~m})$ is given in Fig. 2.6 as function of increasing time during the DAES process. The entrance effect is 
Table 2.3: Same as Table 2.2 now for bi-modal sea state WS15.

\begin{tabular}{cccccc}
\hline & Raw & Rec & $r<50$ & $r<500$ & $r>500$ \\
\hline Sea & 1.00 & 1.00 & 0.99 & 0.99 & 1.00 \\
$R^{0}$ & 0.70 & 0.70 & 0.85 & 0.88 & 0.83 \\
$R^{1}$ & 0.70 & 0.74 & 0.95 & 0.95 & 0.89 \\
$R^{2}$ & 0.70 & 0.73 & 0.89 & 0.90 & 0.83 \\
\hline
\end{tabular}

clearly visible just as in Fig. 2.5 the waves need approximately $160 \mathrm{~s}$ to fill up the near-radar area of radius $200 \mathrm{~m}$.

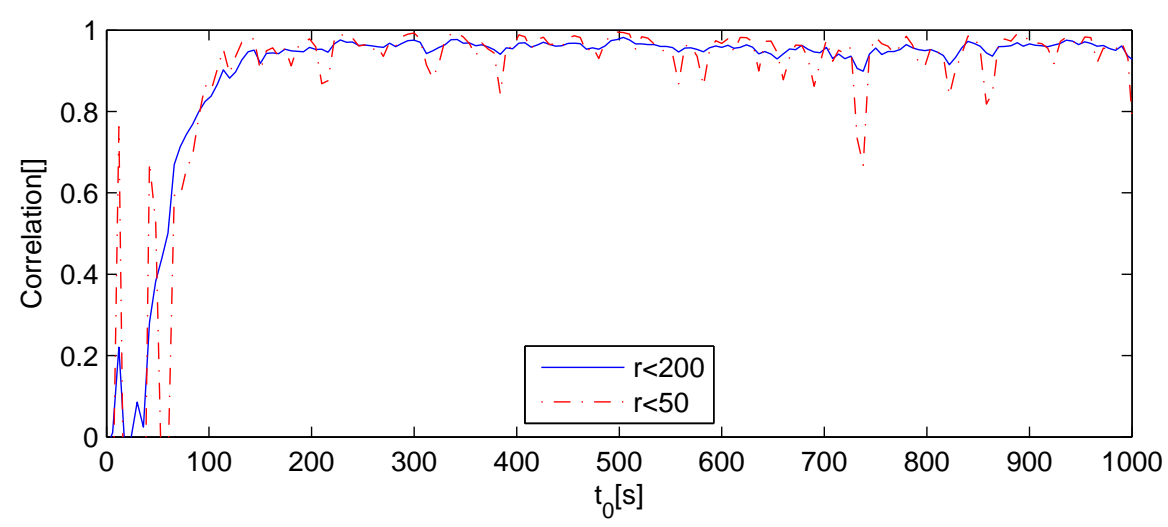

Figure 2.6: Correlation between true sea and the $R^{1}$-reconstruction for case WS15 in the radar area with radius $200 \mathrm{~m}$ (blue, solid) and radius $50 \mathrm{~m}$ (red, dash-dotted) at times after the start of the reconstruction. Observe that after some $160 \mathrm{~s}$ the reconstruction has filled these regions and becomes more accurate.

\section{Accuracy of prediction}

The eventual aim of the simulation scenario is to predict in future time the elevation in the near-radar area. At each time $t_{0}$ during the simulation, the obtained reconstruction at that time $P\left(t_{0}, \tau=0\right)$ can be taken as initial state for a prediction according to Eq. 2.14, without new updates. In Fig. 2.7 is shown a prediction at the radar position for the sea state WS15 with reconstruction method $R^{1}$. For an initial time $t_{0}>160$ larger than the filling time of the near-radar area, the predicted wave elevation and the true wave elevation at the radar position are shown as function of prediction time $\tau$.

Figs. 2.8 and 2.9 show results for prediction based on DAES applied to the true sea (perfect non-shadowed waves) and the $R^{1}$-reconstruction for case W15 and WS15 respectively. As expected, for increasing prediction time the correlation decreases. Prediction of the wind waves W15 can be done for a time horizon of 2.9 minutes with correlation above 0.9 , and for 3.6 minutes with correlation above 0.8 ; for the combined wind-swell waves WS15 these times are 2 minutes and 3.3 minutes 


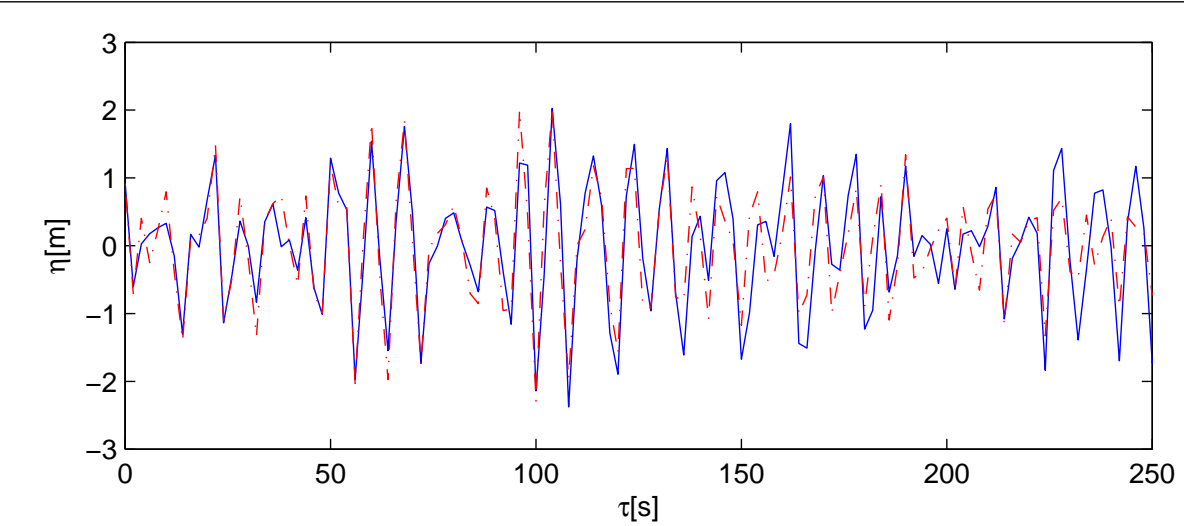

Figure 2.7: For WS15, the figure shows the prediction (red, dash-dotted) of the elevation compared to the true elevation (blue, solid)at the radar position; observe that after 120 s the prediction becomes less accurate.

respectively. Observe the steeper decrease in the graphs of WS15 after $120 \mathrm{~s}$, which is approximately the travel time of swell waves at peak group velocity; hence after that time, swell waves are not present in the prediction anymore.

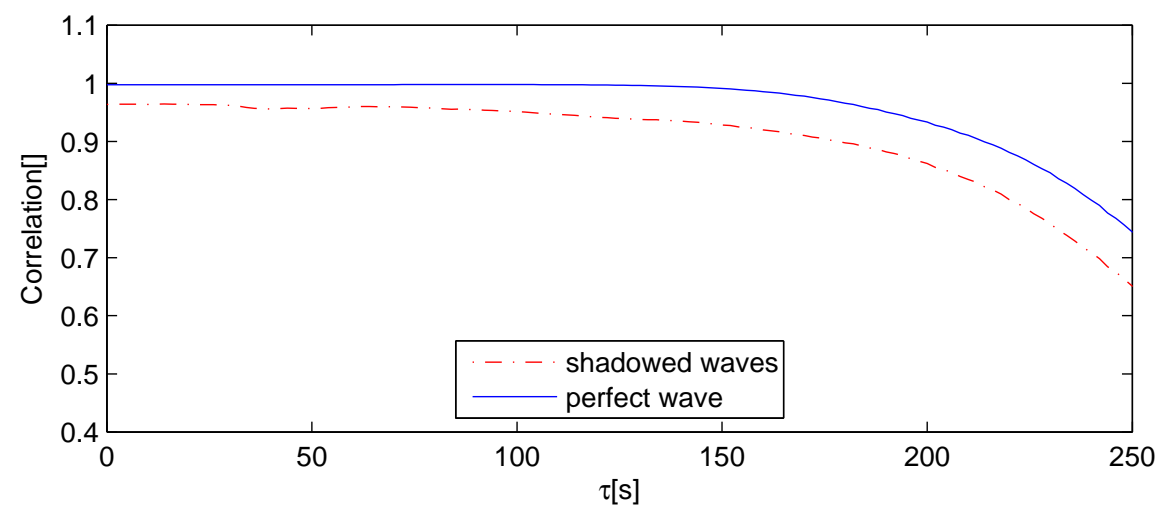

Figure 2.8: Correlation between predicted and true elevations in a radar area of radius $200 \mathrm{~m}$ using as input in the prediction method the true sea (blue, solid) and the shadowed sea of W15 (red, dash-dotted).

\subsection{Discussion of results}

\subsubsection{Reconstruction method}

The high correlations in Tables 2.2 and 2.3 for the case of a perfect 'Sea' (the nonshadowed synthetic waves) as input show that the dynamic averaging procedure 


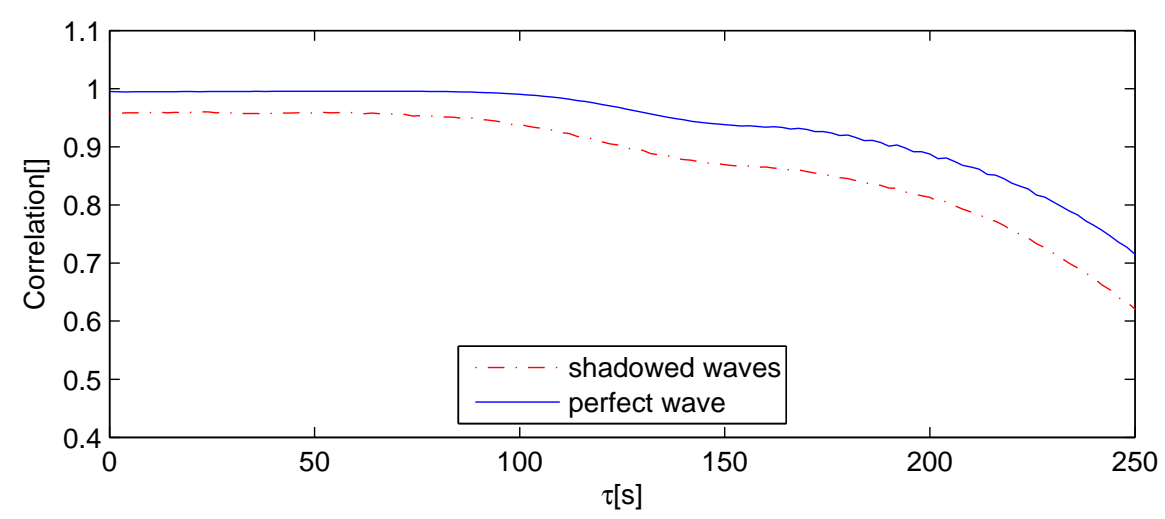

Figure 2.9: Same as Fig. 2.8 now for WS15.

and the evolution to fill the near-radar area $r<500$ proceeds almost perfectly. The tables also show that the reconstruction of each single image only slightly improves the correlation, at most $4 \%$ for $R^{1}$ and $R^{2}$. For all three individual reconstructions, the DAES improves the reconstruction substantially, with best results for the vertical shifting method $R^{1}$, for which the correlation increases from 0.75 in the outer ring to 0.95 in the near-radar area.

The comparison of the $R^{1}$-reconstructed and true elevation in Fig. 2.6 shows that variations of the correlation over the larger disc of radius $200 \mathrm{~m}$ are much smaller than over the $50 \mathrm{~m}$ disc; this may be due to a poor reconstruction of relatively small areas in the outer ring $500<r<1800$.

\subsubsection{Predictability}

The results in Figs. 2.8 and 2.9 show the capabilities and limitations of the prediction. The physically maximal prediction time can be roughly estimated as the travel time from the outer region towards the radar $(1800 \mathrm{~m})$ for the most energetic waves at peak frequency. Using the value of the group velocity of the wind waves of 7.4 $\mathrm{m} / \mathrm{s}$, this leads to a maximal prediction horizon of $240 \mathrm{~s}$ for study case W15; this seems to be a too high estimation since Fig. 2.8 shows a rather low correlation of 0.7 at that time for the best possible prediction with the true sea.

On the other hand, for the combined wind-swell sea, a similar reasoning based on the speed of swell waves is too pessimistic for the study case WS15: the correlation of prediction with the true sea is around 0.9 at that time. This can be explained by the fact that in the study case the swell waves have approximately $10 \%$ of the energy of the wind waves, which causes that the wind waves dominate the correlation, which is only slightly less than for W15 until $250 \mathrm{~s}$, despite the fact that the effects of swell are actually absent after $120 \mathrm{~s}$. The swell effect can also be observed by comparing the predicted elevation with the true elevation at the radar position as depicted in Fig. 2.7 the amplitude prediction becomes less accurate after around $120 \mathrm{~s}$ although the phase is still captured quite well for longer times. 


\subsubsection{Scaling}

The observation from Fig. 2.7 that the variance of the predicted wave elevation decreases with increasing $\tau$ is also due to the fact that for values of $\tau$ further into the future, the waves arriving at the radar location originate from further distances where the shadowing is more severe and the variance of the observation is lower; after sufficiently long time no wave information will be available at all anymore. Using one scaling factor $\alpha$ based on the variance of the entire observed image and the true variance of the waves as was proposed in Eq. 2.6. does not take into account this decreased visibility at large ranges from the radar and in fact does not even guarantee a correct variance at the radar for $\tau=0$. An alternative which is supposed to be practical and feasible for real life applications is proposed by Naaijen and Wijaya 2014]: a time history of the wave elevation at the radar position (e.g. by an auxiliary wave buoy or via recorded ship motions) and a time history of the predicted wave elevation can be recorded and used to calculate the variance of the true waves and the prediction. By taking the ratio of these variances, a scaling factor dedicated for the radar location can be obtained. Such a scaling factor can also be computed as a function of $\tau$, thus removing the aforementioned effect of decreasing variance of the prediction with increasing $\tau$.

\subsubsection{MED and bimodal sea state}

In Section 2.3.2 it was explained how the wave components obtained from a 2D FFT are propagated in the main evolution direction (MED). In case of multi-modal sea states, it depends on the difference between the propagation directions of the various modes how much of the total wave energy represented by the obtained components is propagated in the correct direction. The sea state WS15 was designed in such a way that the amount of energy represented by wave components propagating in opposite directions relative to the total wave energy is very limited which may explain the small differences in the obtained accuracy between W15 and the multi-modal case WS15. Multi-modal seas with substantial counter propagating waves require an evolution method that takes into account a splitting of waves in two opposite directions. Information from the directional spectrum can be used for this splitting, see Atanassov et al. 1985.

\subsubsection{Parameter dependence and robustness}

It has been remarked already that the dimensionless quantity in the vertical direction that determines the effects of shadowing is the ratio of radar height and significant wave height: the larger this ratio, the less effect of shadowing at a fixed position. This has been confirmed for other study cases that will not be reported here. The dimensionless quantity in the horizontal direction is the ratio of distance to the radar and the peak wave length, and has the same consequence. The length of the maximal prediction interval in case of multi-modal sea states will depend in a somewhat complicated way on the relative energy contents and the difference of group speed of the wind waves and swell. For the study case described above (with 3 times larger significant wave height for the wind and with 2 times faster speed of 
the swell) the correlation as measure of quality seems to be too crude to identify the full effect of the swell; yet in observations of the spatial plots (or on cross sections) the difference can be noticed somewhat.

As is already indicated in Tables 2.2 and 2.3. almost irrespective the reconstruction of the shadowed seas, the DAES process produces substantially improved results in the near-radar area, with correlations between 0.88 and 0.95 depending on the reconstruction method. This robustness of the dynamic averaging and evolution scenario was also observed in other simulations. As an example, one other study case considered much wider spreading in the wind waves and swell. Although given by the same parameters as reported here, the argument $\theta-\theta_{\text {main }}$ in the spreading function was divided by 2 (which is sometimes also used). As a consequence, there is more overlap between the two sea states, and hence more counter propagating waves that will be evolved in the wrong direction. Nevertheless, correlations above 0.9 were obtained in the near radar area. A possible explanation for this seemingly inconsistent observation is that the much shorter waves cause less shadowing which may be a compensation in the measure given by the correlation.

\subsection{Conclusions and remarks}

In this paper we introduced a relatively simple and efficient simulation scenario to transform sequences of synthetic X-band radar images of multi-modal sea states into future sea states. The scenario turned out to be rather robust and produces reconstruction of the surface elevation in the blind area with correlation above 0.90 for the case of wind and wind-swell seas, for a ratio of radar height and significant wave height of 5. Additional simulations show that the correlation improves somewhat for higher values of this ratio because the effect of shadowing becomes less. No substantial differences are obtained for seas consisting of uni-modal wind waves or for multi-modal wind-swell seas.

The actual computation time for the simulation with the assimilation can run in real time; the required Fourier transforms for the averaging and evolution are executed within fractions of real time. For nonlinear simulations this may be somewhat longer but will not jeopardize the possibility to run the dynamic averaging-evolution scenario in real time. The dynamic averaging-evolution scenario providing updates for a running evolution can be used in other cases also when a dynamic system experiences perturbations. We close with mentioning some topics worth of further investigations and possible improvements.

The simplification to consider linear seas above constant depth in this paper is mainly for ease of presentation and execution of the scenario; nonlinear seas above topography could be dealt with straightforwardly. Apart from this, our understanding of waves in real seas still seems to be quite rudimentary. Even for linear waves, concepts as the main evolution direction introduced here have not yet been related to energy propagation direction; the MED for WS15 is remarkably different from the direction of the main energy carrying wind waves that determines the direction of the change of the wave profiles during evolution. Besides that, detailed studies of nonlinear seas may show phenomena that are not captured by linear seas, such 
as the occurrence and physical processes that lead to freak-like waves. If coherent interference is the main process for the appearance of long crested freak waves with relatively low Benjamin-Feir index, as indicated by Slunyaev et al. [2005], Gemmrich and Garrett 2008] and Latifah and van Groesen [2012], the same process may also lead to freak waves in short crested waves, enhanced by nonlinear interaction processes.

In the reconstruction process in this paper, we assumed the significant wave height of the sea to be given. Recent investigations showed that this information can actually also be extracted from the geometric images, see Wijaya and van Groesen 2016. Practical applicability requires the application of the full simulation scenario to real radar images and to test the results against accurate measurements. Another item to be clarified is if the accuracy of the predicted sea in the inner-radar area as achieved here, is sufficiently high to obtain accurately the forces on the ship carrying the radar, a topic of direct relevance for various practical applications. Finally, perturbations from heavy wind bursts may influence the results; it would be interesting and relevant to investigate the effects. 


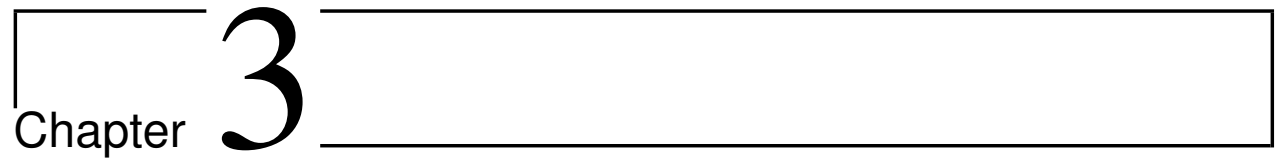

\section{Determination of the}

\section{significant wave height from shadowing in synthetic radar images ${ }^{1}$}

\section{Abstract}

Radar imagery is nowadays used to observe ocean waves despite the fact that radar images contain invisible areas because of the shadowing effect in the radar mechanism. Moreover, the radar images show the radar intensity which is not directly related to the wave height. This paper deals with the subject to estimate the significant wave height $H_{s}$ from (synthetic) radar images and will show that the change of shadowing with distance from the radar is the key property to estimate $H_{s}$. In fact, the extent of shadowing, quantified by a visibility function, depends on various physical variables, but most notably on two dimensionless quantities: the ratio of radar height and $H_{s}$ in the vertical direction, and the normalized distance in the horizontal direction. Assuming the normalized sea spectrum to be known, or approximating the spectrum, visibility functions can be determined for various $H_{s}$, defining the ingredients of a database. Comparing an observed visibility from successive images with the elements of the database, a best-fit approximation will provide an estimate of the $H_{s}$ of the observed sea. Randomness of the sea will only slightly affect the observed visibility and Monte Carlo simulations will annihilate these effects in the database.

\footnotetext{
${ }^{1}$ Published in this form except for references as:

A.P. Wijaya and E. van Groesen. Determination of the significant wave height from shadowing in synthetic radar images. Ocean Eng. 114, 204-215. 2016.
} 


\subsection{Introduction}

The use of radar images to monitor ocean waves can be beneficial for many offshore engineering applications to improve operability and safety. Sea parameters that can already be retrieved from the analysis of radar images include the peak period (e.g. Izquierdo et al. 2005]), wave current [Young et al., 1985], wind information Lund, 2012], and bathymetry Bell, 1999]. Although radar backscatter is not directly related to the wave heights, it is also desirable to determine the significant wave height $H_{s}$ as the parameter that characterizes the sea. Substantial research has been carried out to estimate $H_{s}$, either with or without calibration using in-situ measurements. Roughly speaking, two different methods have been used in the past to achieve that aim; one using the reconstruction spectrum and another using the spatial dependence of the shadowing phenomenon.

The most commonly used method in the spectrum-approach is to estimate $H_{s}$ from the so-called signal-to-noise ratios (SNR). It was introduced by Alpers and Hasselmann [1982 for synthetic aperture radar (SAR). The SNR was used to estimate the sea spectrum such that $H_{s}$ was calculate as four times the square root of the estimated spectrum area [Plant and Zurk, 1997]. For X-band radar, a 3DFFT method Young et al. 1985] was used to calculate the SNR as described in Borge et al. 1999. In contrast to Plant and Zurk [1997], $H_{s}$ was taken to be linearly related to the square root of the SNR with two free parameters which were calibrated from in-situ measurements. The other spectrum-approach used the so-called Doppler spectra to retrieve the surface elevations Johnson et al., 2009]. The result produced a standard deviation of error $1.07 \mathrm{~m}$ (for VV polarization) and $0.91 \mathrm{~m}$ (for $\mathrm{HH}$ polarization) of significant wave height $4.67 \mathrm{~m}$.

The other approach uses the distribution of shadowed areas that result because of geometrical shadowing, which is the effect that waves closer to radar can block the ray so that waves further away become invisible. In this respect, it should be remarked that in Plant and Farquharson 2012a it was argued that the geometric shadowing does not play a role in the radar mechanism; in contrast, partial shadowing is claimed to be the effect that appears in the images. The given explanation is that the diffraction of the electromagnetic signal causes a backscatter signal from the surface elevation that occupies the geometrical shadowed areas. However, the partial shadowing depends on the type of the polarization from the radar, and the difference with the geometrical shadowing may be very small.

Concerning the geometric shadowing, a statistical concept based on the proportion of the visible ('islands') and the invisible ('troughs') part of the waves was introduced by Wetzel [1990]. The probability of illumination $P_{0}$ was defined and related to $H_{s}$. In Buckley and Aler [1998a] it was shown that the estimation of $H_{s}$, using a constant $P_{0}$ that was calibrated from in-situ measurements, was only accurate for certain wave conditions, for instance when the ration of radar height and the wave height was high. An improvement was obtained by varying $P_{0}$ as shown in Buckley and Aler 1998b. A method without using any reference measurement, described in Gangeskar 2014], estimates $H_{s}$ from the RMS of the surface slope which is related to the shadowing effect. The relation is found from the best fit of the shadowing ratio, the proportion of the invisible points as a function of grazing 
angle, with the so-called Smith's function [Smith, 1967]. The results compared to measurement with a correlation of $87 \%$.

In Wijaya and van Groesen 2014], a method to estimate $H_{s}$ for long-crested waves based on the geometrical shadowing has been reported. The basic idea of the method is that the amount of shadowing is related to $H_{s}$. Formulations to measure the shadowing level were derived earlier by Wagner 1966 and Smith [1967], and compared to experiments described by Brokelman and Hagfors 1966. These formulations assumed that the joint probability density of heights and slope was uncorrelated. It was verified later by Bourlier et al. 2000 that the correlated joint probability density of heights and slope performed better than the uncorrelated one compared with the shadowing function that was determined numerically by generating the surface Brokelman and Hagfors, 1966].

This paper reports the extension of the method in Wijaya and van Groesen 2014 to short-crested waves. The advantage of the proposed method is that the external calibration (as in e.g. Borge et al. 1999] and Buckley and Aler [1998b]) is not required. The basic idea is as follows.

The shadowing effect will influence the visibility of waves, where visibility is defined as the probability that the surface elevation is visible at a certain position. This will depend on many parameters such as the distance from the radar $r$, the radar height $H_{r}$, and the properties that characterize the (irregular) sea: the significant wave height $H_{s}$, the (peak) wavelength $\lambda_{p}$ and the shape of the wave spectrum. Because of shadowing, the visibility will decrease for increasing distance. At a fixed position, it can be expected that the visibility decreases for increasing $H_{s}$. In this paper we will show that this is indeed the case provided one uses the two dimensionless quantities $h=\frac{H_{r}}{H_{s}}$ and $\rho=\frac{r}{\lambda_{p}}$ in the vertical and horizontal direction respectively; then the visibility as function of $\rho$ is indeed a rather robust indicator for $H_{s}$. This result then gives the possibility to determine $H_{s}$ from the measured visibility (that can be determined directly from the radar images) by comparing it with a visibility database which is obtained by calculating the visibility from simulations of shadowed seas for various values of $h$. The shadowed seas for the database can be constructed using a specified model spectrum or using the normalized spectrum that is obtained by applying 3DFFT on a reconstructed sea using the method described in Wijaya et al. 2015] (or Naaijen and Wijaya 2014] for additional tilt modulation). To improve efficiency, the database is constructed in the main wave direction only, using Monte Carlo simulations to reduce randomness of the seas. Then, detecting the visibility from the radar images of an observed sea, a best-fit approach with the curves in the visibility database leads to an estimate of $H_{s}$ of the observed sea. For two test cases, one for uni-modal wind waves and another for multi-modal wind-swell waves, it will be shown that $H_{s}$ can be estimated with an accuracy of approximately $98 \%$.

The radar images are synthesized based on the geometrical shadowing which is valid as a first order approach of the backscattering phenomenon. The other effects in the radar mechanism, such as tilt and hydrodynamic modulation, are not taken into account in this paper. This assumption is mainly for a simplified presentation, and may be sufficient since the tilt and hydrodynamic modulation have a minor impact on the imaging mechanism compared to the geometric shadowing at grazing incidence Borge et al. 2004. It should be noted that the results of this paper are 
obtained for 'idealized' cases, i.e. linear seas, that are not subject to local or global effects of wind. In a forthcoming paper we will study the influence of such effect on the shadowing.

This paper is organized as follows. In Section 3.2 the construction of the synthetic wave and the synthetic radar images is presented. The dimensionless variables and the visibility for harmonic and irregular waves are described in Section 3.3 . In Section 3.4 the method to estimate the significant wave height of a shadowed sea is described, and the result of several test cases will be presented in Section 3.5. Conclusions and remarks in Section 3.6 will end the paper.

\subsection{Synthetic Data}

The construction of the sea surface will be described in the first subsection. The resulting seas will be used to synthesize the radar images in the next subsection.

\subsubsection{Synthetic sea}

To model 2D water waves generated by winds, a directional wave spectrum $E_{2}(\omega, \theta)$ is frequently employed. It is obtained by multiplying a wave spectrum $E(\omega)$, e.g. a Jonswap spectrum, with a normalized directional spreading function $D(\alpha)$. The commonly used model for spreading function is

$$
D(\alpha)= \begin{cases}A \cos ^{2 s}\left(\alpha-\alpha_{0}\right) & , \text { for }\left|\alpha-\alpha_{0}\right| \leq \frac{\pi}{2} \\ 0 & , \text { for }\left|\alpha-\alpha_{0}\right|>\frac{\pi}{2}\end{cases}
$$

Here, $A$ is a normalization factor so that $\int D(\alpha) d \alpha=1, s$ is a natural number which controls the width of the spreading function, and $\alpha_{0}$ is the main propagation direction. The synthetic sea was previously often modeled using the double summation method because the component amplitudes were easily related to the directional components. However, this method produces seas that are neither ergodic nor spatially homogeneous because phase locking occurs when identical frequencies propagate in different directions as pointed out in Jefferys 1987. To overcome these problems, we use the single summation method that assigns one direction to one frequency as proposed in Jefferys [1987] and reviewed by Miles and Funke 1987]. Hence, for given model spectrum $E(\omega)$ with frequencies $\omega_{n}$ (selected equidistantly with spacing $\Delta \omega$ ), let $k_{n}$ be the corresponding wavenumbers according to the exact dispersion relation for linear waves. The direction $\psi_{n}$ at $\omega_{n}$ is drawn randomly with probability given by $D(\alpha)$, as proposed by Goda [2010], and random phases $\phi_{n}$ are selected uniformly in the interval $[0,2 \pi]$. Using polar coordinates $(r, \theta)$, the sea surface elevation is computed as

$$
\eta(r, \theta, t)=\sum_{n=1}^{N} \sqrt{2 E\left(\omega_{n}\right) \Delta \omega} \cos \left(k_{n} r \cos \left(\theta-\psi_{n}\right)-\omega_{n} t+\phi_{n}\right)
$$

Snapshots of the sea surface at multiples of the radar rotation time $\Delta t$ are then given by

$$
S_{n}(r, \theta)=\eta(r, \theta, n \Delta t), \quad n=0,1,2, \ldots
$$




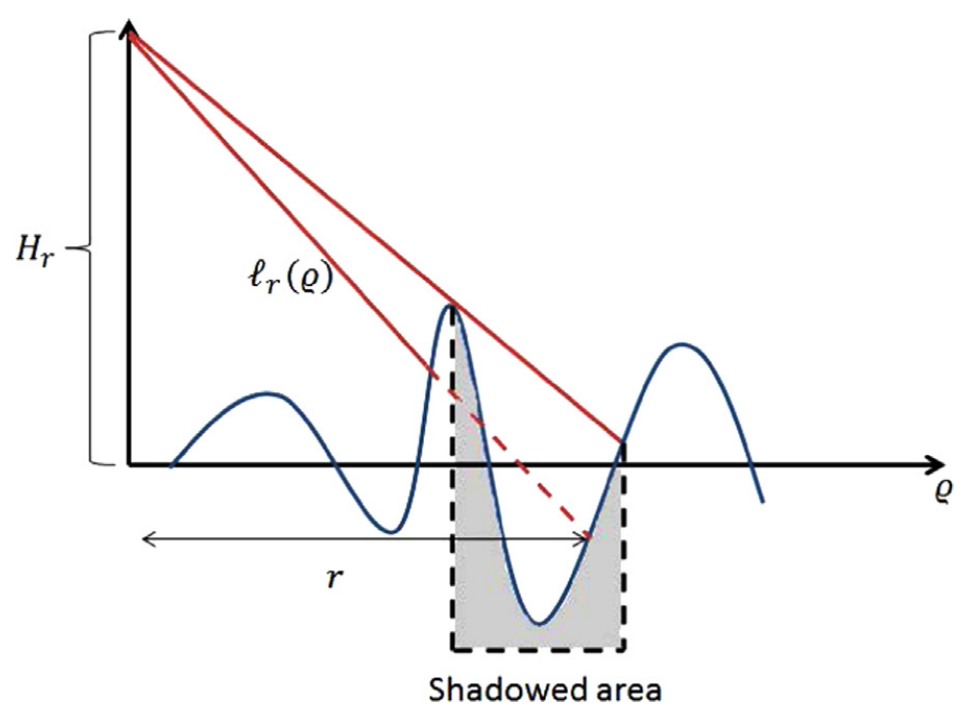

Figure 3.1: The elevation at $r$ is not visible by the radar because there are some points where $\ell_{r}(\varrho)$ is below the surface elevation in $0<\varrho<r$.

\subsubsection{Synthetic radar images}

Synthetic radar images are constructed by considering only the shadowing effect in the radar mechanism. Shadowing is a geometric effect caused by the fact that part of a wave may be invisible by the radar when it is blocked by waves closer to the radar; the shadowing effect will be more severe at far range than closer by the radar. Geometric shadowing is simulated on each ray so that the $2 \mathrm{D}$ problem reduces to many $1 \mathrm{D}$ problems. To describe it, let the radar be positioned at the origin and consider a radar ray in a direction with angle $\theta_{0}$. In that direction the surface elevation is given by $s(r)=S_{n}\left(r, \theta_{0}\right)$.

In Wijaya et al. 2015] the characteristic function of the visible points was defined: at given $r$, it depends on whether or not the elevation heights at points $\varrho$ closer to the radar are below the straight line $\ell_{r}(\varrho), 0<\varrho<r$ connecting the radar and the point $(r, s(r))$ (see Fig. 3.1):

$$
\chi(r)=1+\operatorname{sign}\left[\min _{\varrho}\left\{\Theta(r-\varrho) \Theta(\varrho)\left(\ell_{r}(\varrho)-s(\varrho)\right)\right\}\right]
$$

Here, $\Theta$ is the Heaviside function, equal to one for positive arguments and zero for negative arguments. The characteristic function identifies the shadowed and the non-shadowed intervals by value 0 and 1 , respectively. Hence, the shadowed sea in a direction $\theta_{0}$ is given by

$$
s^{s h a}(r)=s(r) \cdot \chi(r)
$$

Repeating the process over all look directions $\theta$ produces the shadowed sea $S_{n}^{s h a}(r, \theta)$. Since marine radar images are typically shaped like a disc with an area around the radar where the radar backscatter is too high due to specular scatter from the sea 
surface Skolnik, 1969] and/or due to the interaction from the ship's hull, we remove all the elevations over a certain radius $r_{i n}$ around the radar. At multiples of the radar rotation time, $n \cdot \Delta t$, a snapshot of a synthesized radar image is then given by

$$
I_{n}(r, \theta)=S_{n}^{s h a}(r, \theta) \Theta\left(r-r_{i n}\right)
$$

\subsection{Visibility}

We define a visibility function as the probability that the surface elevation at one position is visible by the radar Wijaya and van Groesen, 2014. Let $\chi_{i}(r, \theta)$ denote the characteristic function 3.4 at position $r$ along direction $\theta$ of image $i$. Then, the visibility function of $M$ radar images at position $(r, \theta)$ is defined as the average over $M$ images

$$
v i s(r, \theta)=\frac{1}{M} \sum_{i=1}^{M} \nu_{i}(r, \theta)
$$

To reduce the dependence on the parameters involved in the visibility function we use dimensionless variables described in Section 3.3.1. Using these variables will reduce the number of simulations used to determine a visibility database later on. The visibility for harmonic waves is presented in Section 3.3.2 it is derived analytically and gives us some idea about the properties of the visibility. For realistic irregular ocean waves the visibility will be obtained from a sequence of $M$ images using Eq. 3.7. Note that the visibility that is obtained in this way still contains some randomness of the random phases and directions of the specific sea under consideration. In the following we will need the visibility that is independent of the randomness, that will be called the Averaged Visibility (AV). AV will depend only on the physical parameters of the sea such as significant wave height $H_{s}$, peak period $T_{p}$, and specifics of the model spectrum, such as spreading factor $s$, peak enhancement factor $\gamma$ in the Jonswap spectrum. The elimination of the randomness in AV will be achieved using Monte Carlo simulation methods, as will be described in the last subsection.

\subsubsection{Dimensionless variables}

A simple geometric argument about the shadowing leads us to choose dimensionless variables Wijaya and van Groesen, 2014]. Consider a radar with altitude $H_{r}$ that scans the incoming harmonic waves with amplitude $A$ and wavelength $\lambda$. All of these parameters, and the distance $r$ determine the visibility. Because of the shadowing, the visibility will decrease for increasing distance. Hence, two quantities in the horizontal direction, $r$ and $\lambda$, encourage us to choose a dimensionless variable $\rho$ as the distance $r$ normalized by the wavelength $\lambda$. Since there are only two parameters in the vertical direction, $A$ and $H_{r}$, one dimensionless variable $h$ is defined as the ratio of radar height $H_{r}$ and the amplitude $A$. The dependency of the visibility on the ratio $h$ is illustrated in Fig. 3.2 a. It shows the shadowed harmonic waves of amplitude $1 \mathrm{~m}$ and $2 \mathrm{~m}$ by radars (at $\rho=0$ ) with altitude $5 \mathrm{~m}$ and $10 \mathrm{~m}$ respectively at one instant. The waves have the same phase which leads to the same characteristic 

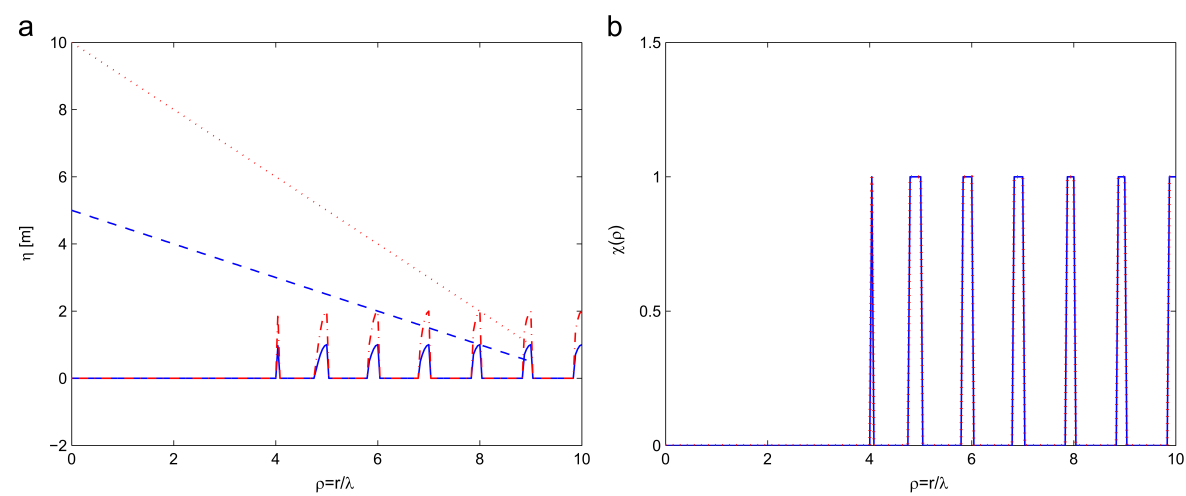

Figure 3.2: (a) The shadowing of harmonic waves of amplitude $1 \mathrm{~m}$ (blue, solid) and $2 \mathrm{~m}$ (red, dash-dotted) by radars (at $\rho=0$ ) of height $5 \mathrm{~m}$ and $10 \mathrm{~m}$ respectively. The critical rays from the $5 \mathrm{~m}$ (blue, dashed) and $10 \mathrm{~m}$ (red, dotted) radar scan the elevations at $\rho=8$. (b) The corresponding characteristic functions for the $1 \mathrm{~m}$ (blue, solid) and $2 \mathrm{~m}$ (red, dotted) harmonic waves are the same.

function $\chi$ as depicted in Fig. $3.2 \mathrm{~b}$. Applying the shadowing on the waves at different times will produce the same visibility (Eq. 3.7): a different position of the crest will change the characteristic function, but its effect in the visibility is ruled out because of the averaging as described in Eq. 3.7.

For irregular waves we adjust the dimensionless variables as $h=H_{r} / H_{s}$ and $\rho=r / \lambda_{p}$ where $H_{s}$ and $\lambda_{p}$ are the significant wave height and peak wave length, respectively. The dependence of the visibility on the dimensionless variables will be proved in the next subsection for both harmonic waves and will be shown from simulations for irregular waves.

\subsubsection{Visibility of long crested harmonic waves}

The derivation of the visibility function for harmonic waves is carried out for each ray. For harmonic long crested waves, the cross section on each ray is again a $1 \mathrm{D}$ harmonic wave with the same amplitude but with wave length that depends on the look direction. The visibility at a fixed position can be derived by determining the percentage of the wavelength that the waves are visible during the shifting over one wave length. Consider part of the wave in between two successive crests of which we call the crest near the radar as the first crest while the other as the second crest. We define $\xi_{l}$ as the (small) distance from the first crest to the point where the critical radar ray is tangent to wave slope, and $\xi_{r}$ as the distance from the second crest to the intersection point between the wave with the critical ray (see Fig. 3.3.

The visibility at distance $r$ for harmonic waves with amplitude $A$ and wave length $\lambda$ is then expressed by (see Appendix)

$$
v_{i s_{h a r}}(r)= \begin{cases}\frac{\xi_{l}(r)+\xi_{r}(r)}{\lambda}, & \text { if } r>\frac{\lambda H_{r}}{2 \pi A} \\ 1, & \text { if } r \leq \frac{\lambda H_{r}}{2 \pi A}\end{cases}
$$




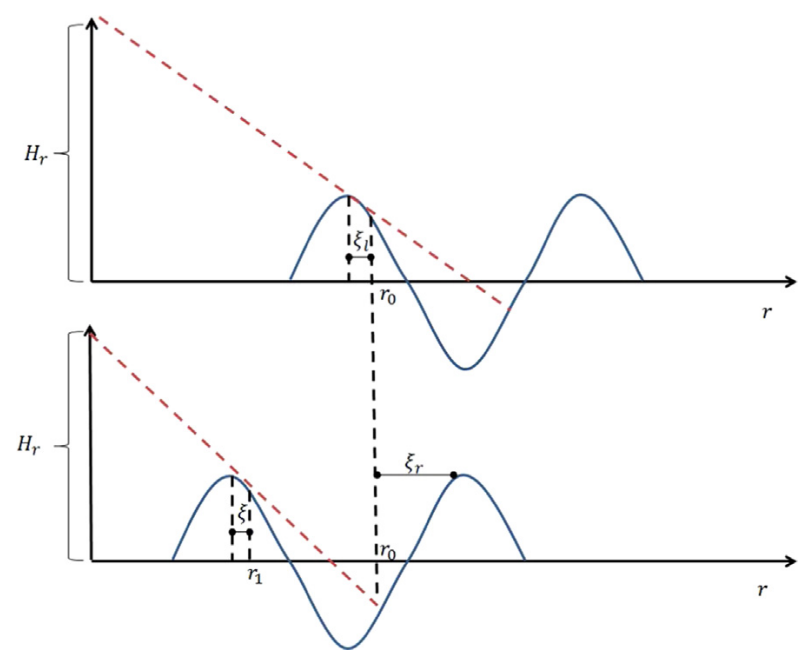

Figure 3.3: The extreme shadowing characteristics for a harmonic profile by a radar at height $H_{r}$. The top plot is the configuration to find $\xi_{l}$ whereas $\xi_{r}$ can be derived from the bottom plot that results after shifting the profile of the top figure.

Fig. 3.4 shows the visibility of harmonic waves with a period of 9 seconds above 50 $\mathrm{m}$ depth for different values $h=5$ and $h=10$ using Eq. 3.7 and the exact result of Eq. 3.8. To produce the result of Eq. 3.7 we used $M=1400$ images.

\subsubsection{Visibility of irregular waves}

For the uni-modal sea that will be used in Section 3.5 as one of the test cases, that propagates from the North, the visibility for case $h=5$ is shown by way of example in Fig. 3.5. Observe that, besides the dependence on the distance from the radar, the visibility also depends on the angle look direction. Fig. 3.5 a shows that, as expected, the lowest visibility occurs near the Northward direction, which corresponds to the opposite direction of the wind waves; the highest visibility is in directions almost perpendicular to the Northward direction. We will call the direction for which the lowest visibility occurs the Minimal Visibility Direction (MiViDi). The way how to detect the MiViDi will be discussed in Section 3.4. The visibility along rays in MiViDi and $45^{\circ}$ to the right of MiViDi are shown in Fig. 3.5 . We observe that these visibility curves are rather wiggly due to the randomness of the sea, even when 720 images are used as in Fig. 3.5. Removing the randomness will make the dependence of the visibility on the physical sea parameters more clear. The averaged visibility AV, that should be independent of randomness, is approximated by performing Monte Carlo simulations. The various steps in this procedure can be summarized as follows.

1. Given a frequency spectrum and a directional spreading function, construct the sea surface elevations using Eq. 3.2 with a random set of phases and directions. 


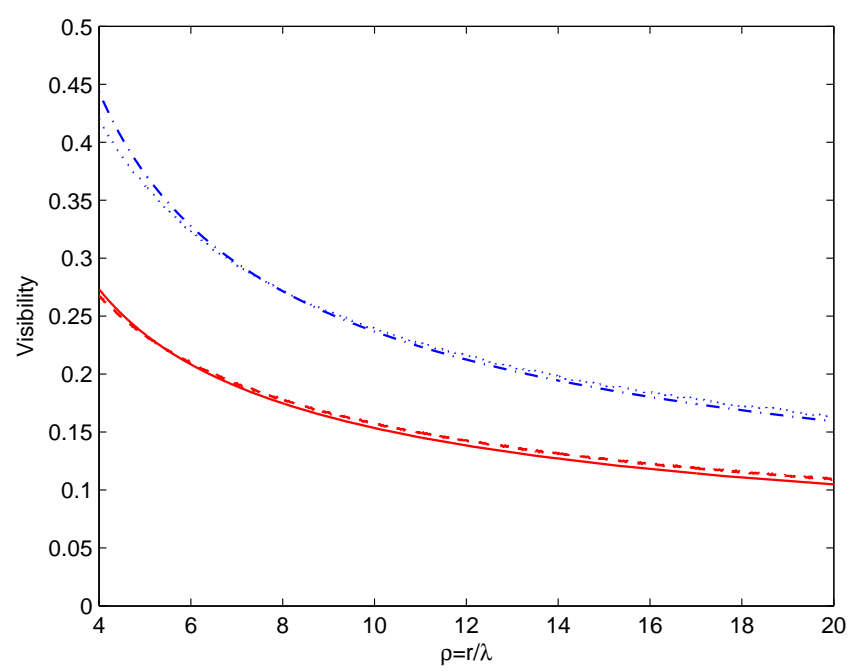

Figure 3.4: The visibility of harmonic waves for $h=5$ (red, solid) and $h=10$ (blue, dash-dotted) calculated by using Eq. 3.7. The exact results of Eq. 3.8 for $h=5$ and $h=10$ are indicated by red dashed and blue dotted line respectively. See the text for the used parameters.

2. Given the radar height $H_{r}$, apply the shadowing process.

3. Compute the visibility function from the synthetic radar images using Eq. 3.7 .

4. Repeat the process from step 1 to 3 a number $m$ of times for newly determined set of random variables, to produce $m$ visibility functions.

5. The AV is obtained by taking the average of the $m$ visibility functions.

To study the dependence of AV on the physical parameters, we consider variations of the parameters of the uni-modal sea described in Section 5, and show the results in Fig. 3.6. Each subplot shows the visibility as function of normalized radar distance in the direction of the MiViDi, the Northward direction. The AVs are obtained by averaging over $m=100$ visibility functions. Since the images have a blind area of radius $r_{i n}$ indicated by the region $\rho<4$ for the case described in Section 3.5, the results are plotted for $\rho \geq 4$. The different subplots indicate how the AV changes for changes in one of the various physical parameters. This dependence will be used essentially in the next section to detect the unknown wave height of a given sea spectrum. The dependence on the various parameters will now be described.

1. Ratio $h=H_{r} / H_{s}$

The shadowing increases for increasing $H_{s}$ at given radar height, corresponding to smaller values of $h$. This is shown in Fig. 3.6 for seas with fixed values of the peak period $T_{p}=9$, spreading factor $s=10$, peak enhancement factor in the Jonswap spectrum $\gamma=3$, for various values $h=2,6,10,14,18$. All 


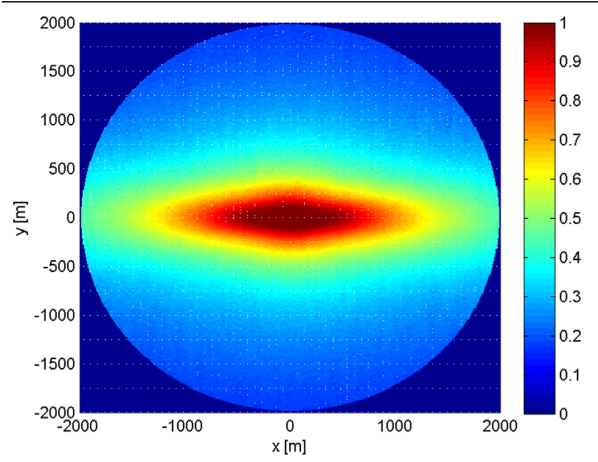

(a)

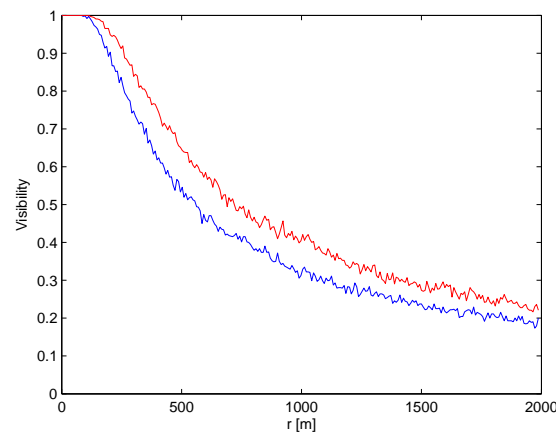

(b)

Figure 3.5: The visibility of $M=720$ synthetic radar images for a specific uni-modal sea from North to South described in Section 3.5 with ratio $h=5$. In (a) for all look directions and (b) in the direction of MiViDi (in this case at the Northward direction, blue solid line) and at $45^{\circ}$ to the right of MiViDi (red dashed line).

plots drawn by solid lines in the other subplots in Fig. 3.6 have these same parameters.

2. Peak period $T_{p}$

Fig. 3.6 b shows that for higher period the AV is smaller; the AV curves for higher period are depicted by dashed lines. The other parameters are the same as for the solid line.

3. Spreading factor $s$

The smaller the spreading factor $s$, the shorter the crest will be, which will give a better visibility far away. As a result, the shadowing is less severe for smaller $s$ as can be observed in Fig. 3.6. The differences are larger for higher $h$.

4. Peak enhancement factor $\gamma$ in Jonswap spectrum

The peak enhancement $\gamma$ has only a slight effect on the AV for small $h$; Fig. $3.6]$ shows the comparison of the AV for $\gamma=1,2,3$.

\subsection{Hs estimation method}

In this section we describe a method to estimate the significant wave height from a sequence of synthetic radar images. The idea of the method is based on the observation that the AV depends strongly on the significant wave height $H_{s}$, i.e. on the ratio $h$. Hence, if the normalized spectrum, or some physical parameters of the observed normalized sea spectrum, are known, we are able to construct the AV for various ratios $h$. Then, from the images of the observed sea, the visibility will be calculated, after which the AVs will be used as a database with which the visibility of the observed sea can be compared and the ratio $h$ of the observed sea can be 


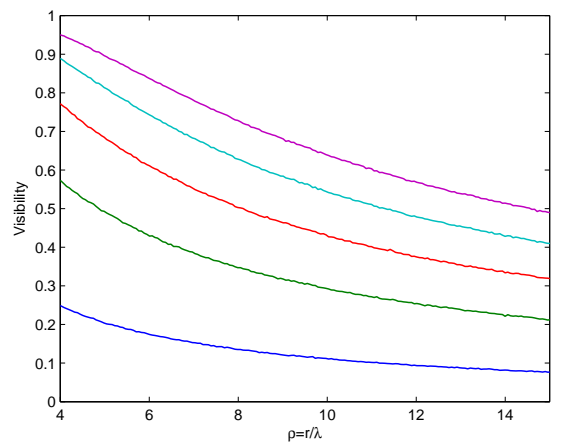

(a) Solid: $T_{p}=9, s=10, \gamma=3$

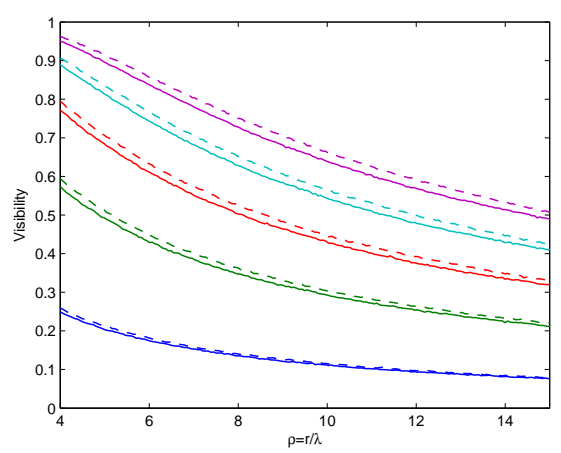

(c) Dashed: $s=2$

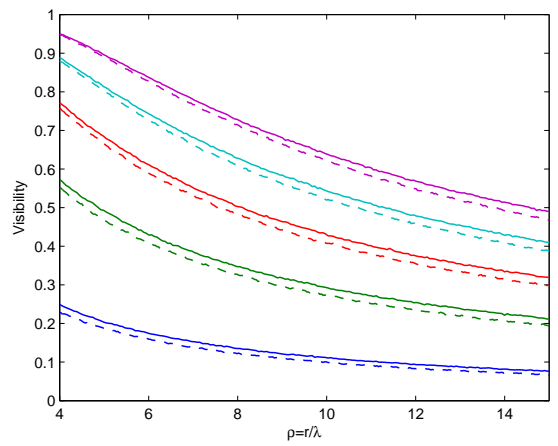

(b) Dashed: $T_{p}=12$

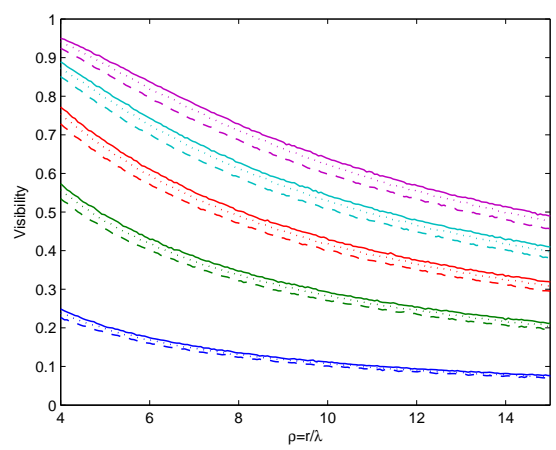

(d) $\gamma=1,2,3$ (dashed, dotted, solid)

Figure 3.6: Comparison of the Averaged Visibility for various parameters in the direction of MiViDi. The colors indicate the various ratios $h$ : blue, green, red, cyan, violet correspond to the ratio $h=2,6,10,14,18$ respectively. The $A V$ for different $h$ values, and the default parameter settings are shown in (a). These solid lines are copied to the other subplots in which dashed lines indicate the $A V$ when the value of $T_{p}, s$, or $\gamma$ are changed (compared to (a)) in (b), (c), and (d) respectively. 
determined. Knowing the radar height $H_{r}$ in advance, the significant wave height $H_{s}$ is obtained from $h=H_{r} / H_{s}$.

Since a $2 \mathrm{D}$ visibility database requires substantial memory and computation time mainly in the construction of the full circular short-crested seas, we design a $1 \mathrm{D}$ database that corresponds to a cross section of the $2 \mathrm{D}$ visibility database in the direction of MiViDi. Hence, the short-crested seas needed for the database construction are generated only at the MiViDi direction. This can be done by calculating $\eta\left(r, \theta_{M V D}, t\right)$ in Eq. 3.2. Therefore this section will begin with the detection of the MiViDi, and then the way to construct the database in the next two subsections. The comparison of the visibility with the database will be done by curve fitting as described in the last subsection.

\subsubsection{Minimal Visibility Direction (MiViDi)}

The detection of the MiViDi is needed to construct the visibility database in that direction. Let vis $(r, \theta)$ be the visibility from a given sequence of radar images. Then, the corresponding direction MiViDi, $\theta_{M V D}$, can be obtained as the direction where the integral over distance of the squared visibility is minimal, i.e. the solution of the minimization problem

$$
\theta_{M V D} \in \min _{\theta}\|v i s(r, \theta)\|_{\left[r_{\text {in }}, r_{\text {out }}\right]}^{2}:=\min _{\theta} \int_{r_{\text {in }}}^{r_{\text {out }}} \operatorname{vis}^{2}(r, \theta) d r
$$

Here, $r_{i n}$ and $r_{\text {out }}$ are the inner and the outer radius of the radar images, respectively.

The MiViDi will vary somewhat for different simulations and for different number of images, caused by the randomness in the visibility. For a uni-modal sea, the MiViDi will be in the vicinity of the main direction of the waves; for a multi-modal sea it depends on the direction and energy density of the constituent waves.

\subsubsection{Design of database using model spectrum}

In this subsection we consider the case that the database is made using a model spectrum, for instance a Jonswap spectrum with directional function 3.1. Because the model spectrum will differ in general from the spectrum of an actually observed sea, we call this construction of a database a Model-Spectrum method. For given depth and given physical parameters the peak period $T_{p}$, the peak enhancement factor $\gamma$, the spreading factor $s$, and the main direction of the waves, we synthesize the normalized sea $\left(H_{s}=1\right)$. Since we only design the database in the direction of MiViDi, the sea surface elevations in that direction $\eta\left(r, \theta_{M V D}, t\right)$ are calculated using Eq. 3.2. The visibility database is obtained following the same procedure as described in Section 3.3.3 to construct the AV for several ratios $h_{i}$ averaging over 100 visibility functions. The averaged visibility from the database at the dimensionless position $\rho$ for ratio $h_{i}$ is denoted as $V\left(\rho, h_{i}\right)$.

\subsubsection{Design of database using an observed spectrum}

Instead of using a model spectrum, a database can also be designed using the normalized sea spectrum as it is retrieved from the radar images of an actually observed 
sea. An estimate of the directional sea spectrum from radar images was proposed using the 3DFFT method in Young et al. 1985. The estimated spectrum from the radar images might not be accurate because of shadowing. In this paper we calculate the normalized spectrum by applying 3DFFT from a deterministic reconstruction of the surface elevation from the radar images as described in Wijaya et al. 2015. The method produced the reconstructed sea with a correlation of approximately $95 \%$ in a circular area around the radar with $500 \mathrm{~m}$ radius for the severe case of $h=5$. The 3DFFT of a reconstructed sea state in a square area centered at the radar location yields a frequency-wavenumber spectrum $S_{3}\left(\omega, k_{x}, k_{y}\right)$. The 2D wavenumber spectrum $S_{2}\left(k_{x}, k_{y}\right)$ can be obtained by integrating $S_{3}\left(\omega, k_{x}, k_{y}\right)$ with respect to $\omega$ coordinate. By using this way the resulted spectrum is symmetric about $k_{x}=k_{y}=0$ and makes an ambiguity of the wave direction. In order to obtain the spectrum $S_{2}\left(k_{x}, k_{y}\right)$ with the wavenumber components representing the wave direction, $\omega>0$ is used in the integration of the spectrum $S_{3}\left(\omega, k_{x}, k_{y}\right)$ Young et al. 1985. The $2 \mathrm{D}$ frequency spectrum is then obtained as

$$
S_{2}(\omega, \theta)=S_{2}(k, \theta) \frac{d k}{d \omega}=S_{2}\left(k_{x}, k_{y}\right) k \frac{d k}{d \omega}
$$

with $k=\sqrt{k_{x}^{2}+k_{y}^{2}}$ and $\theta=\arctan \left(k_{y} / k_{x}\right)$. The $1 \mathrm{D}$ frequency spectrum $E(\omega)$ and the directional function $D(\theta)$ are then obtained as

$$
\begin{aligned}
& E(\omega)=\int_{0}^{2 \pi} S_{2}(\omega, \theta) d \theta \\
& D(\theta)=\int_{0}^{\omega_{\max }} S_{2}(\omega, \theta) d \omega
\end{aligned}
$$

From this information Eq. 3.2 can be used to synthesize the normalized sea. The database is then obtained by using the same procedure to construct the AV obtained by averaging over 100 visibility functions. Constructing a database using the estimated spectrum of the actual sea will be called the Observed-Spectrum method.

\subsubsection{Curve-fitting to estimate $H_{s}$}

To estimate the significant wave height from the $2 \mathrm{D}$ radar images, we use the same idea as described above and proposed already in Wijaya and van Groesen [2014] for the $1 \mathrm{D}$ case, namely that the $\mathrm{AV}$ for given $h$ determines the visibility. And reversely, an observed visibility can be compared with the database that has been constructed with sufficiently realistic values of the other physical parameters. The visibility curve from the observed sea can then be fitted into the database and a 'best match' for varying $h$ determines the approximate $H_{s}$. Linear combination of consecutive curves in the database $\beta V\left(\rho, h_{i}\right)+(1-\beta) V\left(\rho, h_{i+1}\right), 0 \leq \beta \leq 1, \forall i$ will be used to fit the visibility curve with $\beta$ and index $i$ the quantities to be optimized.

The practical fact that randomness is involved in the determination of MiViDi makes a single estimation less reliable. To overcome this problem, in the fitting procedure, we consider the visibility curve in a small sector deviating less than $10^{\circ}$ from the direction of MiViDi. As described in Section 3.3.3. the visibility in other 
look direction needs a scaling of the wave length. Therefore we add one variable in the optimization process, denoted as $a$, which makes it possible that the visibility in different look directions has the correct scaling of the wave length. Taken together, the curve fitting between the visibility and the database is a minimization problem over 3 variables: $\beta, i, a$. In a look direction $\theta$, this can be expressed as

$$
\left\{\hat{a}_{\theta}, \hat{\beta}_{\theta}, \hat{I}_{\theta}\right\} \in \min _{a, \beta, i}\left\|\beta V\left(\rho, h_{i}\right)+(1-\beta) V\left(\rho, h_{i+1}\right)-v i s(a \cdot \rho, \theta)\right\|_{I}^{2}
$$

Here, $\|f(x)\|_{I}^{2}:=\int_{\min I}^{\max I} f(x)^{2} d x$ and $I$ is the largest interval of the intersection between $\rho$ and $a \cdot \rho$. The estimated ratio, $h_{\theta}$, at look direction $\theta$ is given by

$$
h_{\theta}=\hat{\beta}_{\theta}\left(h_{\hat{I}_{\theta}}-h_{\hat{I}_{\theta}+1}\right)+h_{\hat{I}_{\theta}+1}
$$

The ratio $h_{e s t}$ of the radar height and $H_{s}$ of the observed sea is then taken to be the average over the considered look directions:

$$
h_{e s t}=\operatorname{mean}\left\{h_{\theta}\right\} \text {, }
$$

leading to the estimate of the significant wave height of the observed sea as $H_{r} / h_{\text {est }}$.

\subsection{Case Studies}

In this section we report about the results of two study cases that were also considered in Wijaya et al. 2015]: wind waves only and a combination of wind and swell. We start to describe the numerical and sea parameters for the construction of the sea states, the visibility of the observed sea and the AV as a database. The results for the $H_{s}$ estimation for the study cases are presented in the last subsection.

\subsubsection{Preparation for the visibility}

\section{Numerical and sea parameters}

The seas are constructed in a full circular area with an outer radius $2000 \mathrm{~m}$ above a depth of $50 \mathrm{~m}$. We use spatial step $d r=7.5 \mathrm{~m}$ and $d \theta=0.3^{\circ}$. The time step $d t=2 \mathrm{~s}$ is used and corresponds to the radar rotation time as in Wijaya et al. 2015. We construct 720 images corresponding to a radar rotation time $d t=2 s$ at a time interval of 24 minutes. The radar is positioned at the origin $r=0$ with fixed height $H_{r}=15 \mathrm{~m}$ above the mean water level. The synthetic radar images are created having an inner radius of $500 \mathrm{~m}$ and an outer radius of $2000 \mathrm{~m}$.

We describe the sea parameters separately for wind waves and swell; the parameters for wind waves and swell are indicated by superscript $W$ and $S$ respectively. The wind waves propagate from North to South, the main direction is $\alpha_{0}^{W}=270^{\circ}$ (counter-clockwise from the positive $x$-axis), with spreading factor $s^{W}=10$. The spectrum of the wind waves is a Jonswap spectrum with peak enhancement factor $\gamma^{W}=3$, peak period $T_{p}^{W}=9 \mathrm{~s}$ and significant wave height $H_{s}^{W}=3 \mathrm{~m}$.

To construct the multi-modal sea, swell is added to the wind waves. The swell is from the South-East $\left(\alpha_{0}^{S}=135^{\circ}\right)$ with spreading factor $s^{S}=50$, and is constructed 
a

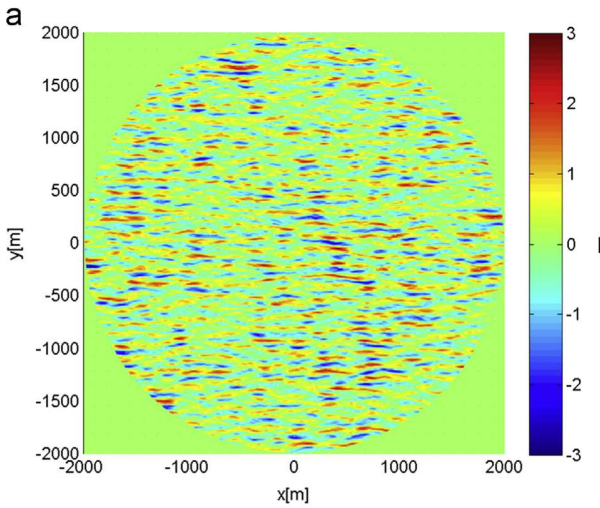

C

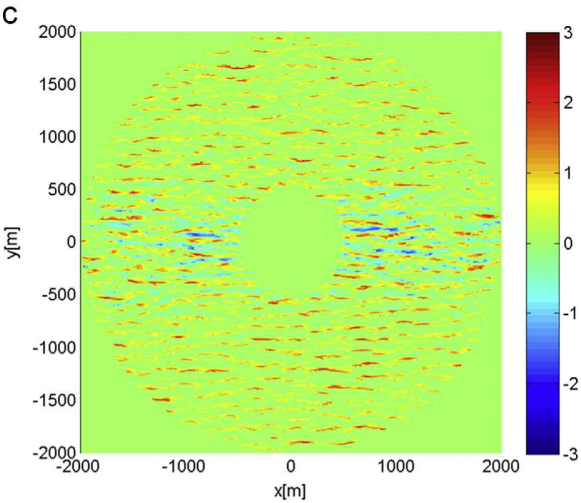

b

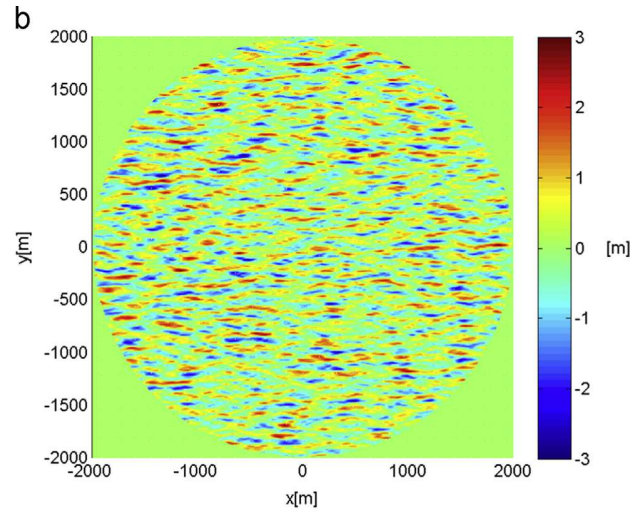

d

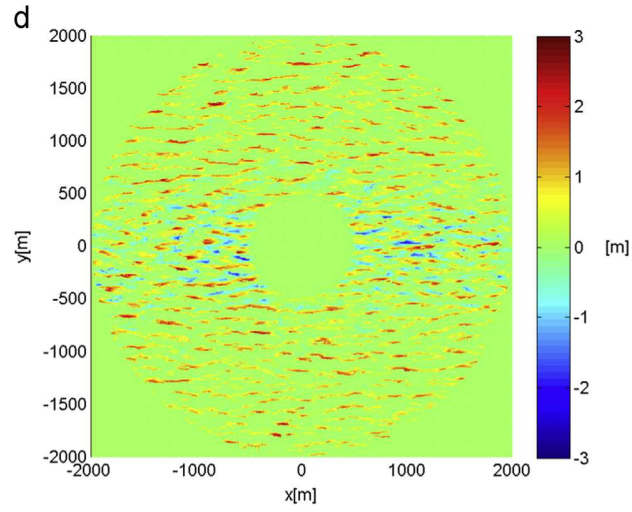

Figure 3.7: Images of the resulting synthetic data in the study cases. Image (a) shows the uni-modal sea propagating from the North, and (b) the multi-modal seas of wind waves from the North and swell from the South-East. Images (c) and (d) show the shadowed waves of image (a) and (b) respectively at the same instance. 


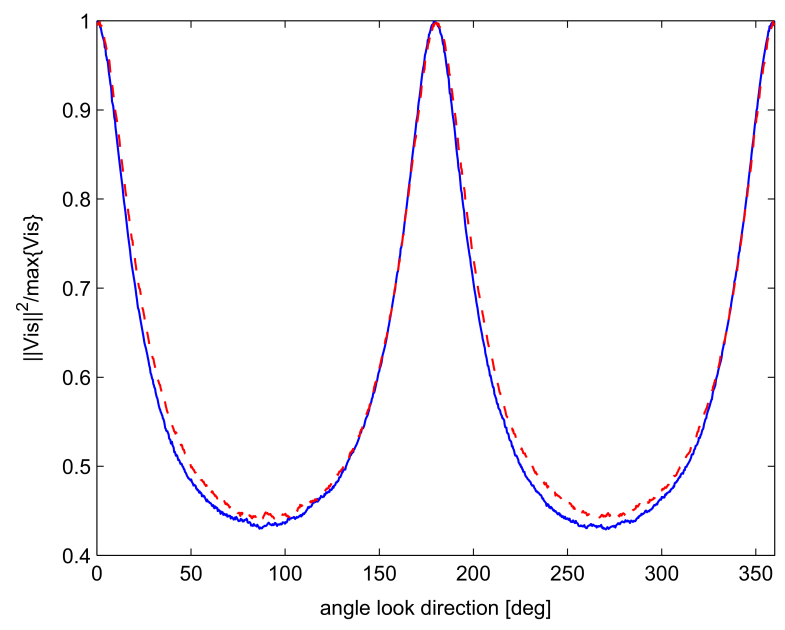

Figure 3.8: The normalized squared norm of the visibility over all angle look directions (Here, the angles are counterclockwise from the positive $x$-axis). The red dashed-line is for the uni-modal sea and the blue solid-line is for the multi-modal sea.

from a Jonswap spectrum with peak enhancement factor $\gamma^{S}=9$, peak period $T_{p}^{S}=$ $16 \mathrm{~s}$ and significant wave height $H_{s}^{S}=1 \mathrm{~m}$. As a result, the significant wave height for the multi-modal seas is $H_{s}=\sqrt{9+1} \approx 3.15 \mathrm{~m}$.

Fig. 3.7a shows the uni-modal wind sea and Fig. $3.7 \mathrm{~b}$ the multi-modal sea. Applying the shadowing effect as described in Section 3.2.2, yields the synthetic radar images as shown in Figs. 3.7p and $3.7 \mathrm{~d}$ respectively. From a sequence of the synthetic radar images for uni-modal seas the visibility functions in Fig. 3.5 were produced.

\section{Detection of the MiViDi direction}

After the synthetic radar images have been constructed, the visibility is calculated using Eq. 3.7. The MiViDi should be determined first before the curve fitting process is conducted. Fig. 3.8 shows the normalized squared norm of the visibility for the uni- and multi-modal cases over all angle look directions. This figure shows that the visibility is symmetric and there are two MiViDi values; we take the MiViDi in the upper half plane. For the uni- and multi-modal cases, the MiViDi is approximately $90.2^{\circ}$ and $90.9^{\circ}$ (averaged over 15 visibility functions) respectively; for both cases, the MiViDi is close to the opposite direction of the wind waves.

\subsubsection{Preparation of the visibility database}

The seas in the direction of the MiViDi $\eta\left(r, \theta_{M V D}, t\right)$ are calculated on $[0,2000] \mathrm{m}$ above the depth of $50 \mathrm{~m}$. We use spatial step $d r=7.5 \mathrm{~m}$, time step $d t=2 \mathrm{~s}$ and 24 minutes of time interval. The two ways to obtain the normalized sea spectrum as described in Sections 3.4 .2 and 3.4 .3 will be used to generate the visibility database. 

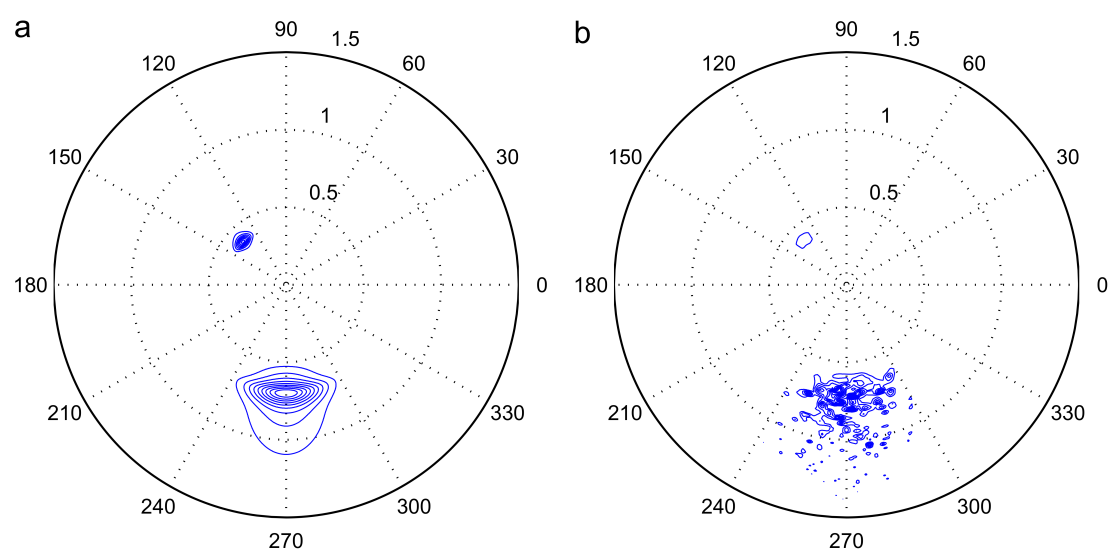

Figure 3.9: The 2D frequency normalized spectrum of (a) the Model- and (b) the ObservedSpectrum method for the multi-modal sea.

First, we use the Model-Spectrum method with the normalized sea spectrum, i.e. the Jonswap spectrum and the directional function as described in Eq. 3.1, are given with $H_{s}=1 \mathrm{~m}$ and with the other parameters that are the same as the parameters to generate the wind waves (for uni-modal case) and swell (for bi-modal case); see Fig. 3.10 for the shape of the wave spectrum.

Second, we use the Observed-Spectrum method. Using the method to reconstruct the sea as in Wijaya et al. [2015], the normalized sea spectrum is calculated using 3DFFT method applied on the reconstructed sea as described in Subsection 3.4.3. Since the waves are not fully recovered far away from the radar, the square area $[-1000,1000]^{2}$ of the reconstructed sea is taken to calculate the spectrum. Moreover, 256 images are used in the 3DFFT method.

The 2D frequency normalized spectra of the Model- and the Observed-Spectrum method for the multi-modal sea are shown in Figs. $3.9 \mathrm{a}$ and $3.9 \mathrm{~b}$ respectively. Although the shape of the observed spectrum looks very cluttered, the main direction of the wind waves and the swell can be captured very well. The quality of the spectrum reconstruction can be analyzed better by looking at the $1 \mathrm{D}$ spectrum. The model spectrum and the observed spectrum are shown in Fig. 3.10 . The observed spectrum yields a good approximation for the wave period and the main wave direction; $8.98 \mathrm{~s}$ and $15.5 \mathrm{~s}$ peak period of the wind waves and swell respectively, and $270^{\circ}$ and $135.6^{\circ}$ main direction of the wind waves and swell, respectively.

The spectrum of the reconstructed sea in the smaller area $[-500,500]^{2}$ is also given in Fig. 3.10. Although the reconstructed sea has a correlation of approximately $95 \%$ in the circular area of radius $500 \mathrm{~m}$ around the radar, the reconstructed spectrum has considerable deviations from the model spectrum. This is due to the fact that in that area, there are only a few waves which leads to a poor resolution in the Fourier analysis. This is also the reason why the swell spectrum is not estimated very well; the swell components are even fewer in the area $[-1000,1000]^{2}$. Since the shadowing effect led to a small but noticeable amount of energy in the low frequency 

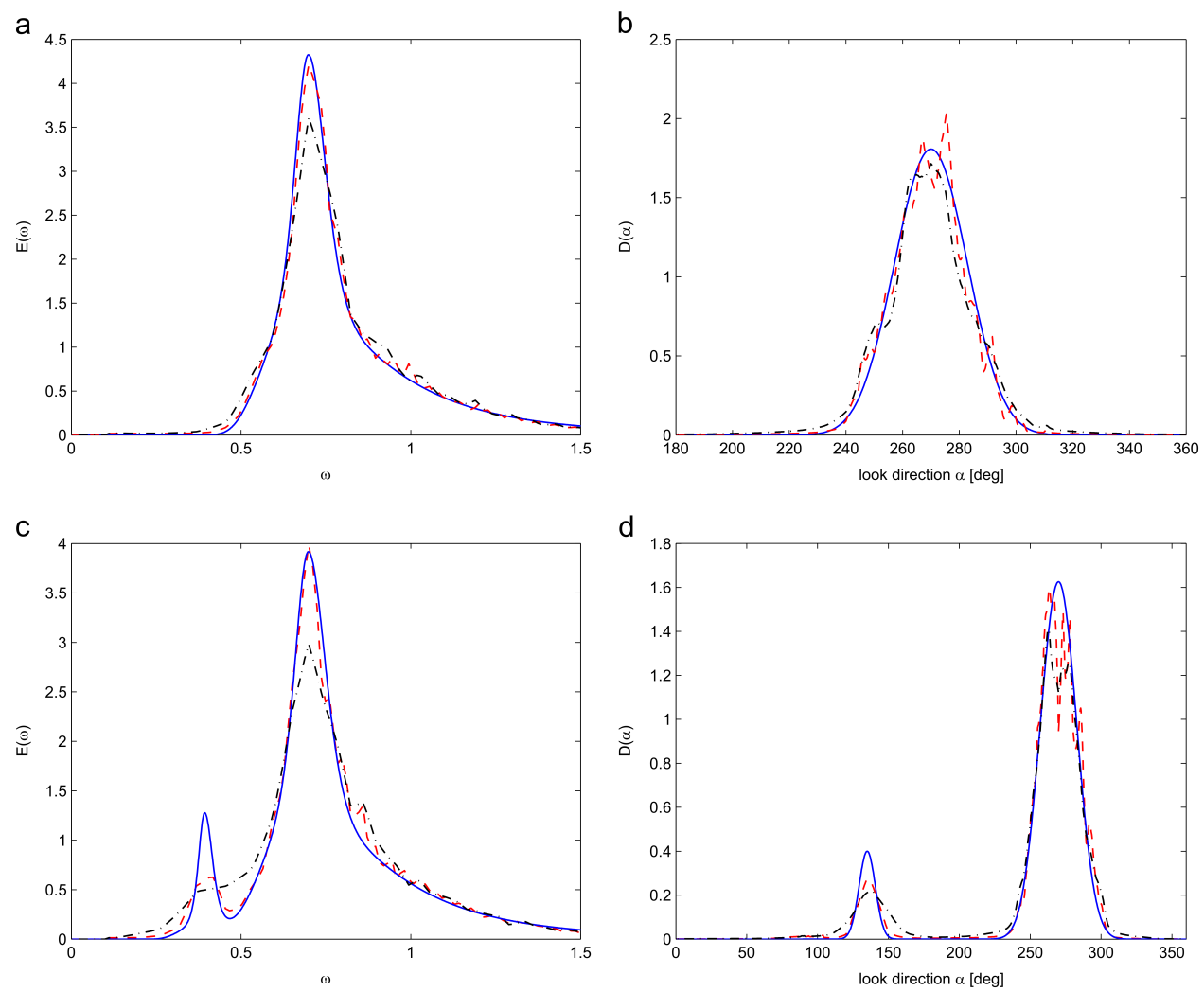

Figure 3.10: Ingredients of the model spectrum (solid blue), and the spectra of the reconstructed seas for a square area $[-1000,1000]^{2}$ (dashed red) and for area $[-500,500]^{2}$ (dashed-dotted black): the 1D frequency spectrum (left plots) and the directional spreading function (right plots) for the uni-modal (upper plots) and the multi-modal case (lower plots).

area, a high pass filter with cutoff frequency $\omega_{\text {cut }}=0.1$ has been applied. For both methods, for $h=4,6,8$, we averaged 100 visibility functions to obtain the AV as the database using the same procedure described in Section 3.3.3.

\subsubsection{Estimation of $H_{s}$}

In this subsection we present the result of the $H_{s}$ estimation for both study cases, using two different ways of constructing the database. Using the Model-Spectrum method and the Observed-Spectrum method to construct the database, the results for 50 realizations of the uni-modal sea are depicted in Fig 3.11 by the blue squared, and the red circular boxes respectively. The numerical results are listed in Table 3.1. For the Model-Spectrum and the Observed-Spectrum method the average value that estimates $H_{s}$ is 2.976 and 2.993, respectively; both have an average relative error below $1.05 \%$. 
Table 3.1: $H_{s}$ estimation results from 50 simulations for uni- and bi-modal sea indicated by letter ' $W$ ' and 'WS' respectively using Model- and Observed-Spectrum method.

\begin{tabular}{cccc}
\hline Data type \& method & Average $H_{s}$ & Standard deviation & Average error $(\%)$ \\
\hline W Model-Spectrum & 2.976 & 0.016 & 0.84 \\
W Observed-Spectrum & 2.993 & 0.038 & 1.05 \\
WS Model-Spectrum & 3.137 & 0.014 & 0.79 \\
WS Observed-Spectrum & 3.153 & 0.056 & 1.49 \\
\hline
\end{tabular}

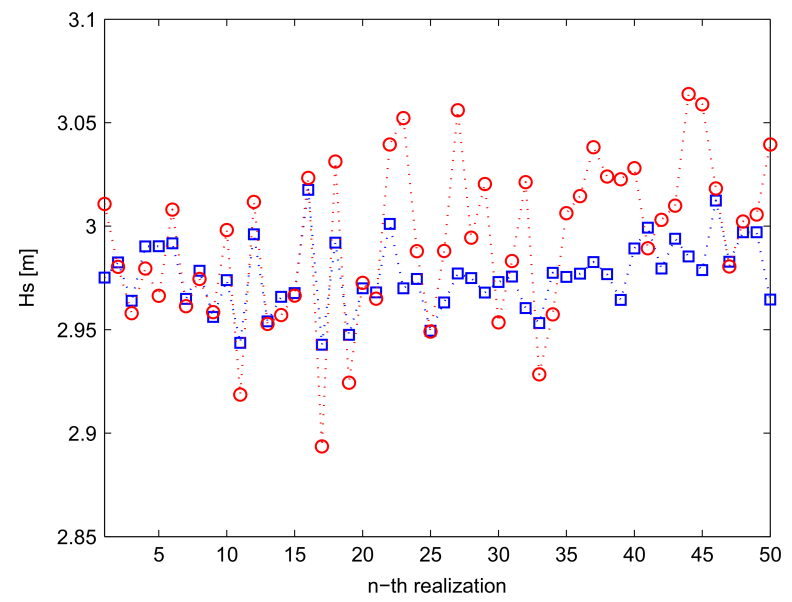

Figure 3.11: The estimated significant wave height over 50 realizations for the uni-modal sea; the blue squares give the results using the Model-Spectrum method to construct the database, the red circles using the Observed-Spectrum method.

In the same way, the results for the multi-modal sea are depicted in Fig. 3.12 and in Table 3.1. Now the estimates with the two methods for $H_{s}$ are 3.137 and 3.153 with average relative error of $0.79 \%$ and $1.49 \%$ for the Model-Spectrum and the Observed-Spectrum method, respectively.

In order to test the reliability of the proposed method we perform the $H_{s}$ estimation for other parameters. We consider wind-swell (WS) seas with parameters as stated in Subsection 3.5.1 except for one parameter that is changed: 1)depth $d=10 \mathrm{~m}, 2)$ spreading coefficient $s^{W}=5,3$ )parameter $\gamma^{W}=1$. As shown in Fig. 3.6, changing those parameters yields different visibility. We present results for the Observed-Spectrum method in Fig. 3.13. The mean (standard deviation; averaged error) of the estimated $H_{s}$ for $d=10, s^{W}=5$, and $\gamma^{W}=1$ are 3.099 $(0.024 ; 1.98 \%), 3.106(0.050 ; 2.03 \%)$, and $3.171(0.051 ; 1.27 \%)$ respectively. Note that the higher errors for case $d=10 \mathrm{~m}$ and $s^{W}=5$ are caused by the more complex cases to reconstruct the sea surface elevation by the DAES method; the shallower depth results in less visible waves due to the shorter wavelength and the smaller spreading factor makes the detection of the main evolution direction for bi-modal seas somewhat less accurate, see Wijaya et al. 2015]. 


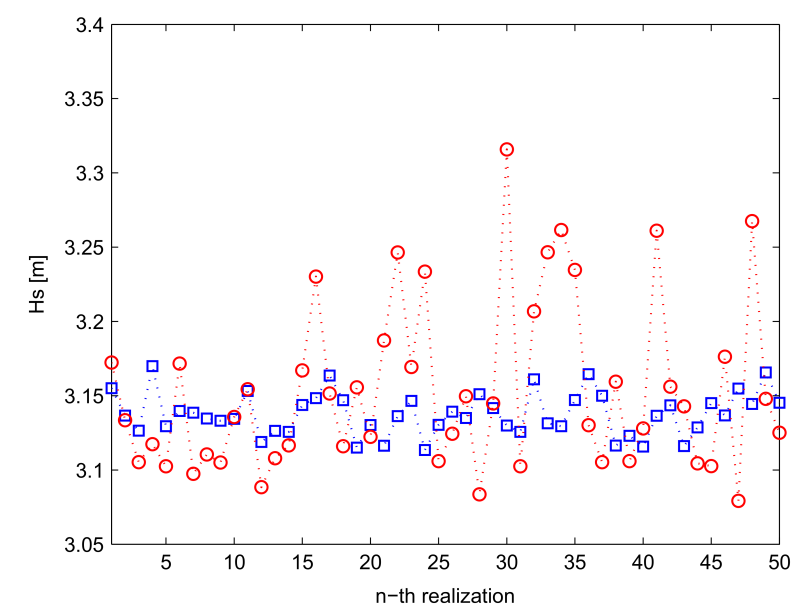

Figure 3.12: Same as Fig. 3.11 now for the multi-modal sea.

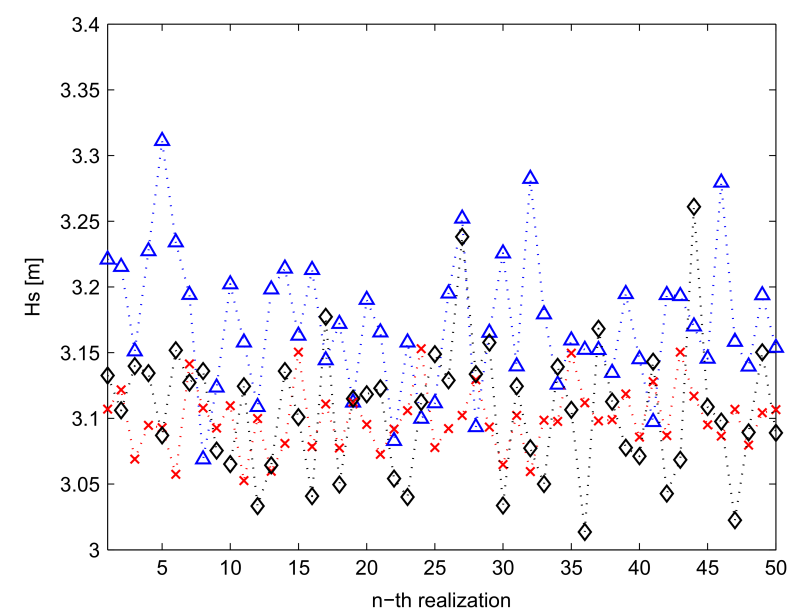

Figure 3.13: The estimated significant wave height over 50 realizations for the multimodal sea using the Observed-Spectrum method with changing one parameter; 1) $d=10$ (red crosses), 2) $s^{W}=5$ (black diamonds), and 3) $\gamma^{W}=1$ (blue triangles). 


\subsection{Conclusion and remarks}

The method to estimate the significant wave height described in this paper uses the difference of shadowing with distance. Essential for the success of the proposed method is the use of the dimensionless quantities in the horizontal and vertical direction. The test cases showed that $H_{s}$ can be found with very high accuracy. Below we will contemplate on two aspects for the practical usefulness of the methods: the application to real radar images including the effect of possible perturbations, and the time needed to perform simulations in operational conditions.

All the presented results are based on images that are obtained from a constructed synthetic linear sea using only the effect of shadowing into account. The question is if the proposed method is also useful when dealing with real radar images. In the introduction we already commented and gave references on the matter whether shadowing is the main effect observed in real radar images. Even if other effects, for instance partial shadowing, will determine the characteristics of the real images, the method can be adjusted. Using the property of distance dependence, and preparing a database based on the same mechanism as observed in the real images, the above described methods can be used. A different matter is if the assumptions made above that the seas can be constructed and evolved in a linear way, and that the effect of wind can be neglected, is a reasonable assumption. This will be left for further research in the study of nonlinear seas and the perturbing effects of especially local winds near the radar.

Concerning simulation times, we observed the following simulation times on a machine having a 8-core processor i7-3630QM CPU at $2.40 \mathrm{GHz}$. When using a (prepared) model-database, the only time needed is to calculate the visibility curve of the observed sea; from 720 images (correspond to 24 min sea-observation) this took approximately 1 min computation time. Producing the database from an observed spectrum, to reconstruct the images using the DAES method described in Wijaya et al. 2015] required approximately 8.5 min computation time, using 300 images (correspond to $10 \mathrm{~min}$ sea-observation). This information indicates that even in quickly changing sea states, an update of $H_{s}$ is almost instant, taking into account that an approximate $H_{s}$ will be known, so that only the AV in the database need to be constructed for neighboring values of the estimated value. Moreover, the number of the Monte Carlo simulations used in this paper seems too excessive for the practical purposes; already 10 to 20 Monte Carlo simulations are enough to produce acceptable results.

\section{Appendix}

The component of harmonic visibility $\xi_{l}$ and $\xi_{r}$

We consider a harmonic wave profile of amplitude $A$ and wavelength $\lambda: \eta(r)=$ $A \cos \left(\frac{2 \pi}{\lambda}\left(r-r_{0}+\xi_{l}\right)\right)$ (see Fig. 3.3. If $\xi_{l} \geq \frac{\lambda}{4}$, the harmonic wave will be visible over one wavelength. The length $\xi_{l}$ is obtained by solving the following equation

$$
\frac{2 \pi}{\lambda} r_{0} \sin \left(\frac{2 \pi}{\lambda} \xi_{l}\right)=\frac{H_{r}}{A}-\cos \left(\frac{2 \pi}{\lambda} \xi_{l}\right), \forall r_{0}>\frac{\lambda H_{r}}{2 \pi A}
$$


The critical value $r_{0}=\frac{\lambda H_{r}}{2 \pi A}$ is related to the length $\xi_{l}=\frac{\lambda}{4}$. The value of $\xi_{r}$ depends on the shift length $\xi$ (as depicted in the bottom plot of Figure 3.3). Two equations for $\xi$ and $\xi_{r}$ can be obtained by equalizing the slope at $r_{1}$ with the gradient of the radar ray connecting two pairs of different points $\left(r_{0}, r_{1}\right)$ and $\left(r_{0}, 0\right), \forall r_{0}>\frac{\lambda H_{r}}{2 \pi A}$, as follows

$$
\left\{\begin{array}{l}
-\frac{2 \pi}{\lambda} \sin \left(\frac{2 \pi}{\lambda} \xi\right)=\frac{\cos \left(\frac{2 \pi}{\lambda} \xi_{r}\right)-\cos \left(\frac{2 \pi}{\lambda} \xi\right)}{\lambda-\xi_{r}-\xi} \\
-\frac{2 \pi}{\lambda} \sin \left(\frac{2 \pi}{\lambda} \xi\right)=\frac{\cos \left(\frac{2 \pi}{\lambda} \xi_{r}\right)-H_{r} / A}{r_{0}}
\end{array}\right.
$$


$\Gamma_{\text {Chapter }}$

\section{Extensions of the DAES method}

This chapter presents an extension of DAES method to detect sea surface current in Section 4.1 and to reconstruct nonlinear waves (Draupner-like sea) from radar observations in Section 4.2.

\subsection{Sea Surface Current Detection ${ }^{1}$}

One important parameter in reconstructing and predicting the sea surface elevation from radar images is the surface current. The common method to derive the current is based on 3DFFT with which the (absolute) frequency is derived from a series of images and is fitted to the encounter dispersion relation that consist of the intrinsic exact dispersion relation for linear waves with an additional term that contains the current velocity to be found. The derived dispersion relation will be inaccurate because the images contain many inaccuracies from noise, shadowing, and other radar effects. This paper proposes an alternative method to determine the surface current. Following the method of the Dynamic Averaging and Evolution Scenario (DAES) as presented in Chapter 2, the idea is to choose the current velocity that minimizes the difference between an image at a previous time that has been evolved to the time of another image. In order to reduce inaccuracies, an averaging procedure over various images is applied. The method is tested on synthetic data to quantify the accuracy of the results. The robustness of the method will be investigated for several cases of different current parameters (speed and direction) for ensembles of seas with different peak frequency of characteristic sea states.

\footnotetext{
${ }^{1}$ The contents of the section is presented as a proceeding contribution Wijaya 2017
} 


\subsubsection{Introduction}

Sea surface current is one parameter of ocean waves that can be derived from radar images. The common method to retrieve the current is based on 3DFFT proposed in Young et al. 1985] where a least square method was used to fit an encounter frequency curve (the intrinsic frequency with an additional term that contains the current velocity) to the absolute frequency derived from the 3DFFT of images. Some improvements of the least square method have been made in Senet et al. [2001] by considering the nonlinear spectral structures and applying a spectral refolding technique. Moreover, an iterative algorithm was proposed in Senet et al. 2001] to update the velocity current estimation. In Gangeskar 2002], the 3D image spectrum was included as a weight function in the least square problem. Instead of the 3DFFT method, an alternative approach based on 2DFFT algorithm has been proposed in Abileah and Trizna 2010. The surface current and water depth were obtained by minimizing the difference between 2D Fourier components of image pairs. Another 2DFFT approach, called Conjugate Product method, was introduced in Alford et al. 2014 assuming that the phase shift of the conjugate product of two successive polar Fourier transform can be approximated as the absolute frequency times $\Delta t$ (the time difference between two consecutive images).

A different technique has been proposed in Serafino et al. 2010. The method is based on the maximization of the so called Normalized Scalar Product (NSP) between the filtered image spectrum and a characteristic function denoting a dispersion shell that depends on the unknown velocity current. The NSP method is able to detect relatively high speed currents. However, it needs a long computation time. In Huang et al. 2012], the method has been improved for both the computational efficiency and the precision by narrowing the variable search ranges iteratively.

Instead of comparing successive raw images as in Abileah and Trizna 2010], this paper proposes a current retrieval method that uses reconstructed images from DAES method presented in Chapter 2. The basic idea is to find the current velocity that minimizes the differences between images at previous times that have been evolved and averaged to the time of another image. The proposed method will be tested on synthetic data such that the accuracy of the results can be quantified. Synthetic radar images are constructed by taking into account the most dominant effect in the radar mechanism, i.e. the tilt and shadowing (Borge et al. 2004] and Naaijen and Wijaya 2014]). We assume that the radar is positioned on a fixed ship. Various current parameters for ensembles of sea states will be simulated to test the robustness of the method.

The structure of this section is as follows. Section 4.1.2 describes the construction of synthetic data. The details of the proposed current detection method is given in Section 4.1.3. Section 4.1.4 describes study cases and presents the results of the proposed method. Conclusions in Section 4.1.5 will finish this section. 


\subsubsection{Synthetic data}

\section{Synthetic Sea}

We generate a linear sea by superposing $N$ regular wave components. To achieve ergodicity properties of the sea we assign one direction to one frequency as proposed in Jefferys 1987. The sea surface elevations at time $t$ are computed in the polar coordinates $(r, \theta)$ as

$$
\eta(r, \theta, t)=\sum_{i=1}^{N} \sqrt{2 E\left(\omega_{i}\right) \Delta \omega} \cos \left(k_{i} r \cos \left(\theta-\alpha_{i}\right)-\omega_{i}^{e n} t+\phi_{i}\right)
$$

Here, $E(\omega)$ represents a wave frequency spectrum, $\Delta \omega$ is a constant spacing between the frequencies $\omega_{i}, k_{i}=\left|\mathbf{k}_{i}\right|$ are the wavenumbers corresponding to the frequencies $\omega_{i}$ via the exact dispersion relation for linear waves without current, $\alpha_{i}$ are the random directions drawn (as proposed in Goda [2010]) from the spreading function in Eq. 2.1. $\phi_{i}$ are phases selected randomly in the interval $[0,2 \pi]$, and $\omega^{e n}$ is the encounter frequency expressed by

$$
\omega^{e n}(\mathbf{k})=\sqrt{g|\mathbf{k}| \tanh (|\mathbf{k}| d)}+\mathbf{k} \cdot \mathbf{U}
$$

where $d$ is the water depth and $\mathbf{U}=\left(U_{x}, U_{y}\right)$ is the current velocity vector.

\section{Synthetic Radar Image}

The imaging of the ocean surface wave contains several modulations of radar backscatter. Three modulations have been reported to be the main source for the radar imaging: tilt, hydrodynamic modulation, and shadowing (e.g. Borge et al. [2004], Alpers et al. 1981, Dankert and Rosenthal 2004). In this paper the synthetic radar images are constructed by considering the most dominant effects in the marine radar mechanism, i.e. tilt and shadowing whereas the hydrodynamic modulation is assumed to have a minor contribution and will be neglected. The generation of the synthetic images will use the same procedure as in Naaijen and Wijaya 2014 for the tilt modulation and in Section 2.2.3 for the shadowing. We summarize the procedure as follows.

The tilt modulation is simulated by the scalar product $\sigma=\mathbf{u} \cdot \mathbf{n}$ where $\mathbf{u}$ is the $3 \mathrm{D}$ unit vector from the sea surface elevation to the radar antenna and $\mathbf{n}$ is the 3D unit normal vector of the sea surface elevation (e.g. Borge et al. [2004], Naaijen and Wijaya 2014), see Fig. 4.1. The shadowing effect creates areas where the radar can not sense the waves due to the obstruction by higher waves as also depicted in Fig. 4.1. The shadowed areas are assigned with the value 0 and define a characteristic function $\chi$ as in Eq. 2.3. The images at multiples of radar rotation time $\Delta t$ then are calculated as

$$
I(r, \theta, n \cdot \Delta t)=\sigma(r, \theta, n \cdot \Delta t) \cdot \chi(r, \theta, n \cdot \Delta t) \cdot \hat{\chi}_{r a d}(r)
$$

Here, $\hat{\chi}_{\text {rad }}(r)$ denotes the Heaviside function required to make a blind zone with radius $r_{i n}$ where there is no useful information as shown in typical marine radar images. 


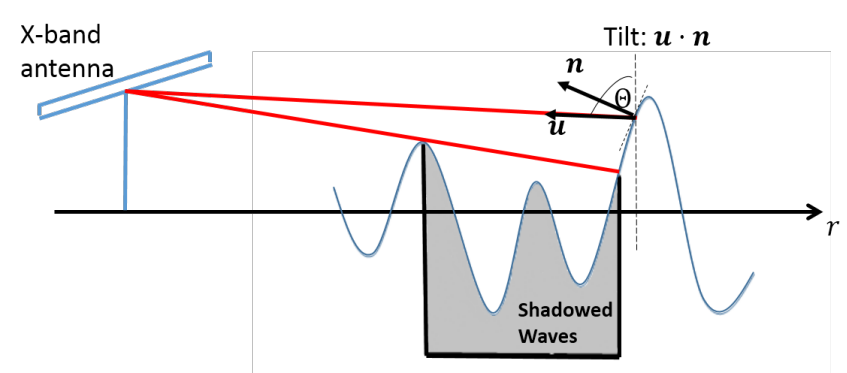

Figure 4.1: The tilt-shadowing mechanism. The shadowing effect results in invisible waves in the grey area. The tilt modulation is determined by the projection of the $3 D$ unit normal vector of the surface elevation $\boldsymbol{n}$ on the $3 D$ unit vector from the sea surface elevation to the radar antenna $\boldsymbol{u}$.

\subsubsection{Surface current detection}

Since the contribution of the surface current velocity appears in the encounter frequency in Eqn. (4.2), most current detection methods compare the dispersion model Eqn. 4.2 with the absolute frequency derived from images. This paper proposes a different method of which the surface current parameters are determined by comparing different physical images. For perfect images without any modulations, minimizing the difference of an evolved image at the later time of another image is sufficient to determine the current parameters. Since we are dealing with images that contain many inaccuracies the comparison by using only two images will not estimate the current velocity accurately. One way to improve the accuracy of the estimation is to include the reconstruction procedure, Dynamic Averaging and Evolution Scenario (DAES) as in Chapter 2. Starting with an initial current velocity $\mathbf{U}_{0}$, an updated current velocity is achieved such that the difference of the reconstructed image from DAES and the reference image is minimum. This can be expressed as

$$
\min _{\mathbf{U}}\left\|D A E S\left(\left\{R_{0}, R_{1} \ldots, R_{n}\right\}, \mathbf{U}\right)-R_{n}\right\|_{D}^{2}
$$

Here, $R_{i}$ denotes the vertical shifted image (such that the mean of $R_{i}$ is zero) at time $t_{i}=i(M+1) \cdot \Delta t$ where $M$ is the image sampling number chosen based on the sea period $T_{p}$. DAES $\left(\left\{R_{0}, \ldots, R_{n}\right\}, \mathbf{U}\right)$ is the result of the reconstruction method DAES from the image set $\left\{R_{0}, \ldots, R_{n}\right\}$ with the linear evolution that takes the current velocity $\mathbf{U}$ into account. The difference between the reconstructed image and the reference image $R_{n}$ is computed on a sub-domain $D$ which will be chosen as a rectangular area heading the main wave direction. To solve Eqn. 4.4 we use nonlinear least square method without any weight factor. The DAES method used in this paper is summarized as follows. We restrict to use three images as a basis of the DAES method. Here, we describe the image reconstruction process at time $t_{i}$. At that time, there are the image $R_{i}$ and the evolution of two images $R_{i-1}$ and $R_{i-2}$ over $(M+1) \Delta t$ and $2(M+1) \Delta t$ respectively. Each of them represents an approximated wave field with different information because the information at different time are 
distinct due to the shadowing. Averaging the data will contain more information and give a better approximation. Moreover, the evolution of the last reconstruction image at time $t_{i-3}$ over $3(M+1) \Delta t$ also represents an approximated wave field not only in the observation zone, but also in the blind zone. Therefore, with the same weight factors as in Eq. 2.12 the reconstruction image at time $t_{i}$ is calculated as

$$
\begin{aligned}
\eta_{i}^{r e c}= & \left(\frac{1}{6}\left(R_{i}+\varepsilon^{(M+1)}\left(R_{i-1}, \mathbf{U}\right)+\varepsilon^{2(M+1)}\left(R_{i-2}, \mathbf{U}\right)\right)+\frac{1}{2} \varepsilon^{3(M+1)}\left(\eta_{i-3}, \mathbf{U}\right)\right) \\
& \left(1-\hat{\chi}_{r a d}\right)+\varepsilon^{3(M+1)}\left(\eta_{i-3}^{r e c}, \mathbf{U}\right) \hat{\chi}_{\text {rad }}
\end{aligned}
$$

\subsubsection{Study cases and results}

We synthesize uni-modal seas above $50 \mathrm{~m}$ depth with Jonswap spectrum with significant wave height $H_{s}=3 \mathrm{~m}$ and peak enhancement factor $\gamma=3$. The waves are propagated to the South with spreading coefficient $s=10$. We simulate three different seas for peak periods $T_{p}=5,9,13 \mathrm{~s}$ that have phase velocity $7.8 ; 13.9 ; 17.8$ $\mathrm{m} / \mathrm{s}$ respectively. For each sea, 21 different current parameters are added by taking all combinations of

1. Three current speeds: $0.45 ; 0.9 ; 1.5 \mathrm{~m} / \mathrm{s}$

2. Seven current direction: $90^{\circ}, 120^{\circ}, 150^{\circ}, 180^{\circ}, 210^{\circ}, 240^{\circ}, 270^{\circ}$

Here, we use the reference $0^{\circ}$ pointing to the East with the counter-clockwise direction. The percentages of the current speeds with respect to the phase speed of each sea are shown in Table 4.1 .

Table 4.1: The percentages of the current speeds with respect to the phase speeds.

\begin{tabular}{cccc}
\hline & $T_{p}=5$ & $T_{p}=9$ & $T_{p}=13$ \\
\hline$|\mathbf{U}|=0.45$ & $5.7 \%$ & $3 \%$ & $2.5 \%$ \\
$|\mathbf{U}|=0.9$ & $11.5 \%$ & $6 \%$ & $5 \%$ \\
$|\mathbf{U}|=1.5$ & $19.2 \%$ & $10 \%$ & $8.4 \%$ \\
\hline
\end{tabular}

The seas are synthesized in a spatial polar grid with $d r=7.5 \mathrm{~m}$ and $d \theta=0.3^{\circ}$ within an outer ring of $r_{\max }=2000 \mathrm{~m}$. The synthetic radar images are constructed from a radar positioned at $r=0$ with $30 \mathrm{~m}$ height above the mean sea level. The radar rotation time is $\Delta t=1.5 \mathrm{~s}$ and the inner radius for the blind zone is $r_{i n}=500$ $\mathrm{m}$. An example of a synthesized image for the case of $T_{p}=9 \mathrm{~s}$ is shown in Fig. 4.2.

We perform 25 different image sets $\left\{R_{0}, \ldots, R_{n}\right\}$ that contain 28 images for each case. We sample the images every $\Delta t, 2 \Delta t, 3 \Delta t(M=0,1,2)$ for the case of $T_{p}=$ $5,9,13$ s respectively. We choose a sub-domain $D$ in Eqn. 4.4 as $x \in[-500,500]$ and $y \in[500,1000]$. The comparison between a reconstructed image (evolved with an estimated current parameter) and the reference image $R_{n}$ is shown in Fig. 4.3. It shows that the reconstructed image is in a good agreement with image $R_{n}$ at the visible points. The results of current estimation are presented in the polar plots Fig. 4.4 where the angle represents the current direction. The current estimations 


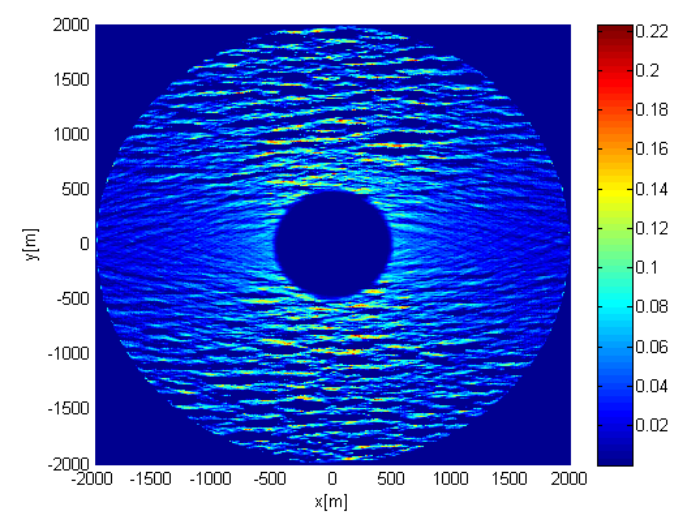

Figure 4.2: A tilt-shadowing image for the case $T_{p}=9 \mathrm{~s}$.

are shown in the first column where the radius represents the current speed. The current estimations converge for all the cases which indicate the robustness of the method. Note that the results for $|\mathbf{U}|=0.45$ in all $T_{p}$ cases are less accurate since the current speed is relatively low with respect to the phase speed, see Table. 4.1. This can be seen quantitatively by the averaged absolute error for the current direction and the averaged relative error for the current speed in the second and the third column respectively. Overall, the absolute errors of the current direction are below $10^{\circ}$, except for the case $|\mathbf{U}|=0.45$ in $T_{p}=5 \mathrm{~s}$. The relative errors of the current speed are below $10 \%$, except also for the case $|\mathbf{U}|=0.45$ in $T_{p}=5 \mathrm{~s}$ and $T_{p}=9 \mathrm{~s}$.

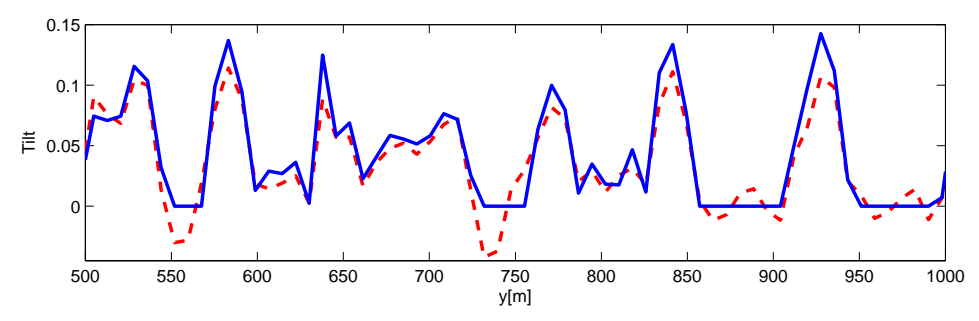

Figure 4.3: The cross section of a reconstructed image (red-dashed) evolved with an estimated current parameter and the reference image (blue-solid) along $x=0$ at interval $500<y<1000$.

\subsubsection{Conclusions}

A different approach to determine the surface current has been proposed in this paper. The method is based on the comparison of several images using the reconstruction method DAES to improve the accuracy of the surface current detection. The results show robustness and accuracy of the method for various current parameters. Moreover, the proposed method uses less parameters than in the spec- 


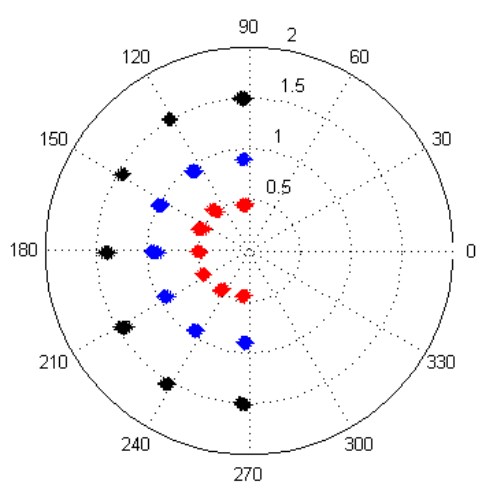

(a)

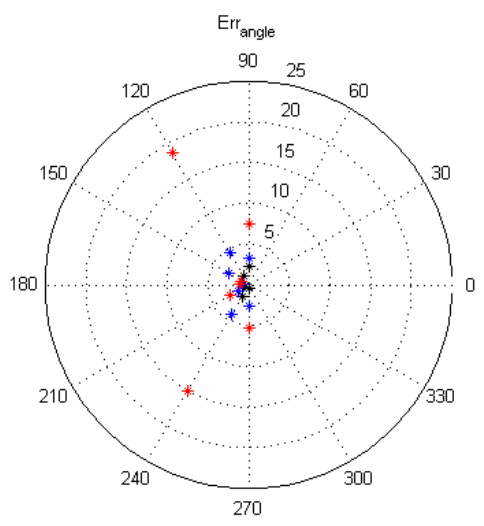

(b)

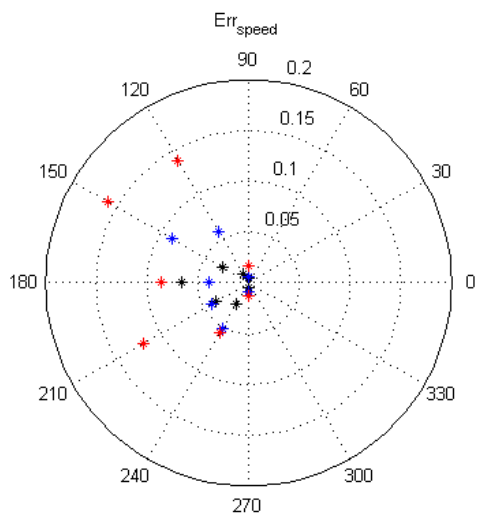

(c)

Figure 4.4: The current results for $T_{p}=5 \mathrm{~s}$. The red, blue, and black colors indicate the results for $|\boldsymbol{U}|=0.45,0.9,1.5$ respectively. The top left (a) represents the current estimations where the radius is the current speed, the top right (b) represents the averaged absolute errors of the current direction where the radius is in degrees, the bottom (c) represents the averaged relative errors of the current speed where the radius is dimensionless.

tral method Young et al., 1985], [Senet et al., 2001, Gangeskar, 2002, , Abileah and Trizna, 2010], [Alford et al., 2014], [Serafino et al., 2010], [Huang et al., 2012]. The only parameter that is varied for different periods $T_{p}$ is the sample image parameter $M$. It was found that for the optimal result the sampling image should be chosen such that the three images in the averaging process covering one wave period. The references that describe results of simulations to test their current detection method were presented in Senet et al. 2001] and Huang et al. 2012. The accuracy seems to be quite good, but unfortunately we can not compare fairly with the accuracy of our method since there was no indication how large is the current speed relative to the phase speed that they used. Some parameters needed to calculate the phase velocity are not specified: in Senet et al. 2001, the wave period and the depth are 


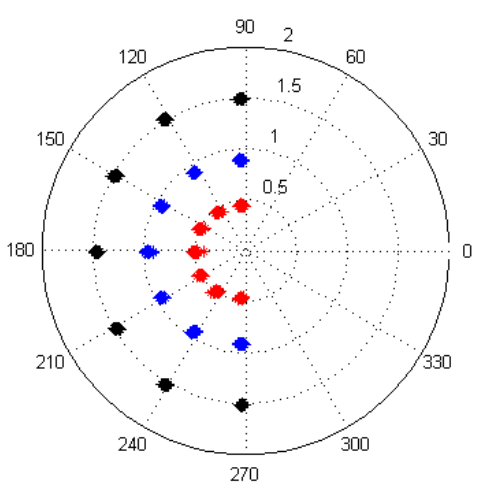

(a)

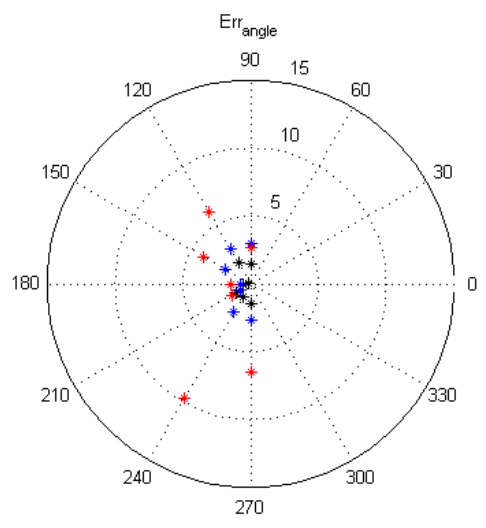

(b)

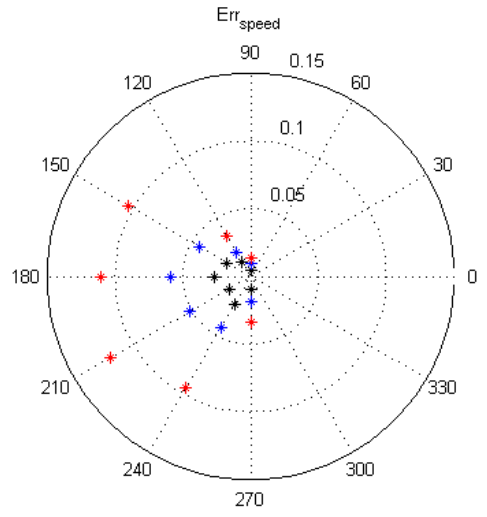

(c)

Figure 4.5: The same as Fig. 4.4, but for $T_{p}=9 \mathrm{~s}$.

not listed while in Huang et al. 2012], information about the depth is missing.

\subsection{Reconstruction and prediction of nonlinear waves $2^{2}$}

In this section the methods presented in Chapter 2 are extended to become applicable to high, Draupner-like seas. The main idea of the method is to reconstruct the sea states by improving the radar images by DAES, a dynamic averaging and evolution scenario. The procedure consists of data assimilation in an on-going evolution of the sea state; the assimilation is performed with updates that are obtained by dynamic averaging of successive images to enhance the quality of the updates. The evolution brings the waves from the ring-shaped radar-observation area into the blind area

\footnotetext{
${ }^{2}$ The contents of this section is an already improved version of part of a submitted paper van Groesen et al. 2017] slightly adapted to be more self-contained about the Draupner seas to be used.
} 


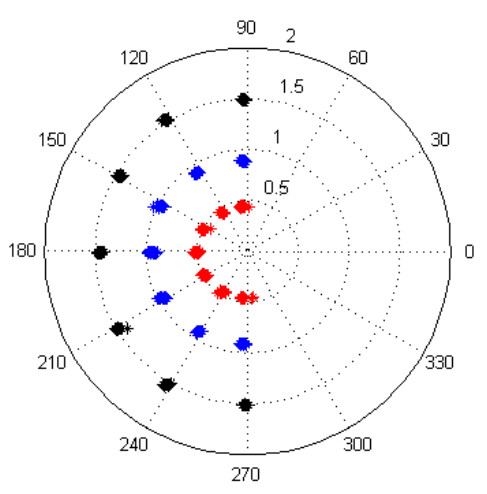

(a)

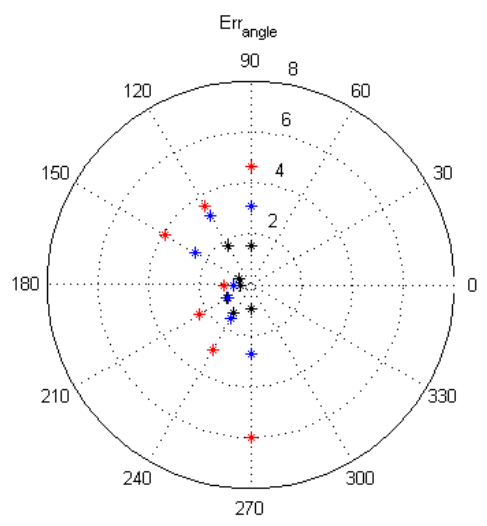

(b)

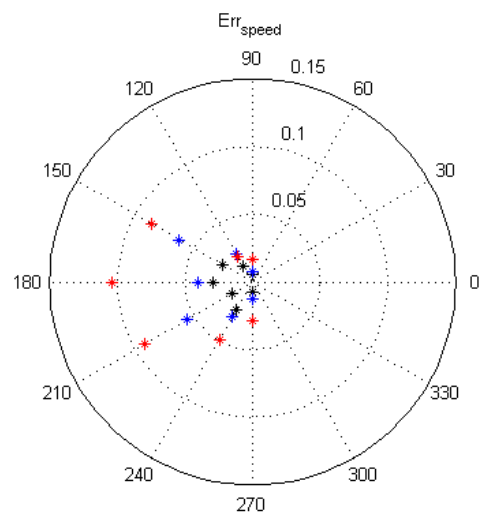

(c)

Figure 4.6: The same as Fig. 4.4. but for $T_{p}=13 \mathrm{~s}$.

surrounding the radar. The reconstruction works well for low, possibly multi-modal, seas with high correlations with the real sea state above $90 \%$.

Using one reconstructed sea state as initial value, this state can be evolved in time without any updates to produce a prediction of the expected sea in future time. Until the maximal physical prediction horizon, linear uni- and multi-modal sea states can be evolved towards the radar area with correlations above $90 \%$. In Chapter 3 it was shown that the shadowing effect actually also characterizes the significant wave height directly from the images without any empirical constants or further external information.

For low waves it is sufficient to use linear simulations in DAES with standard linear Fourier methods to advance the waves with the exact dispersion relation extremely fast. Here it will be shown that the same methods can be applied with good results for Draupner seas using the nonlinear AB-code to evolve the updated waves. Specifically, we will show the performance for a sea identified as number 13 from an ensemble of 40 seas (see van Groesen et al. 2017) for the area and time in 
which a freak-like wave train from north to south approaches the radar, for which a shift in coordinates is taken to have the origin in line with the direction of the train. A preliminary result for reconstructing nonlinear long-crested waves has been discussed in Wijaya 2016.

The first subsection describes the Draupner sea parameters that will be used to synthesize radar images. For details of the construction of the sea see van Groesen et al. 2017. In the second subsection (distorted) synthetic radar images are designed from the nonlinear sea states by adding the shadowing effects, and restricted to the radar observation domain to cover only a ring shaped area between 500 and 2000 $\mathrm{m}$ in the northern semicircle; the small semicircle with $r<500$ is the blind area of the radar where the radar backscatter is too high to be useful due to specular scatter from the sea surface [Skolnik, 1969]. After that a sort description is given of the idea to improve the quality of the images by an averaging technique. Using the wave elevations in the radar-observation area as updates, an ongoing dynamic evolution over the semicircle will translate the waves into the blind area towards the radar area. In the third subsection the prediction of waves ahead of time at the radar position will be considered and related to the maximal prediction horizon. Moreover, a possibility to detect a freak wave up to $150 \mathrm{~s}$ in advance is presented. In the last subsection the results for this demanding case will be discussed.

\subsubsection{Parameters of synthetic Draupner sea}

This subsection describes briefly the Draupner sea parameters that will be used to synthesize radar images. Fig. 4.7 shows the 2D Draupner spectrum obtained from data of the European Centre for Medium-Range Weather Forecasts, Reading, U.K., but rotated over an angle of $13^{\circ}$ in the western direction to have the maximum energy flux directed towards the South. The spectrum is scaled such that the significant

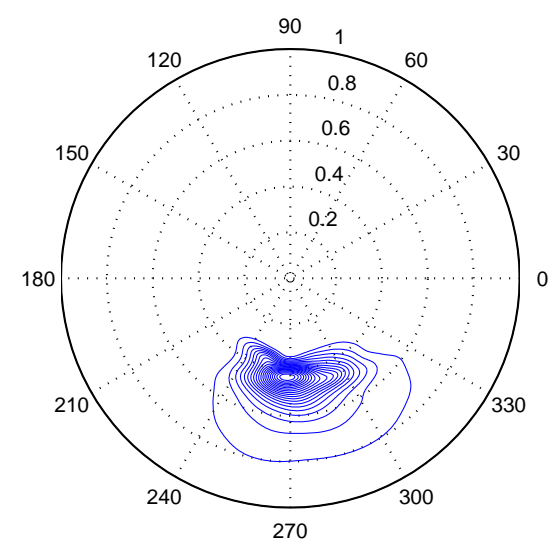

Figure 4.7: The 2D spectrum, rotated over an angle of $13^{\circ}$ to the west to have the main energy propagation to the South. 
wave height is $12 \mathrm{~m}$ and has $120^{\circ}$ spreading. The peak period is $14.45 \mathrm{~s}$ and the water depth is $70 \mathrm{~m}$. To generate the nonlinear waves based on the depicted spectrum the HAWASSI-AB third order code has been used, see van Groesen et al. 2017] for more details. From 40 ensemble seas, a sea with a maximum amplitude of $21.73 \mathrm{~m}$ is chosen. A coordinate shifting is taken such that the maximum amplitude happens near the origin where the radar will be located. The resulted sea at the time when the highest crest occurs is given in Fig. 4.8
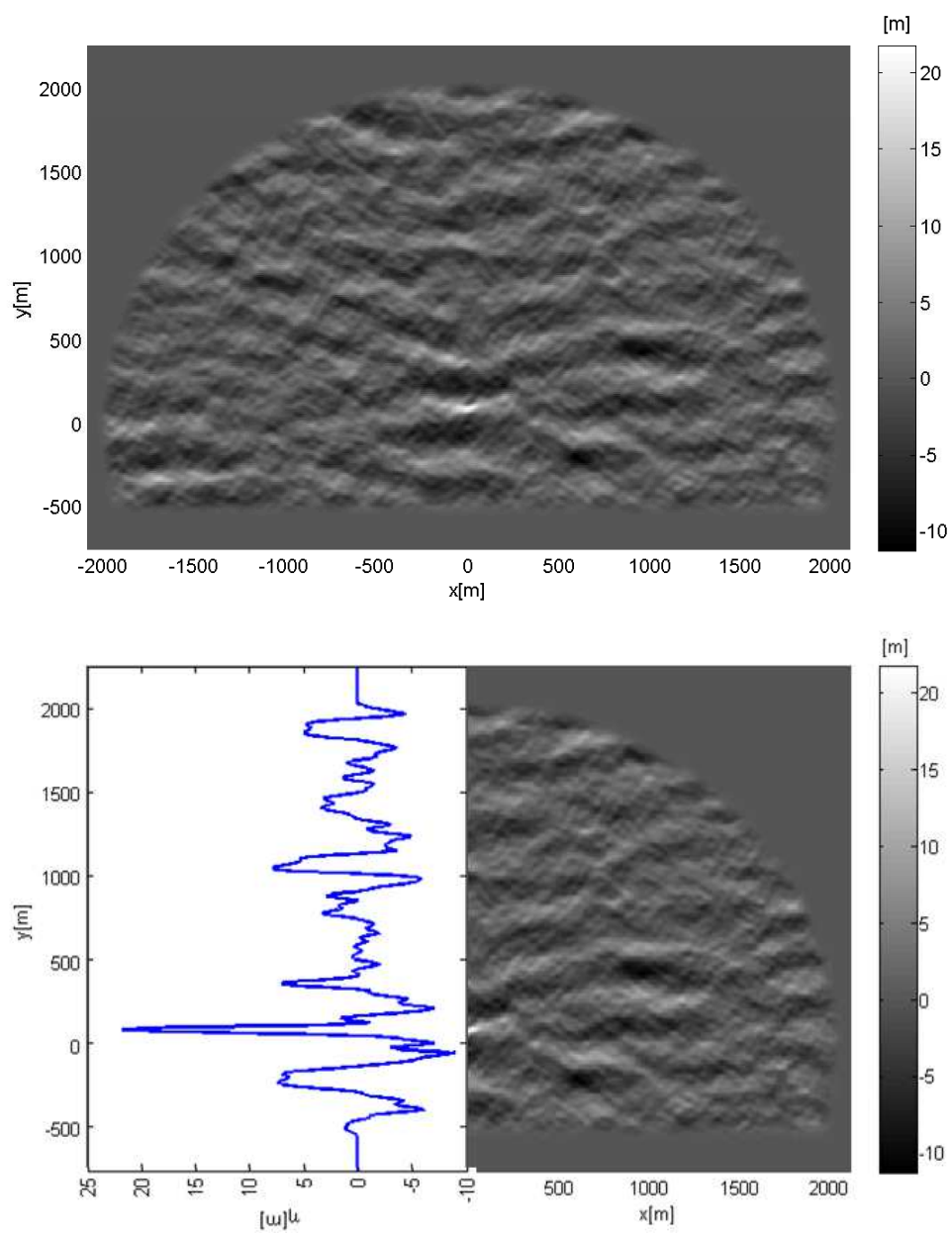

Figure 4.8: A snapshot of nonlinear Draupner waves at the time when the maximum elevation of $21.73 \mathrm{~m}$ occurs (top). The location of the freak wave is at (0,85). Part of the same density sea for $x>0$, with the left part replaced by the cross section along $x=0$ (bottom). 


\subsubsection{Dynamic averaging and evolution of synthetic images}

Shadowing is greatly determined by the dimensionless number which is the ratio between the radar height $H_{r}$ and the significant wave height $H_{s}$; the smaller this ratio, the more shadowing and the more distorted the images will be. For the radar height we take as example $H_{r}=30 \mathrm{~m}$ above the still water level, which is a reasonable value for large ships such as oil and gas tankers. This then leads to a rather small ratio of $H_{r} / H_{s}=2.5$ for Draupner seas. To obtain synthetic images for a radar
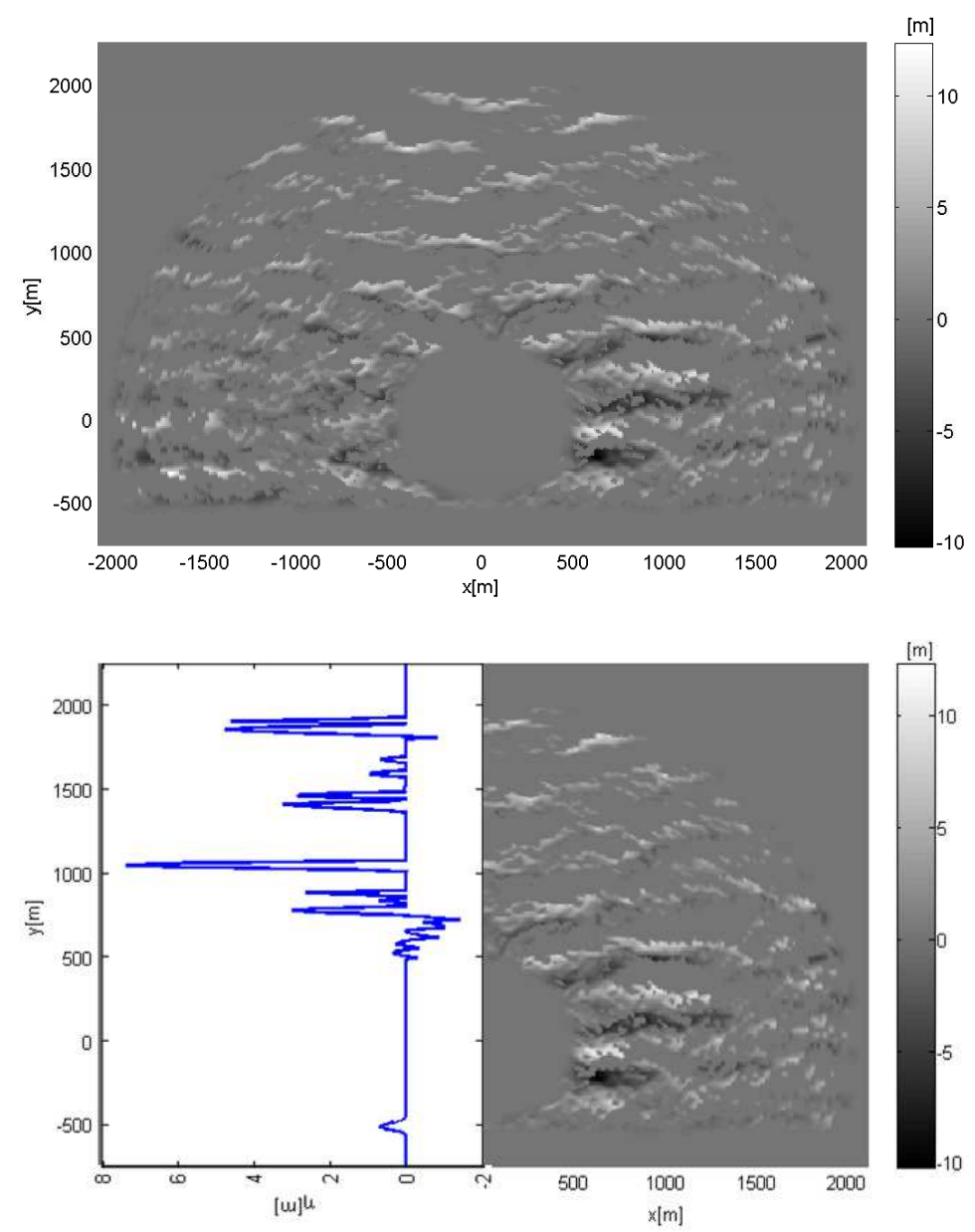

Figure 4.9: In the upper plot a shadowed image on a semicircle; zero values in the observation area $500<r<2000$ represent the shadowed area. In the lower plot part of the same density image for $x>0$, with the left part replaced by the cross section along $x=0$ showing the shadowed parts.

located at $x=(0,0)$, snapshots $\eta(\mathbf{x}, m \Delta t)$ from the given nonlinear sea are taken at discrete time differences $\Delta t=3 \mathrm{~s}$ which is approximately a quarter peak wave 
period. Then the effect of shadowing is applied to obtain distorted images $I_{m}(x)$ that will serve as synthetic radar images outside the blind zone. Fig. 4.9 illustrates the effect of shadowing. The area where the waves are not visible by the radar due to shadowing will be given the value 0 .

To improve the quality of the wave profile, two operations are performed as in Chapter 2, First dynamic averaging of 3 successive radar images is applied to improve the quality. Then the image is rescaled to have the correct significant wave height. In this paper it is assumed that the significant wave height is known in advance, although it is possible to detect the significant wave height from the radar images without external information as in Chapter 3.

One averaged scaled image is taken as initial sea state over the radar-observation domain $500<r<2000$, to start a nonlinear evolution over the length of an update, i.e. $3 \Delta t$. The evolution will start to advance the waves into the blind zone $r<500$ $\mathrm{m}$. Proceeding with a new dynamically updated image that is smoothly merged with the evolved sea state, the evolution scenario transports the waves further into the blind area, and also improves the quality in the outside region. By following Eq. 2.12, the updated image with adjusted DAES is given by

$$
\begin{aligned}
U_{0}(\mathbf{x}) & =\left(\frac{1}{6}\left(R_{0}+\varepsilon^{1}\left(R_{-1}\right)+\varepsilon^{2}\left(R_{-2}\right)\right)+\frac{1}{2} \varepsilon_{n l}^{3}\left(U_{-1}\right)\right)\left(1-\chi_{r a d}\right) \\
& +\varepsilon_{n l}^{3}\left(U_{-1}\right) \chi_{r a d}
\end{aligned}
$$

where $\varepsilon_{n l}($.$) denotes the nonlinear evolution operator which is the Analytic Boussi-$ nesq model of HAWASSI software LabMath-Indonesia, 2015.

From a practical point of view of most interest is the quality of the evolution at the radar position and in a circular area around the radar. The quality will depend on various factors, the amount of shadowing determined by the ratio $H_{r} / H_{s}$, the radius of radar observation, and the quality of the evolution operator. The use of synthetic data makes it possible to quantify the reconstruction by comparing the reconstructed sea with the design sea. As an example, the reconstructed sea and the actual sea along the cross section in the main propagation direction of the waves at $x=0 \mathrm{~m}$ is shown in Fig. 4.10. It indicates that the DAES method can reconstruct the waves

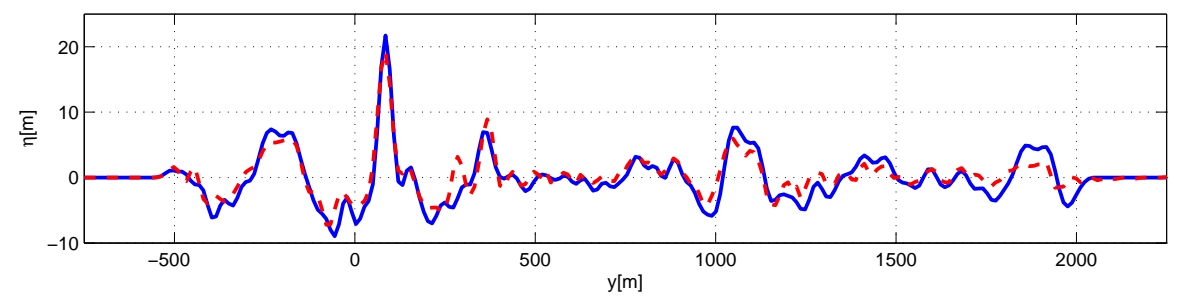

Figure 4.10: A reconstructed wave profile along $x=0$ (red-dashed line) and the actual wave elevation (blue-solid line). The extreme crest of $21.73 \mathrm{~m}$ is estimated by the reconstructed waves by a height of $19.25 \mathrm{~m}$

quite well near the radar location; further away from the radar the resemblance is 

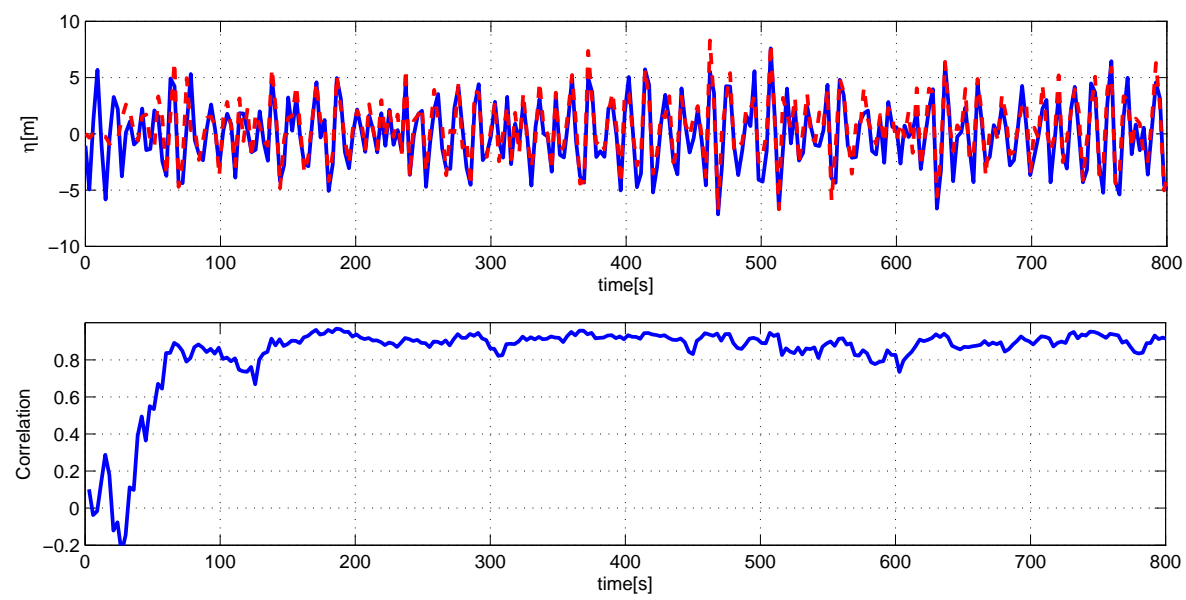

Figure 4.11: In the upper plot the time signal of the reconstructed waves (red-dashed) and the true signal (blue-solid) at the radar location. In the lower plot the correlation of the reconstructed and the true elevation in the area around the radar of radius $200 \mathrm{~m}$.

somewhat less because shadowing is more severe and fewer updates in newly entered waves have been assimilated.

The time signal at the radar location is shown in the upper plot of Fig. 4.11. At the initial stages of the process, the reconstructed waves are poor because the entrance into the blind zone of waves from outside is not yet complete near the radar. After the initiation time, the phase and amplitude of the reconstructed signal are in good agreement with the actual signal. As a quantitative result the correlation of the reconstructed and the actual sea in a radius of $200 \mathrm{~m}$ around the radar is shown in the lower plot of Fig. 4.11. The average correlation is $88 \%$.

\subsubsection{Prediction Results}

\section{Prediction Horizon}

To be able to detect the sea near the radar ahead in time, the reconstructed sea at a specific time is used as initial condition for a successive evolution. Provided the simulation is faster than real time, this can give warnings for high waves in advance. How far ahead in time the sea near the radar can be calculated depends fundamentally on the size of the observation area. Roughly speaking it is the time in which the most energy carrying waves will evolve from the outer ring to the radar. For the seas under investigation this leads to a value somewhere between $2000 / V_{g} \approx 150 s$ and $2000 / C_{p} \approx 90 s$, where $V_{g}$ and $C_{p}$ are the group and phase velocity at the peak frequency respectively.

The quality of prediction is measured as the correlation between the simulated sea and the nonlinear sea in a circular radar area of $200 \mathrm{~m}$. Averaged over 40 reconstructed wave profiles with $48 \mathrm{~s}$ time difference between two consecutive profiles, two different correlations are given as function of time in Fig. 4.12 . 


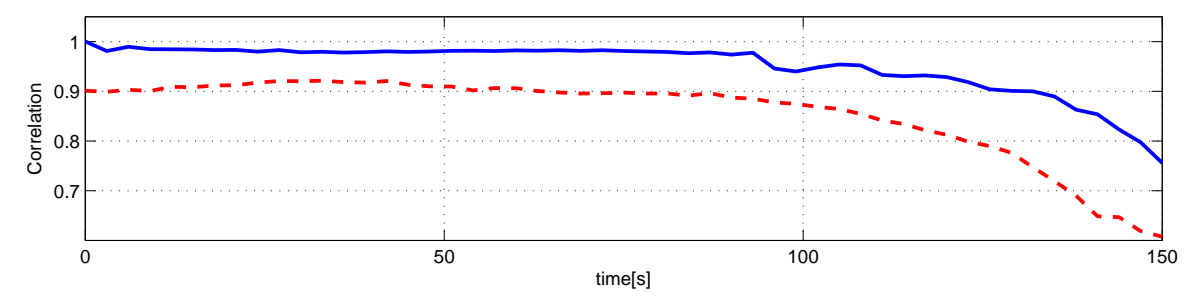

Figure 4.12: Averaged correlation in a circular area of 200m around the radar between the prediction starting with an exact initial sea state and the exact sea (blue solid), correlation between the prediction starting from a reconstructed sea state compared to the original nonlinear sea states (red dashed).

First, to check the numerical procedure and calculate the maximal prediction horizon, the solid-blue curve is the correlation between a prediction starting from a sea state of the original, unperturbed, sea and the original sea. The correlation is nearly maximal when it can be expected, i.e. until the energy carrying waves have past the radar area. After that, this correlation was observed to have a sharp decrease near $t=100 \mathrm{~s}$, indicating that this is the maximal prediction horizon, to be achieved only for perfectly reconstructed seas. The red-dotted line compares the prediction starting from a reconstructed sea state with the original sea. The result is as expected from the above: starting at the value of $90 \%$ of the reconstructed sea as initial value, the prediction remains almost constant until $100 \mathrm{~s}$ and decays slowly till a value $85 \%$ at $110 \mathrm{~s}$ and $80 \%$ at $120 \mathrm{~s}$.

\section{Prediction of a Freak-Wave}

This section discusses a possibility to detect a Freak wave from the reconstructed waves. A warning of a potential high wave a few minutes in advance will be very beneficial for a helmsman to navigate his ship in a safer way. To aim that we simulate wave predictions that a freak wave will occur half to one minute in the future. We took three reconstructed seas, at times $t=-60,-45,-30 \mathrm{~s}$ before the freak wave event, as initial profiles for wave prediction. Fig. 4.13 shows the reconstructed sea at $t=-60 \mathrm{~s}(\mathrm{a})$ and prediction results $(\mathrm{b}-\mathrm{d})$. The prediction at time $t=0 \mathrm{~s}$ (the time when the freak wave occurs) gives a maximum crest of height $16.37 \mathrm{~m}$ instead of $21.73 \mathrm{~m}$ at the position near the actual freak wave's location which can be seen in the cross section along the $y$-axis in Fig. 4.13. The blue line denotes the true wave elevation and the predicted waves are depicted in red-dashed line. The predicted time signal at the position of the freak wave is given in Fig. 4.13 . Although the prediction is not quite good the indication of the possible freak wave is already detected; a crest of 1.3 times the significant wave height is found. A better prediction is achieved by using the information at later time; maximum crest heights of $18.6 \mathrm{~m}$ and $16.78 \mathrm{~m}$ are achieved from the prediction using the reconstructed sea at times $t=-45 \mathrm{~s}$ and $t=-30 \mathrm{~s}$ respectively. It is not only the amplitudes that are better estimated, but also the location of the freak wave is well predicted. 

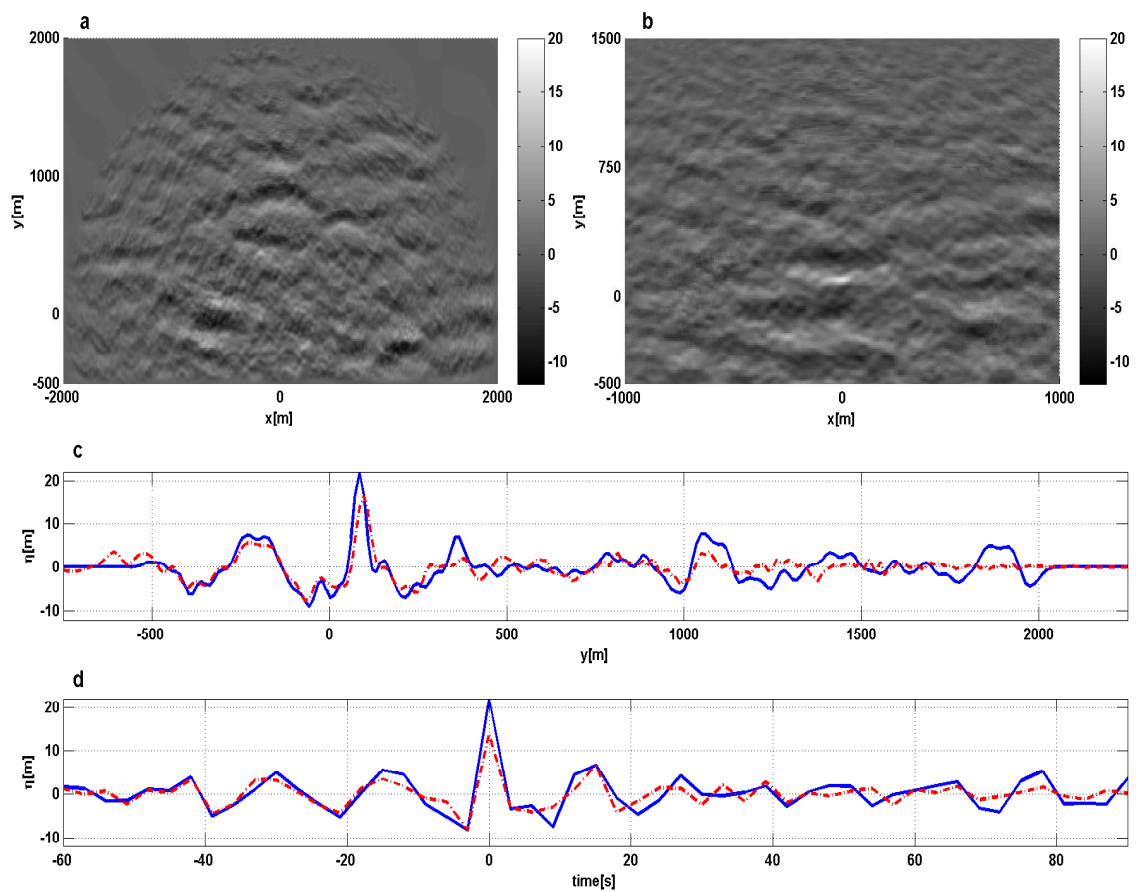

Figure 4.13: (a) The reconstructed sea at time $t=-60 \mathrm{~s}$ before the freak wave event. (b) The predicted sea at the time of the freak wave event in a smaller domain $-1000<x<1000$ and $-500<y<1500$. (c) The cross section at $x=0$ of the predicted waves (red-dashed) and the true wave elevation (blue-solid). (d) The time signal of the predicted waves (reddashed) and the true elevation (blue-solid) at the position of the freak wave. Here, $t=0 \mathrm{~s}$ denotes the time when the freak wave happens. 

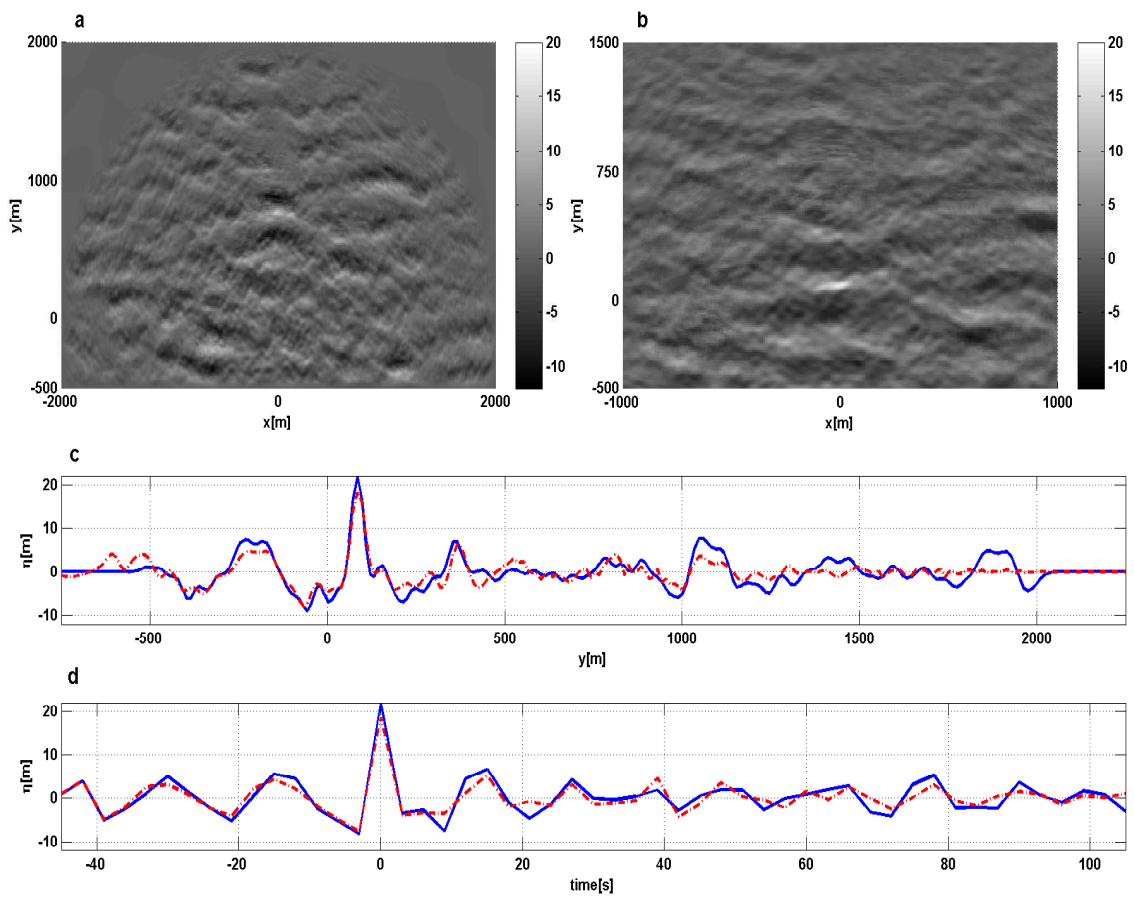

Figure 4.14: The same as in Fig. 4.13, but now for time $t=-45$ s. 

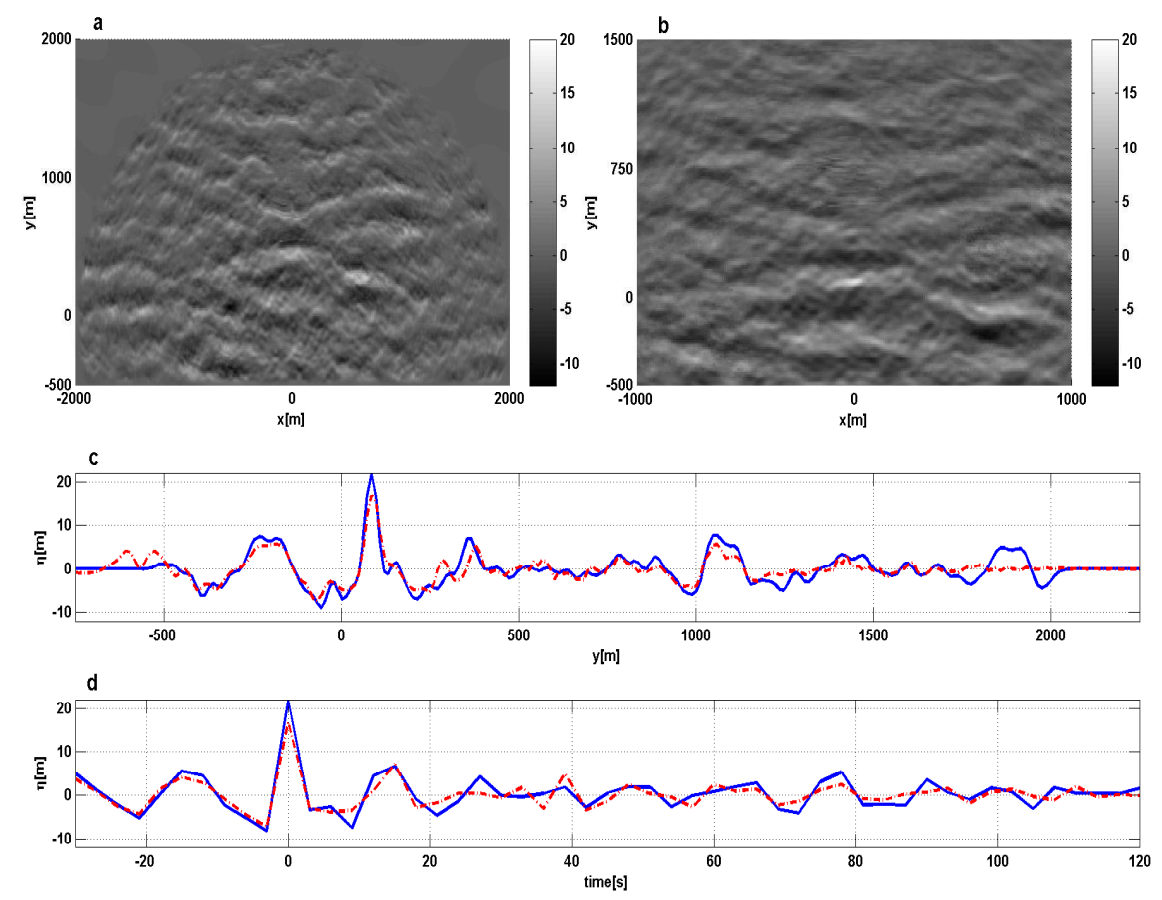

Figure 4.15: The same as in Fig. 4.13, but now for time $t=-30 \mathrm{~s}$. 


\subsubsection{Discussion}

To put the results obtained here in perspective, a comparison will be made with similar results in Chapter 2 of linear bi-modal low seas with $H_{s} \approx 3 \mathrm{~m}$ and $H_{r} / H_{s}=$ 5 and wide spreading caused by the presence of a low-amplitude swell with $T_{p}=16 \mathrm{~s}$ under an angle of 135 degrees with a wind wave system with $T_{p}=9 \mathrm{~s}$.

Compared to these seas, the reconstruction of the shadowed Draupner sea has approximately the same quality; the high correlation with the real sea and the reconstructions in Fig. 4.11 show that also for the high nonlinear Draupner seas a good reconstruction can be made.

This good reconstruction of the sea states results in a reasonably good prediction of the waves ahead of time. With the maximal prediction horizon around $120 \mathrm{~s}$, the prediction obtained for the reconstructed sea is still above $85 \%$ until $110 \mathrm{~s}$, which is slightly longer than the time for the waves to travel with the phase speed from the outer ring and less than the time to transport the energy to the radar from the outer regions.

The result for the time interval in which the freak-wave-train approaches the radar, the reconstruction identifies the waves quite well at the radar position, and also in the prediction, although the amplitude is slightly lower, some $19.25 \mathrm{~m}$ (18.44 $\mathrm{m}$ for the prediction) crest height instead of $21.73 \mathrm{~m}$. From the quality of reconstruction and prediction, we can conclude that also a single freak event, that has a life time of only $30 \mathrm{~s}$ can be predicted around $60 \mathrm{~s}$ in advance of its appearance, which seems the largest possible time interval. 



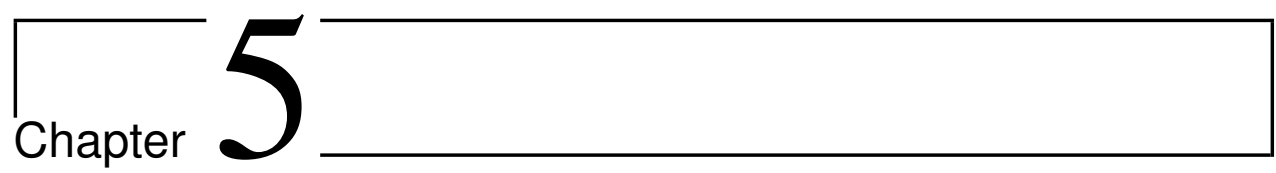

\section{Outlook}

This dissertation proposes reconstruction methods to retrieve sea surface elevations, significant wave height and sea surface current from synthetic radar images without any external calibration. Shadowing is considered as the main effect in the radar mechanism to synthesize the images. However, it is desired to test the proposed methods on the real radar data.

The wave inversion to fill in the gaps at the shadowed areas is presented in Chapter 2. The study cases consider images that are created under the condition of $H_{r} / H_{s}=5$. Although the images do not show most of the wave troughs the DAES method can improve the quality measured by the correlation with the exact sea from 0.7 to 0.95 . In Chapter 4.2 the DAES method is extended to reconstruct images which are more distorted; the nonlinearity of the waves is taken into account. The images are synthesized with lower ratio $H_{r} / H_{s}=2.5$. The nonlinear effect makes the crest higher, hence resulting in more severe shadowing. Nevertheless, the quality of the images has correlation of about 0.65 . Using the DAES method to reconstruct the images yields a better correlation of 0.88 . A prediction is carried out with correlation above $80 \%$ up to $240 \mathrm{~s}$ for the case in Chapter 2 and $120 \mathrm{~s}$ in Chapter 4.2 The possibility to predict a freak wave about $60 \mathrm{~s}$ in advance is presented in Chapter 4.2. This result is quite promising and the method can be useful for ocean engineering activities.

The method to determine the significant wave height from images based on shadowing is presented in Chapter 3 It is shown that the method is robust and can deal with various wave parameters for linear uni- and bi-modal seas. For nonlinear waves, with the same significant wave height as for linear seas, the visibility curve will be under the visibility curve derived from linear seas. The lower visibility is due to the nonlinear effect which increases crest heights (and reduces trough depths). A preliminary result to derive the significant wave height from nonlinear long-crested waves was presented in Wijaya 2016. The result gives us a confidence that the method can also deal with nonlinear short-crested seas. However, the algorithm needs to be improved to handle the computation of the nonlinear waves such that it can be useful for the real-time application. 
The DAES method is used and then adapted to estimate sea surface current from images in Chapter 4.1. The accuracy of the results are promising; it can accurately estimate current speeds ranging from $2.5 \%$ until $19.2 \%$ of the phase wave speed. The more challenging case is to take into account the speed of the vessel. The ship speed is considered as another 'current' that enters the dispersion relation. It needs further investigation to quantify the upper limit of the relative current speed that can be retrieved by the proposed method. A comparison with other methods that are based on the analysis of Fourier components (3DFFT Young et al. [1985] \& 2DFFT Alford et al. 2014]) has recently been investigated to identify the strength and the weakness of each method.

A preliminary study to apply the DAES method in the coastal area has been carried out. The research begins with a case of long-crested waves that travel from a deeper to a shallower area. A simple bathymetry is considered; constant depths $d_{0}$ and $d_{1}$ are connected with a certain slope. To deal with the varying depth the numerical code HAWASSI-AB is employed. A synthetic image, which is perturbed very much, is shown at the upper plot of Fig. 5.1. It is created from a radar located at $x=0 \mathrm{~m}$ with $500 \mathrm{~m}$ blind zone. A slope of $1 / 20$ for the bathymetry is made at $900<x<1500 \mathrm{~m}$. A reconstructed wave profile and the corresponding true elevation are depicted in the lower plot. The result has good agreement with the true elevation; wave troughs and the blind area are well recovered. The extension to short-crested waves with more complex bathymetry is a challenge for the future.
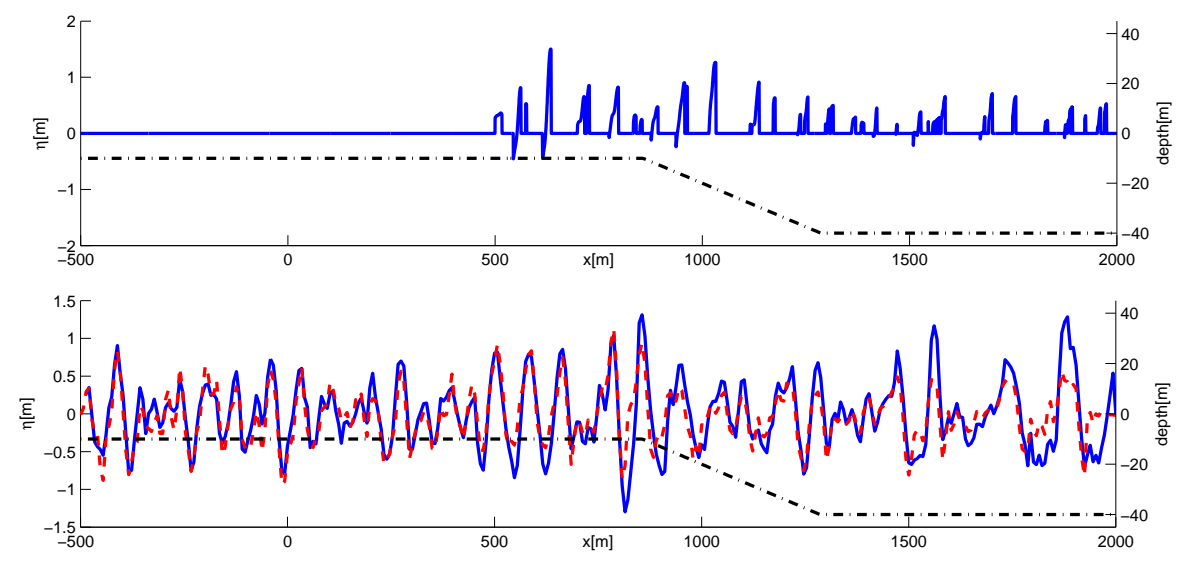

Figure 5.1: (top) A shadowed image (blue-solid) with a radar located at $x=0$ and blind zone of radius $500 \mathrm{~m}$ around the radar. (bottom) A reconstructed wave profile and the true elevation are depicted by red dashed and blue solid lines respectively. A bathymetry profile with a slope of 1/20, that connects the depth of $40 \mathrm{~m}$ and $10 \mathrm{~m}$, is given by the black dash-dotted line.

A further investigation to calculate and predict ship motion from the reconstructed sea is part of ongoing research in LabMath-Indonesia. The final aim is to develop a method that can calculate fully nonlinear wave-ship interaction from updated time signals in real time. 


\section{Bibliography}

R. Abileah and D. B. Trizna. Shallow water bathymetry with an incoherent xband radar using small (smaller) space-time image cubes. In 2010 IEEE International Geoscience and Remote Sensing Symposium, pages 4330-4333, July 2010. doi:10.1109/IGARSS.2010.5654386.

L. K. Alford, R. F. Beck, J. T. Johnson, D. Lyzenga, O. Nwogu, and A. Zundel. Design, implementation, and evaluation of a system for environmental and ship motion forecasting. In the 30th Symposium on Naval Hydrodynamics, Hobart, Australia, November 2014.

W.R. Alpers and K. Hasselmann. Spectral signal to clutter and thermal noise properties of ocean wave imaging synthetic aperture radars. J. Remote Sens., 3 (4): $423-446,1982$.

W.R. Alpers, D.B. Ross, and C.L. Rufenach. On the detectability of ocean surface waves by real and synthetic aperture radar. J. Geophys. Res., 86 (C7):6481 6498, 1981.

S. Aragh and O. Nwogu. Variation assimilating of synthetic radar data into a pseudospectral wave model. J. Coast. Res., Special issue 52:235 - 244, 2008.

S. Aragh, O. Nwogu, and D. Lyzenga. Improved estimation of ocean wave fields from marine radars using data assimilation techniques. In Proceedings of the 18th International Offshore and Polar Engineering Conference, 2008.

V. Atanassov, W. Rosenthal, and F. Ziemer. Removal of ambiguity of twodimensional power spectra obtained by processing ship radar images of ocean waves. J. Geophys. Res., C90 (1):1061 - 1067, 1985.

D. Barrick. Remote sensing of sea state by radar. In Ocean 72 - IEEE International Conference on Engineering in the Ocean Environment, pages 186-192, Sept 1972. doi 10.1109 /OCEANS.1972.1161190

D. Barrick, M. Evans, and B. Weber. Ocean surface currents mapped by radar. In Proceedings of the 1978 IEEE First Working Conference on Current Measurement, volume 1, pages 59-65, Jan 1978. doi 10.1109/CCM.1978.1158377. 
Donald E Barrick. A coastal radar system for tsunami warning. Remote Sensing of Environment, 8(4):353 - 358, 1979. ISSN 00344257. doi:http://dx.doi.org/10.1016/0034-4257(79)90034-8. URL http://www. sciencedirect.com/science/article/pii/0034425779900348.

P.S. Bell. Bathymetry derived from an analysis of $\mathrm{x}$-band marine radar images of waves. In Proceedings of the Oceanology '98 Conference, Brighton, England, Vol.3, pages 535-543, 1998.

P.S. Bell. Shallow water bathymetry derived from an analysis of X-band marine radar images of waves. Coast. Eng., 37 (3/4):513 - 527, 1999.

P.S. Bell. Mapping shallow water cosatal areas using a standard marine $\mathrm{x}$-band radar. In Proceedings of Hydro8 Conference, Liverpool, pages 535-543, 2008.

E. Blondel and P. Naaijen. Reconstruction and prediction of short-crested seas based on the application of a 3d-fft on synthetic waves: Part 2-prediction. In Proceedings of the 31st International Conference on Ocean, Offshore and Arctic Engineering, pages $55-70,2012$.

J.C.N. Borge, K. Reichert, and J. Dittmer. Use of nautical radar as a wave monitoring instrument. Coast. Eng., 37:331 - 342, 1999.

J.C.N. Borge, G.R. Rodriguez, K. Hessner, and P.I. Gonzalez. Inversion of marine radar images for surface wave analysis. J. Atmos. Ocean. Technol., 21:1291-1300, 2004.

C. Bourlier, J. Saillard, and G. Berginc. Effect of correlation between shadowing and shadowed points on the Wagner and Smith monostatic one-dimensional shadowing functions. IEEE Trans. Antennas Propag., 48:437 - 446, 2000.

R.A. Brokelman and T. Hagfors. Note of the effect of shadowing on the backscattering of waves from a random rough surface. IEEE Trans. Antennas Propag., AP-14:621 - 627, 1966.

J.R. Buckley and J. Aler. Estimation of ocean wave height from grazing incidence microwave backscatter. In Proceedings of IEEE IGARS, volume 2, pages 1015 1017, 1998a.

J.R. Buckley and J. Aler. Enhancement in the determination of ocean surface wave height from grazing incidence microwave backscatter. In Proceedings of IEEE IGARS, pages $2487-2489,1998 \mathrm{~b}$.

G.F. Clauss, S. Kosleck, and D. Testa. Critical situation of vessel operations in short crested seas forecast and decision support system. J. Offshore Mech. Arct. Eng., 134 (3):031601, 2012.

D.D. Crombie. Doppler spectrum of sea echo at 13.56 mc./s. Nature, 175:681-682, 1955. 
H. Dankert and W. Rosenthal. Ocean surface determination from X-band radar image sequence. J. Geophys. Res., 109:C04016, 2004.

A. Dzvonkovskaya, D. Figueroa, KW. Gurgel, H. Rohling, and T. Schlick. Hf radar observation of a tsunami near chile after the recent great earthquake in japan. In Radar Symposium (IRS),2011 Proceedings International, pages 125 - 130, 2011.

R. Gangeskar. Ocean current estimated from X-band radar sea surface images. IEEE Transactions on Geoscience and Remote Sensing, 40(4):783-792, 2002.

R. Gangeskar. An algorithm for estimation of wave height from shadowing in Xband radar sea surface images. IEEE Trans. Geosci. Remote Sens., 52(6):33733381, 2014.

J. Gemmrich and C. Garrett. Unexpected waves. J. Phys. Oceanogr., 38:2330 $2336,2008$.

Y. Goda. Random Seas and Design of Maritime Structures, 3rd ed. World Scientific, 2010.

Stéphan T. Grilli, Samuel Grosdidier, and Charles-Antoine Guérin. Tsunami detection by high-frequency radar beyond the continental shelf. Pure and Applied Geophysics, 173(12):3895-3934, 2016. ISSN 1420-9136. doi:10.1007/s00024-0151193-8. URL http://dx.doi.org/10.1007/s00024-015-1193-8.

K. Hessner, K. Reichert, and W. Rosenthal. Mapping of sea bottom topography in shallow seas by using a nautical radar. In 2nd International Symposium on Operationalization of Remote Sensing, Enschede, The Netherlands, August 1999.

T. Hilmer and E. Thornhill. Observations of predictive skill for real-time deterministic sea waves from the wamos ii. In OCEANS 2015 - MTS/IEEE Washington, pages $1-7$, Oct 2015. doi 10.23919 /OCEANS.2015.7404496

L. H. Holthuijsen. Waves in Oceanic and Coastal Waters. Cambridge University Press, 2007.

P. Hoogeboom and W. Rosenthal. Directional wave spectra in radar images. In International Geoscience Remote Sensing Symposium, IEEE, Munich, June 1982.

W. Huang, E. W. Gill, and Z. Zhong. Enhancement of the normalized scalar product method for surface current measurement using nautical radar. In MTS/IEEE Oceans, Hampton Roads, USA, October 2012.

P. Izquierdo, J.C.N. Borge, C.G. Soares, R.S. Gonzalez, and G. Rodriguez. Comparison of wave spectra from nautical radar images and scalar buoy data. Journal Waterway Port Coastal Ocean Engineering, 131(3):123-131, 2005.

E. Jefferys. Directional seas should be ergodic. Appl. Ocean Res., 9:186 - 191, 1987.

J.T. Johnson, R.J. Burkholder, J.V. Toporkov, D.R. Lyzenga, and W.J. Plant. A numerical study of the retrieval of sea surface height profiles from low grazing angle radar data. IEEE Trans. Geosci. Remote Sens., 47 (6):1641 - 1650, 2009. 
LabMath-Indonesia. Hawassi software, 2015. URL http://www.hawassi.labmathindonesia.org.

A. L. Latifah and E. van Groesen. Coherence and predictability of extreme events in irregular waves. Nonlinear Processes in Geophysics, 19(2):199-213, 2012.

B. Lipa, D.E. Barrick, J. Bourg, and B.B. Nyden. HF radar detection of tsunamis. Journal of Oceanography, 62 (5):705 - 716, 2006.

G. Ludeno, C. Brandini, C. Lugni, D. Arturi, A. Natale, F. Soldovieri, B. Gozzini, and F. Serafino. Remocean system for the detection of the reflected waves from the costa concordia ship wreck. IEEE Journal of Selected Topics in Applied Earth Observations and Remote Sensing, 7(7):3011-3018, July 2014. ISSN 1939-1404. doi:10.1109/JSTARS.2014.2321048.

Giovanni Ludeno, Ferdinando Reale, Fabio Dentale, Eugenio Pugliese Carratelli, Antonio Natale, Francesco Soldovieri, and Francesco Serafino. An x-band radar system for bathymetry and wave field analysis in a harbour area. Sensors, 15(1): 1691-1707, 2015. ISSN 1424-8220. doi:10.3390/s150101691. URL http://www . mdpi.com/1424-8220/15/1/1691.

B. Lund. Wind retrieval from shipborne nautical X-band radar data. IEEE Trans. Geosci. Remote Sens., 50 (10):3800 - 3811, 2012.

M.D. Miles and E.R. Funke. A comparison of methods for synthesis of directional seas. J. Offshore Mech. Arct. Eng., 111:43 - 48, 1987.

W.H. Munk and W.A. Nierenberg. High frequency radar sea return and the phillips saturation constant. Nature, 224:1285, 1969.

P. Naaijen and E. Blondel. Reconstruction and prediction of short-crested seas based on the application of a 3d-fft on synthetic waves: Part 1-reconstruction. In Proceedings of the 31st International Conference on Ocean, Offshore and Arctic Engineering, pages 43 - 53, 2012.

P. Naaijen and A.P. Wijaya. Phase resolved wave prediction from synthetic radar images. In Proceedings of the 33rd International Conference on Ocean, Offshore and Arctic Engineering OMAE, 2014.

P. Naaijen, K. Trulsen, and E. Blondel. Limits to the extent of the spatio-temporal domain for deterministic wave prediction. J. Int. Shipbuild. Prog., 61 (3-4):203$223,2014$.

W.J. Plant and G. Farquharson. Wave shadowing and modulation of microwave backscatter from the ocean. J. Geophys. Res., 117:C08010, 2012a.

W.J. Plant and G. Farquharson. Origins of features in wave number-frequency spectra of space-time images of the ocean. J. Geophys. Res, 117:C06015, 2012b.

W.J. Plant and L.M. Zurk. Dominant wave directions and significant wave heights from synthetic aperture radar imagery of the ocean. Journal Geophysical Research, 102:3473-3482, 1997. 
M. Punzo, C. Lanciano, D. Tarallo, et al. Application of x-band wave radar for coastal dynamic analysis: Case test of bagnara calabra (south tyrrhenian sea, italy). Journal of Sensors, 2016:6236925, 2016. doi:10.1155/2016/6236925.

K. Reichert and B. Lund. Ground based remote sensing as a tool to measure spatial wave field variations in coastal approaches. In 9th International Coastal Symposium, Gold Coast, Queensland, Australia, pages 1-5, 2007.

C.M. Senet, J. Seemann, and F. Ziemer. The near-surface current velocity determined from image sequences of the sea surface. IEEE Transactions on Geoscience and Remote Sensing, 39(3):492-505, 2001.

F. Serafino, C. Lugni, and F. Soldovieri. A novel strategy for the surface current determination from marine x-band radar data. IEEE Transactions on Geoscience and Remote Sensing, 7(2):231-235, 2010.

M. Skolnik. A review of radar sea echo. 1969.

A. Slunyaev, E. Pelinovsky, and C.G. Soares. Modeling freak waves from the North Sea. Appl. Ocean Res., 27:12-22, 2005.

B.G. Smith. Geometric shadowing of a random rough surface. IEEE Trans. Antennas Propag., AP-15 (5):668-671, 1967.

G.R. Valenzuela. Theories for the interaction of electromagnetic and oceanic waves-a review. Boundary-Layer Meteorology, 13(1):61-85, 1978.

E. van Groesen, A.P. Wijaya, P. Turnip, and R. Kurnia. High waves in Draupner seas and possibility for X-band radar observations. Submitted, invited paper for Journal of Ocean Engineering and Marine Energy, 2017.

R.J. Wagner. Shadowing of randomly rough surface. J. Opt. Soc. Am., 41 (1): 138-147, 1966.

Lewis B. Wetzel. Electromagnetic Scattering from the Sea at Low Grazing Angles, pages 109-171. Springer Netherlands, Dordrecht, 1990. ISBN 978-94-0090627-3. doi:10.1007/978-94-009-0627-3_3. URL http://dx.doi.org/10.1007/ 978-94-009-0627-3_3.

A. P. Wijaya, P. Naaijen, Andonowati, and E. van Groesen. Reconstruction and future prediction of the sea surface from radar observations. Ocean Engineering, 106:261-270, July 2015.

A.P. Wijaya. Towards nonlinear wave reconstruction and prediction from synthetic radar images. In Proceedings of the ASME 2016 35th International Conference on Ocean, Offshore and Arctic Engineering, 2016.

A.P. Wijaya. Dynamic averaging method to detect sea surface current from radar images. Accepted for publication in Proceedings of the ASME 2017 36th International Conference on Ocean, Offshore and Arctic Engineering, Trondheim, Norway, 2017. 
A.P. Wijaya and E. van Groesen. Significant wave height retrieval from synthetic radar images. In Proceedings of the 11th International Conference on Hydrodynamics, number 44, 2014. ISBN 978-981-09-2175-0.

A.P. Wijaya and E. van Groesen. Determination of the significant wave height from shadowing in synthetic radar images. Ocean Eng., 114:204-215, 2016.

G. Wu. Direct Simulation and Deterministic Prediction of Large-Scale Non-linear Ocean Wave Field. PhD Thesis, Massachusetts Institute of Technology, 2004.

I.R. Young, W. Rosenthal, and F. Ziemer. A three dimensional analysis of marine radar images for the determination of ocean wave directionality and surface currents. J. Geophys. Res., 90:1049-1059, 1985.

F. Ziemer and W. Rosenthal. Measurement of the directional wave spectra by ship radar. In IAPSO Symposium PS-11 IUGG XVIII, General Assembly, Hamburg, 1983.

F. Ziemer and W. Rosenthal. On the transfer function of a shipborne radar for imaging ocean waves. In Proceedings IGARSs'87 Symposium, pages 1559 - 1564, 1987. 


\section{Acknowledgments}

The research in this dissertation has been carried out in the Applied Analysis (AA) group, Department of Applied Mathematics, University of Twente (UT) and in LabMath-Indonesia (LMI). This research is motivated by some challenges in the Industrial Research Project entitled "Prediction of waves induced motions and forces in ship, offshore and dredging operations (Promised)", funded by the Dutch Ministry of Economical Affairs, Agentschap NL and co-funded by Delft University of Technology, University of Twente, Maritime Research Institute Netherlands, Ocean Waves GMBH, Allseas, Heerema Marine Contractors and IHC Merwede.

I have been very fortunate for the support of my supervisor, colleagues, friends, and family whom I would like to acknowledge. First of all, I would like to express my sincere gratitude to my supervisor Prof. Brenny van Groesen for giving me the opportunity to do a very interesting $\mathrm{PhD}$ and post-doc research. I learn a lot from his teaching about mathematics and his insightful guidance for me in writing a paper. Thank you also for your enthusiastic supervision and continuous support. I would also like to thank Dr. Andonowati for providing me a very nice work place in LabMath-Indonesia. I extent my gratitude to Prof. Stephan van Gils, the chair of group AA, for the opportunity to work in his group and also his willingness to be one of my graduation committee.

I would like to thank to Prof. Arthur Veldman, Prof. Arnold Heemink, Prof. Bayu Jayawardhana, Dr. Gebrant van Vledder, and Dr. Mashury Wahab for their willingness to be my committee members and to Prof. Peter Apers and Prof. Marc Uetz as the chairperson and the secretary of my graduation committee.

I would like to thank Prof. Rene Huijsmans for allowing me to work as a guest researcher in his group and Peter Naaijen for his excellent supervision during my research at TU Delft. Also to Tyson Hilmer for a nice discussion during the Promised project. I got an engineering point of view from them. I express my sincere thank to the secretary of the department of Applied Mathematics UT: Marielle SlotboomPlekenpol and Linda Wychgel for helping me to arrange all the administrative things. Thanks to my former colleagues in UT and TU Delft: Anastasia, Nida, Ruddy, Wenny, Wisnu, Abrari, Zilko, Tao, and Bong Jun. I indebted many thanks to the people during my stay in the Netherlands: Marwan Wirianto and his wife Meily 
Otrina for their warmth welcome, Kenny for sharing his room and a very nice discussion about everything, and Tante Sofieyati Hardjosumarto for her favors when I stay in Enschede.

I have to mention my LMI colleagues and the former for a fruitful friendship and discussion: Liam, Didit, Mourice, Andy Schauff, Meirita, Hafiizh, Nunu, Marc, Januar, Alif, Peri, Law, Lia, Natasha, Riam, Fanny, Adjie, David, Marcel and others. I indebted many thanks to Mira Melanie and Dian Astuti for helping me about administrative processes in LMI. I give my highest appreciation to Inez Huang who design the cover of my dissertation, thank you so much. I would also like to thank all the lectures in the Department of Mathematics, Unpar, especially to Dr. Fery Jaya Permana and Dr. Dharma Lesmono for giving me the opportunity to teach in their department. I thank to Liem Chin, Joko, Stefy, Rocky, Regina, Harry, and Mega for a very warm friendship that keep me sane during some stressful time.

Finally, I am grateful to my parents: Lioe Gunawan and Thoeng Lindawati, to my sister Rani Puspa Wijaya and her husband Leonard Setiawan and her son Carlo. A very special thank to Uncle Afung and Uncle Simchan (and their families) for their favors. 


\section{About the author}

Andreas Parama Wijaya was born on the $4^{\text {th }}$ of December 1986 in Bandar Lampung, Indonesia. He received his Bachelor of Science degree from the Mathematics department of Parahyangan Catholic University (Unpar), Indonesia in January 2009 on a subject of numerical method for solving a wave equation with a pulse source. Since August 2008, he has been teaching on various mathematics subjects for Engineering Faculty and Science Faculty of Unpar. In August 2009, he continued his study on Master program in the Mathematics department of Institut Teknologi Bandung (ITB), Indonesia. He finished his Master's thesis in July 2011 on a subject of integral equation for an inverse wave problem. In February 2012, he worked as an internship student in LabMath-Indonesia.

In June 2012, he started his Ph.D research in the Department of Applied Mathematics, University of Twente. He executed his research partly at LabMath-Indonesia and TU Delft. In July 2017, he finished his doctoral studies. The results of his research is presented in this dissertation. Starting from 1 March 2017, he works as a post-doctoral researcher at University of Twente and LabMath-Indonesia. His research is about the use of HAWASSI code for nonlinear wave reconstruction from radar images. 


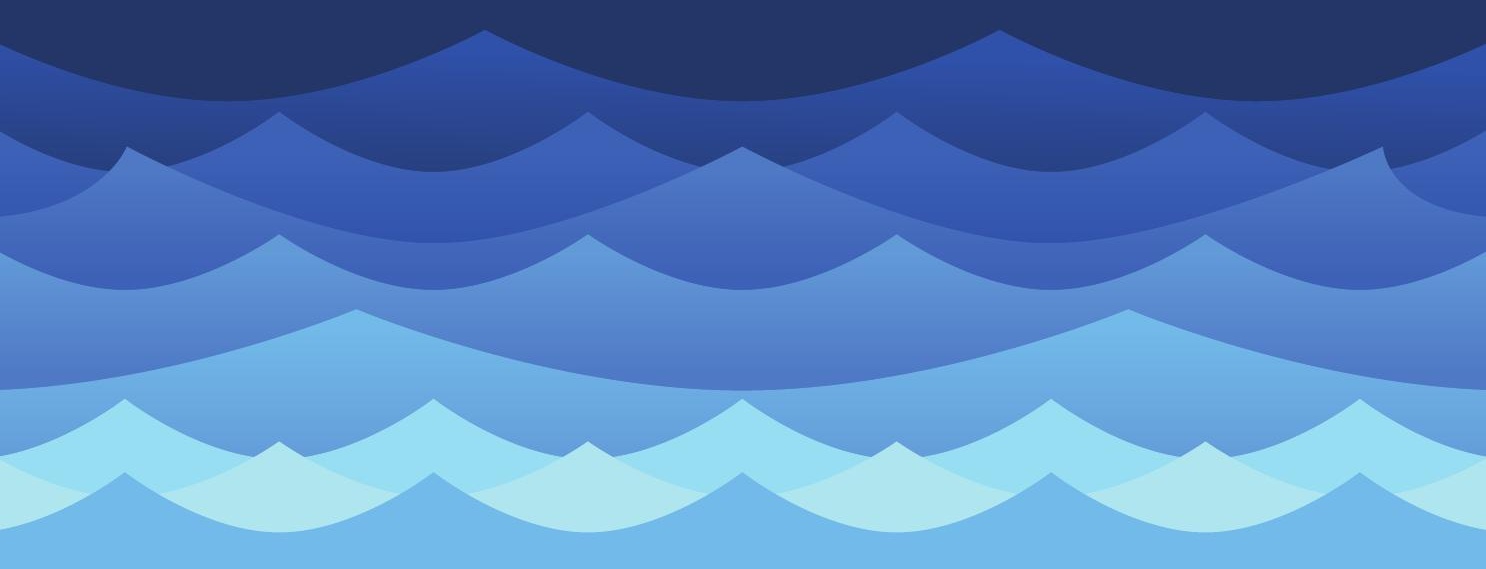

